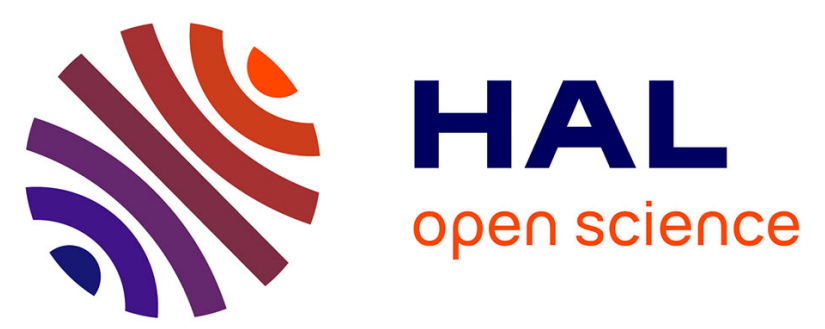

\title{
Oedenburg. Une agglomération d'époque romaine sur le Rhin supérieur : fouilles françaises, allemandes et suisses à Biesheim-Kunheim (Haut-Rhin)
}

Michel Reddé, Hans Ulrich Nuber, Stefanie Jacomet, Jörg Schibler, Caty Schucany, Peter-Andrew Schwarz, Gabriele Seitz, Francesca Ginella, Martine Joly, Suzanne Plouin, et al.

\section{To cite this version:}

Michel Reddé, Hans Ulrich Nuber, Stefanie Jacomet, Jörg Schibler, Caty Schucany, et al.. Oedenburg. Une agglomération d'époque romaine sur le Rhin supérieur: fouilles françaises, allemandes et suisses à Biesheim-Kunheim (Haut-Rhin). Gallia - Archéologie de la France antique, 2005, 62, pp.215-277. 10.3406/galia.2005.3070 . hal-01911507

\section{HAL Id: hal-01911507 https://hal.science/hal-01911507}

Submitted on 29 Jan 2020

HAL is a multi-disciplinary open access archive for the deposit and dissemination of scientific research documents, whether they are published or not. The documents may come from teaching and research institutions in France or abroad, or from public or private research centers.
L'archive ouverte pluridisciplinaire HAL, est destinée au dépôt et à la diffusion de documents scientifiques de niveau recherche, publiés ou non, émanant des établissements d'enseignement et de recherche français ou étrangers, des laboratoires publics ou privés.

\section{(ㅇ)(1) $\$$}

Distributed under a Creative Commons Attribution - NonCommercial - NoDerivatives 44.0 


\title{
OEDENBURG
}

\section{UNE AGGLOMÉRATION D'ÉPOQUE ROMAINE SUR LE RHIN SUPÉRIEUR}

\author{
Fouilles françaises, allemandes et suisses sur les communes \\ de Biesheim et Kunheim (Haut-Rhin)
}

\section{Coordination de Michel REDDÉ*}

Hans Ulrich NuBER, Stefanie JACOMET, Jörg SCHIBLER, Caty SCHUCANY, Peter-Andrew SCHWARZ, Gabriele SEITZ avec la collaboration de Francesca GINELLA, Martine JOLY, Suzanne PlOUIN, Heidemarie HÜster Plogmann, Christophe Petit, Laurent POPOVITCH, Angela SCHLUMBAUM, Patricia VANDORPE, Bénédicte VIROUlet, Lucia WiCK, Ijean-Jacques WOLF, Bastien GISSINGER, Vincent OLLIVE, Julien PELLISSIER

Mots-clés. Oedenburg, Rauraques, armée romaine, limes, camp, agglomération secondaire, temples, paléoenvironnement, archéozoologie, paléobotanique, Valentinien, palais, forteresse, église.

Résumé. L'établissement gallo-romain d'Oedenburg à Biesheim et Kunheim (Haut-Rhin, France) est fouillé depuis 1998 par une équipe internationale (École pratique des hautes études, Paris ; université de Fribourg-en-Brisgau, Allemagne; université de Bâle, Suisse). Le site, au bord du Rhin actuel, en face de l'oppidum celtique de Breisach, était, dans l'Antiquité, installé dans un paysage sensiblement différent, traversé alors par différents chenaux du fleuve. Dans l'état actuel des connaissances, les lieux semblent occupés, au début du règne de Tibère, par un camp militaire romain dépendant de la zone de commandement de Vindonissa, au nord du territoire rauraque. Un élablissement civil se développe dans le même temps, faisant peut-être suite à une occupation indigène sporadique. Dès le milieu du $\Gamma^{p r}$ s. apr.J.-C. sont perceptibles des traces de parcellaire dans le plan d'urbanisme, mais l'agglomération (qu'on identifie de manière hypothétique avec Argentovaria) n'est pas organisée comme un cheflieu de cité; on n’y rencontre en effet ni centre public ni trame viaire régulière, mais un complexe religieux composé de plusieurs temples de type indigène. Après le départ des militaires, vers 69-70, l'établissement civil se développe de manière autonome. I'importantes études archéozoologiques et paléobotaniques, favorisées par des conditions de conservation exceptionnelles, mettent en évidence les processus de romanisation et l'introduction de pratiques alimentaires nouvelles.

On ignore pour l'instant si le site fut remilitarisé dans la seconde moitié du IIT s., au moment où le Rhin redevint frontière.

Les constructions de l'Antiquité tardive se concentrent alors sur les zones qui échappent aux inondations. C'est seulement sous Valentinien qu'on voit apparaître sur la rive gauche un palais fortifié, qui répond sans doute à la forteresse de Breisach. Le site n'est pas abandonné après les invasions du début du ves. Plusieurs églises successives et une nécropole s'installent ensuite sur les ruines de l'ancienne fortification.

Key-words. Oedenburg, Rauraci, Roman army, limes, military camp, vicus, sanctuary, palaeoenvironment, archaeozoology; archaeobotany, Valentinian, palatium, fortress, church.

Abstract. The Gallo-Roman settlement of Oedenburg (communities of Biesheim and Kunheim, Dép. Haut-Rhin, France) is being excavated by an international team (École pratique des hautes études, Paris, France; University of Freiburg im Brisgau, Germany

\footnotetext{
* Voir les adresses des auteurs p. 270.
} 
and the University of Basel, Switzerland). Situated along the river Rhine and opposite the oppidum of Breisach, the settlement was embedded in quite a different landscape with many different arms of the Rhine running through it. In the present state of our knowledge, the site seems to be occupied at the beginning of the reign of Tiberius, by a military camp attached to the commanding area of Vindonissa, located in the northern part of the territory settled by the indigenous Rauraci. A civilian settlement developed at the same time, perhaps succeeding to a sporadic indigenous occupation. From the mid of the $I^{\text {st }}$ century AD) traces of plots appear in the urban plan, but the town (which can hypothetically be identified as Argentovaria) is not organised like a capital of civitas. There is no evidence for a town center nor for a rectangular road network, but only a sanctuary with several Gallo-Roman temples. After the withdrawal of the military unit around 69/70 AI) the civilian settlement developed on its own. Archaeozoological and archaeobolanical analysis, favoured by exceptional good preservation, make evident the process of Romanization and the adoption of new eating habits.

At the present time, it is not known if the settlement was again occupied by the Roman army in the second half of the $3^{\text {rd }}$ century AD when the Rhine again became a frontier: Construction works of the Late Antiquity then concentrate on areas which are protected from floodings. During the reign of Valentinian a fortified palace (palatium) was built, without doubt an equivalent to the fortress of Breisach on the left bank of the Rhine. The site was not abandoned after the German invasions in the early $5^{\text {th }}$ century. On the ruins of the old palace several successive churches were installed and a cemetery was drawn up.

Translation : Peter-Andrew SCHWARZ and Susan L.̈̈THI

Schlagwörter. Oedenburg, Rauriker, römische Armee, Limes, Militärlager, Vicus, Tempel, antike Umwell, Archäozoologie, Archäobotanik, Valentinian, Palatium, Befestigung, Kirche.

Zusammenfassung. Die römische Fundstelle Oedenburg (Biesheim-Kunheim, Haut-Rhin, France) wird seit 1998 von einem internationalen Team ausgegraben (École pratique des hautes études, Paris, Universität Freiburg im Breisgau, Deutschland, Universität Basel, Schweiz). Gegenüber dem keltischen Oppidum von Breisach, nahe am heutigen Rhein gelegen, war die Siedlung in einer deutlich anderen Landschaft angelegt worden, die damals von verschiedenen Flussarmen durchzogen war: Nach dem bisherigen Stand der Lrforschung scheint der Ort zu Beginn der tiberischen Zeit von einem Militürlager besetzt gewesen zu sein, das zum Kommandobereich von Vindonissa im Nordteil des Territoriums der Rauriker gehörte. Zur gleichen Zeit entwickelte sich eine Zivilsiedlung, die vielleicht auf eine sporadische einheimische Belegung folgte. Ab der Mitte des 1. Jahrhunderts n. Chr. werden im Überbauungsmuster Spuren einer Parzellierung erkennbar, doch entspricht die Organisation der Siedlung (die man hypothetisch mit Argentovaria gleichsetzt) nicht jener eines (ivitas-Vorortes; man kann weder ein öffentliches Zentrum noch ein orthogonales Straßenraster erkennen, sondern einzig einen Kultbezirk mit mehreren gallo-römischen Umgangstempeln. Nach Abzug des Militärs um 69/70 verlief die Entwicklung der Zivilsiedlung autonom. Umfangreiche, durch außergewöhnliche Erhaltungsbedingungen begünstigte archäozoologische und archäobotanische Untersuchungen zeigen den Romanisierungsprozess und die Einführung neuer Essgewohnheilen auf. Zurzeit ist unbekannt, ob man den Ort in der zweiten Hälfte des 3. Jahrhunderts wieder militärisch beselzt hatte, als der Rhein erneut zur Grenzzone wurde. Die spätantiken Anlagen konzentrieren sich auf die überschwemmungssicheren Zonen. Erst in valentinianischer Zeit ist ein befestigtes Palatium nachzuweisen, das zweifellos eine linksrheinische Entsprechung zur Befestigung von Breisach darstellt. Der Platz wurde nach den Invasionen zu Beginn des 5. Jahrhunderts nicht verlassen. In den Ruinen der vormaligen Festung werden mehrere aufeinander folgende Kirchen und ein Friedhof eingerichtet.

Übersetzung : Caty SCHUCANY

L'agglomération gallo-romaine d'Oedenburg est située sur la rive occidentale du Rhin, communes de Biesheim et de Kunheim (Haut-Rhin), en face de la ville allemande de Breisach (fig. 1). Le toponyme antique n'est pas connu avec certitude. L'hypothèse d'une identification avec Argentovaria, mentionné comme $\pi 0 \lambda i \varsigma$ des Rauraques par Ptolémée (II, 9, 9), puis signalé sur l'Itinéraire Antonin (Cünt\% éd., 1990, p. 353,3 ) et la Table de Peutinger, est de plus en plus fréquemment avancée (Fellmann, 1995), mais n'a pas encore été formellement prouvée par des témoignages épigraphiques.

Oedenburg, déjà cité en 1551 par Beatus Rhenanus, apparaît sur la carte de J. D. Specklin en 1576 (Plouin dir., 2001). Les premières découvertes archéologiques remontent à la fin du XVII ${ }^{c}$ s. (Schöpflin, 1849), et des fouilles furent entreprises en 1824 par P. de Golbéry (Golbéry, 1828). Mais c'est surtout à partir de la construction du canal de dérivation du Rhin, en 1868, que l'on entend parler du site (Biellmann,
1996). Avaient alors été mises au jour une série d'amphores, diverses monnaies du Haut-Empire, de la céramique et une dizaine de pieux de bois. Quelques années plus tard, A. Cestre, ancien conducteur des travaux du Rhin, relate la découverte de vestiges à l'occasion du creusement d'un fossé parallèle au canal de dérivation (Biellmann, 1996). Il publie en outre un plan topographique qui a le mérite de montrer la position d'Oedenburg en bordure d'un fleuve non encore endigué (Cestre, 1884). Un autre relevé, jusqu'ici inédit, montre la forteresse d'Altkirch et la limite de l'inondation mémorable survenue en 1852 (voir fig. 44).

Différents sondages ont été effectués depuis les années 1960, d'abord par C. Bonnet et M. Jehl, puis par P. Carl et E. Kern. Ces sondages n'ont été que sommairement publiés ou ne sont connus que par les informations de Gallia. Tout récemment, la Carte archéologique du Haut-Rhin en a fait une recension abondante, qui nous dispense ici d'une longue 


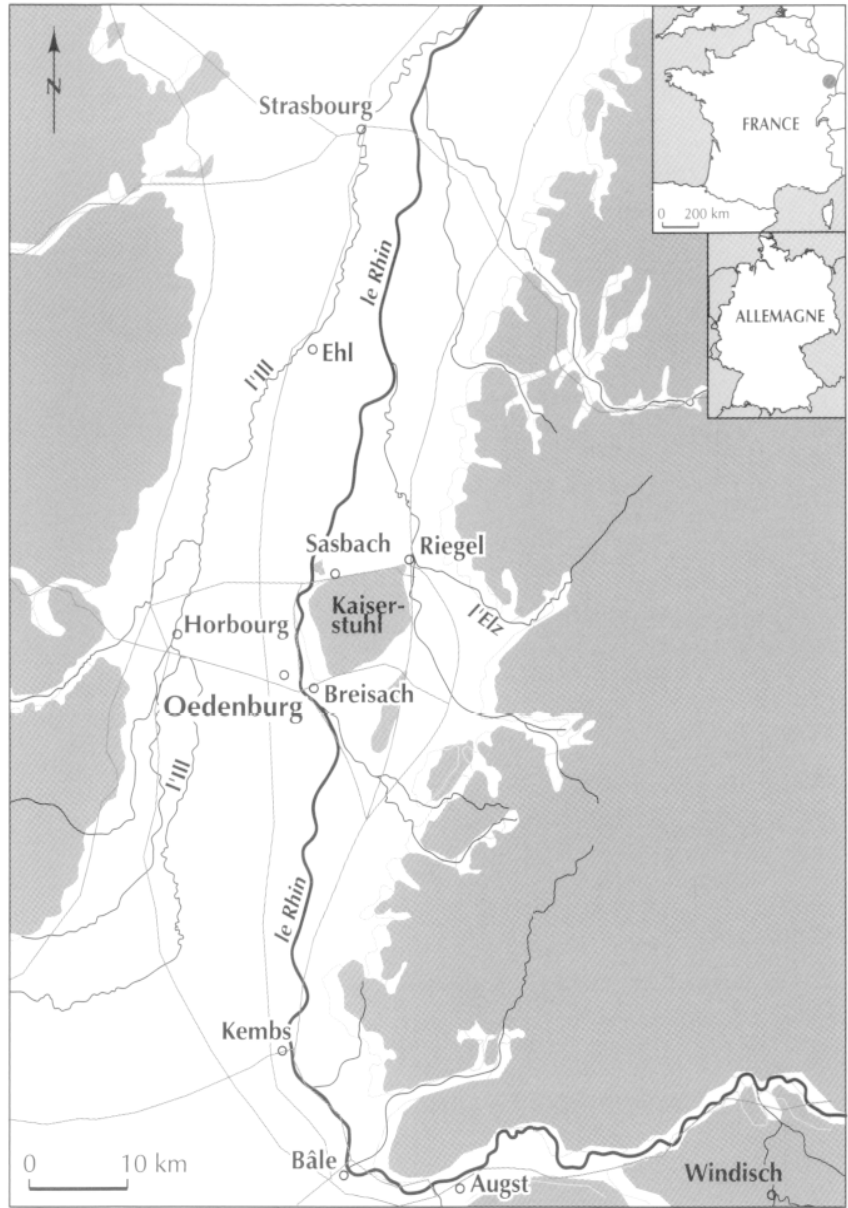

Fig. 1 - La situation d'Oedenburg dans la région du Rhin supérieur (dessin M. Reddé, EPHE, d'après un fond de carte de l'université de Fribourg-en-Brisgau).

description (Zehner, 1998). Le site a livré un grand nombre de tuiles estampillées au timbre de différents corps de troupe (tabl. I) (Biellmann, 1997).

Il existe trois couvertures aériennes obliques du site, l'une réalisée par $\mathrm{O}$. Braasch, l'autre par $\mathrm{R}$. Goguey, la troisième par $\mathrm{J}$-J. Wolf. Le musée gallo-romain de Biesheim abrite aujourd'hui de très riches collections, issues principalement des nombreuses prospections pédestres effectuées au fil du temps par l'association "Archéologie et histoire de Biesheim », sous la direction de P. Biellmann, ainsi que d'une petite partie des sondages des années 1970-1980.

Depuis 1998 a été engagée une importante opération pluriannuelle de fouilles sur cette agglomération dont on savait jusqu'alors très peu de choses, sauf que sa stratigraphie couvrait toute l'époque romaine jusqu'à l'Antiquité tardive comprise. L.initiative en a été prise conjointement par H. L. Nuber (université de Freiburg im Breisgau) et M. Reddé (École pratique des hautes études), avec l'appui du Service départemental archéologique du Haut-Rhin et des collectivités locales concernées. L'université de Bâle a rejoint le projet dès 1999, de sorte qu'aujourd'hui cette entreprise archéolngique concerne les trois pays riverains du bassin du Rhin supérieur, chacun ayant
Tabl. I - Les estampilles militaires sur tuiles (état 1997, d'après P. Biellmann).

\begin{tabular}{|l|c|}
\hline Corps de troupe & Nombre d'estampilles \\
\hline Legio I Martia & 53 \\
\hline Legio IV Macedonica & 1 \\
\hline Legio VIII Augusta & 52 \\
\hline Legio XI Claudia & 13 \\
\hline Legio XIV Gemina & 1 \\
\hline Legio XXI Rapax & 80 \\
\hline Cohors XXVI vol. C. R. & 2 \\
\hline Non identifiées & 3 \\
\hline Total & $\mathbf{2 0 5}$ \\
\hline
\end{tabular}

en charge une problématique scientifique précise. Un premier rapport intermédiaire faisant le point des acquis en 2001 a été publié dans Germania (Nuber, Reddé et al., 2002). L'objectif du présent article est de présenter de manière synthétique quelques-unes des réflexions que suscite cette recherche collective ${ }^{1}$, à l'issue de la campagne 2003 . Le programme triennal en cours doit se poursuivre jusqu'en 2005.

M. R.

1. L'ensemble de l'opération est coordonné par M. Reddé (École pratique des hautes études, Paris), titulaire de l'autorisation de fouilles, mais chaque équipe dispose de ses crédits propres. Nous souhaitons à ce propos remercier les institutions partenaires qui assurent le financement du projet: pour la France, le SIVOM Hard-Nord, maître d'ouvrage, la Direction régionale des affaires culturelles d'Alsace, le Conseil général du Haut-Rhin, les communes de Biesheim et de Kunheim ; pour l'Allemagne, M. le Recteur de l'université de Fribourg, le ministère de la Wissenschaft, Forschung und Kunst BadenWürttemberg à Stuttgart, et la Deutsche Forschungsgemeinschaft à Bonn ; pour la Suisse le Fonds zur Förderung der Lehre und Forschung de l'université de Bâle, le Max-Gelder-Fonds à Bâle et la FreiwilligAkademische Gesellschaft (FAG) à Bâle.

Les objectifs scientifiques ont été déterminés d'un accord commun et sont définis de la manière suivante:

- fouille des camps julio-claudiens et de l'agglomération civile (niveaux précoces) : équipe française, sous la direction de M. Reddé. J.J. Wolf (Service départemental archéologique du Haut-Rhin), J. Pellissier (Antea) et B. Gissinger dirigent les différents chantiers ;

- fouille de l'agglomération civile et des temples : équipe suisse (université de Bâle), sous la direction de F. Sicgmund (2000) puis de C. Schucany et de P.A. Schwar\% (2002-2003) ;

- fouille des niveaux et monuments tardifs de Westergass et d'Altkirch : équipe allemande (université de Freiburg im Breisgau) sous la direction de H. U. Nuber et G. Seitz;

- géoarchéologie : C. Petit, arec la collaboration de V. Ollive (université de Dijon) ;

- archéobotanique : équipe suisse (université de Bâle) sous la direction de S. Jacomet, assistée de P. Vandorpe, A. Schlumbaum et L. Wick; - archéozoologie : équipe suisse (unirersité de Bâle) sous la direction de J. Schibler, assisté de F. Ginella, H. Hüster Plogmann ;

- céramologie : M. Joly (université de Paris-IV) et B. Viroulet (Service départemental archéologique du Haut-Rhin) ; C. Schucany (université de Bâle).

Le présent article est le fruit d'un travail collcctif, qui cngagc l'ensemble des signataires. Les passages relatifs à l'Antiquité tardive ont 


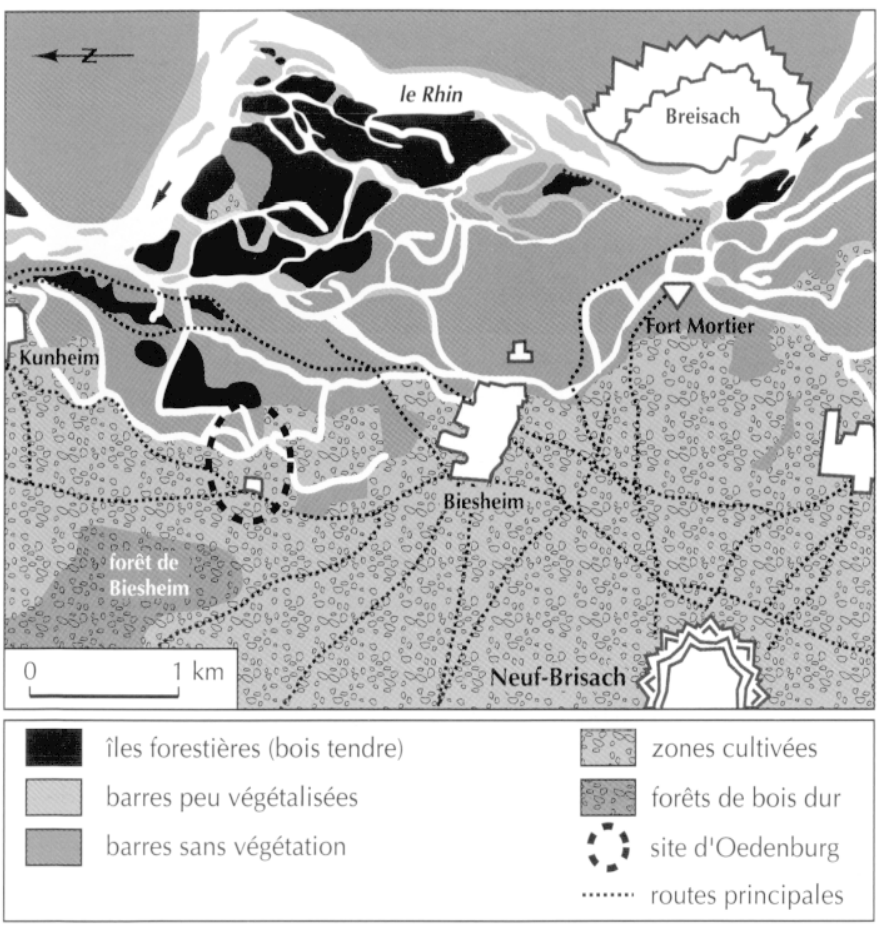

Fig. 2 - La position du site archéologique d'Oedenburg en bordure du Rhin, avant la canalisation du fleuve (dessin C. Petit et V. Ollive, univ. de Dijon).

\section{LE SITE}

\section{I.A PIAINE D'AISACE FT DE BADF.}

La plaine d'Alsace et de Bade correspond à un fossé d'effondrement séparant les Vosges et la Forêt-Noire, dans lequel se sont accumulés depuis l'Oligocène (25 Ma) plus de $1300 \mathrm{~m}$ de sédiments. Au cours du Plio-Quaternaire (2 Ma), plus de $200 \mathrm{~m}$ de galets et graviers rhénans se sont mis en place (Blanalt et al., 1974; Théobald et al., 1978). La plaine est parcourue par deux rivières principales :

- sur la bordure occidentale, l'Ill, qui draine le Jura septentrional et les collines du Sundgau, présente un régime hydrologique de type pluvio-nival avec des crues au cours de l'automne et de l'hiver (Vogt, 1988) ;

été rédigés par $\mathrm{H}$. U. Nuber et G. Seitz; ceux qui portent sur le secteur des temples sont dus à P. Schwarz et C. Schucany. Le chapitre consacré à l'étude archéozoologique a été préparé par F. Ginella, avec la collaboration de H. Hüster Plogmann, sous la direction de J. Schibler; l'étude archéobotanique est due à P. Vandorpe, avec la collaboration de A. Schlumbaum et L. Wick, sous la direction de S. Jacomet. Les chapitres consacrés à la géoarchéologie ont été écrits par C. Petit, avec la collaboration de V. Ollive. Le reste du texte a été rédigé principalement par M. Reddé, avec la collaboration de J-J. Wolf et B. Viroulet, B. Gissinger, J. Pellissier, M. Joly, L.. Popovitch (université de Dijon). La coordination de l'ensemble de l'article a été assurée par M. Reddé. De nombreux autres collaborateurs, que nous remercions très vivement, participent à cette entreprise collective. Pour des raisons de place, les études céramologiques ne figurent pas ici : elles feront l'objet d'une publication séparée. Mais leurs résultats ont naturellement été utilisés dans cet article.
- à l'est, le Rhin débouche dans la plaine à Bâle, après avoir drainé le massif alpin et la plaine molassique suisse, et longe le massif de la Forêt-Noire et du Kaiserstuhl. Il présente un fort débit et une importante charge solide : de grandes barres graveleuses caractéristiques d'un système fluviatile en tresses sont bien visibles en plan (fig. 2) (Bristow, Best, 1993). Sa grande variabilité hydrographique résulte du déplacement des barres (Church, 1978; Thorne et al., 1993). Son régime hydrologique montre de grands contrastes : des crues violentes et destructrices comme en 1852 (Conrad, 1999) ou bien des étiages parfois très marqués, comme en 1304 (Martin, 1989). À la latitude de Colmar, la plaine d'Alsace présente des zones humides (Ried) et d'anciens chenaux holocènes du Rhin et de l'Ill, fréquemment inondés; ces zones humides encadrent la région plus sèche de la Hardt, terrasse fluviatile graveleuse occupée par une forêt xérothermique (Carbeiner, 1969 ; Sell et al., 1998).

Le site d'Oedenburg s'étend sur plus de $1 \mathrm{~km}$, de la terrasse alluviale à l'ouest jusque dans la basse plaine du Rhin parcourue par de nombreux chenaux dont certains sont encore actifs aujourd'hui, malgré la canalisation du fleuve effectuée au XIX ${ }^{\mathrm{e}} \mathrm{s}$.

\section{GÉOMORPHOLOGIE DU SITE D'OEDENBURG}

L'analyse géomorphologique conduite sur l'ensemble du site archéologique associe à un relevé général de la microtopographie un redressement des principales photographies aériennes, ainsi qu'un géoréférencement de l'ensemble du mobilier archéologique recueilli en prospection.

\section{LES MÉTHODES}

Les relevés microtopographiques ont été réalisés à l'aide d'un théodolite laser avec carnet électronique d'acquisition permettant un géoréférencement, dans le système de coordonnées Lambert II étendu, de plus de 6200 points suivant une maille rectangulaire régulière $(20 \mathrm{~m}$ suivant la direction nordsud et $10 \mathrm{~m}$ en est-ouest) en raison de l'axe d'allongement des principaux paléochenaux. L'interpolation a été réalisée par la méthode du krigeage à l'aide du logiciel de modélisation numérique Surfer 8.0.

Le redressement des principales photographies aériennes obliques a été réalisé avec le logiciel Airphoto ; les documents ont été sélectionnés en fonction de leur intérêt géoarchéologique (lisibilité des paléochenaux et des structures archéologiques), mais également en fonction de la possibilité de les recaler sur un plan cadastral. Ainsi, les zones humides identifient les paléochenaux du Rhin ou des creusements anthropiques (fosses, fossés, etc.) à remplissage fin. Les zones plus sèches correspondent à des structures graveleuses : soit des barres de chenaux, soit des structures anthropiques (voies, bâtiments, etc.).

L'ensemble du mobilier céramique et métallique, les tuiles et les monnaies issues des prospections aux lieux-dits Altkirch, Rheinacker, Ried, Unterfeld et Westergass ont été cartographiés sur fond cadastral puis géoréférencés à l'aide du logiciel Didger 2. Ainsi, près de 2000 monnaies datées par règne d'émission ont pu être cartographiées. 


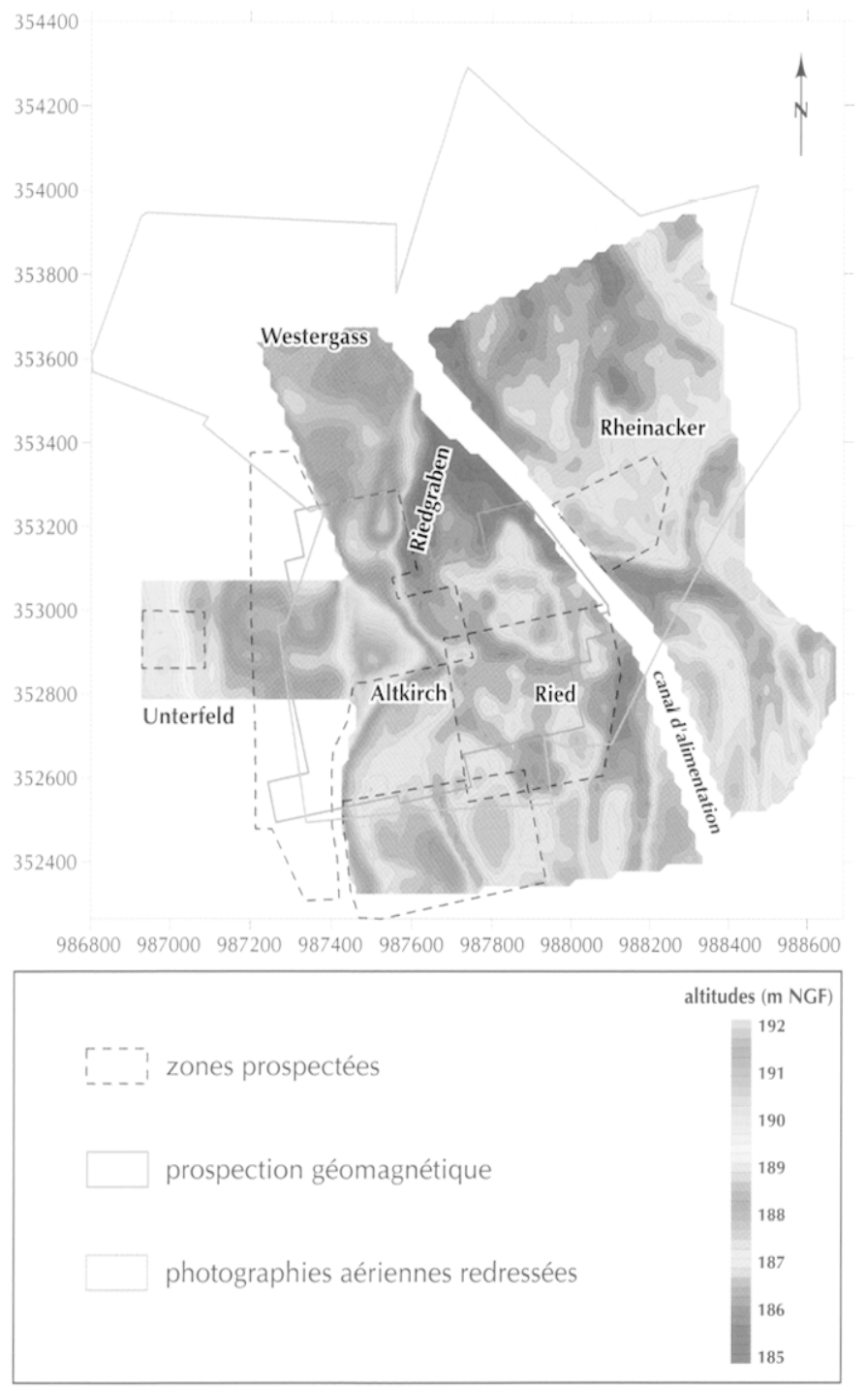

Fig. 3 - Restitution du système hydrologique d'Oedenburg (DAO C. Petit et V. Ollive, univ. de Dijon).

\section{LES RÉSULTATS GÉOMORPHOLOGIQUES}

La confrontation de ces différentes approches spatiales permet de reconstituer le paysage alluvial actuel et d'appréhender l'évolution de son occupation archéologique. Entre $185,4 \mathrm{~m}$ et $192 \mathrm{~m}$ NGF, deux principales zones géomorphologiques différentes peuvent être identifiées (fig. 3) :

- une zone occidentale haute et sèche (altitude généralement supérieure à $189 \mathrm{~m}$ ) située aux lieux-dits Altkirch, Unterfeld et Westergass. Elle correspond au sommet de la terrasse graveleuse weichsélienne rhénane empruntée par la D 468. Au nord de Westergass, des paléochenaux (segments sombres) se superposent, sur les couvertures aériennes, à des barres longitudinales et transversales (zones claires), mais ils semblent tous antérieurs à l'époque antique ;

- la zone orientale s'étend aux lieux-dits Ried et Rheinacker et correspond à la plaine inondable holocène du Rhin, où plusieurs unités morphologiques peuvent être identifiées. Au sud, plusieurs paléochenaux délimitent des barres longitudinales de taille variable; au nord, deux grandes barres sablograveleuses, de part et d'autre du canal actuel, sont clairement séparées par de larges chenaux méandrisants; la plus orientale est occupée par le camp julio-claudien dont les différentes générations de fossés sont bien visibles sur les photographies aériennes (fig. 9). Le chenal le plus occidental, longeant le rebord de terrasse, porte le nom de Riedgraben.

L'étude de la géomorphologie alluviale de ce secteur de la plaine du Rhin prouve que le site antique d'Oedenburg est en partie implanté sur la terrasse weichsélienne du Rhin, mais que la plus grande partie du site se trouve dans une zone basse, largement parcourue par de nombreux chenaux latéraux, actifs pendant tout l'Holocène.

\section{LES DÉPLACEMENTS DE L'OCCUPATION HUMAINE}

L'évolution de la répartition spatiale du mobilier monétaire recueilli en prospection montre que l'occupation du site d'Oedenburg s'est déplacée au cours des quatre premiers siècles de notre ère, bien que le site n'ait pas été prospecté de façon homogène. Quatre grandes phases d'occupation peuvent être identifiées (fig. 4).

La phase $\mathbf{A}$ correspond à la première moitié du I ${ }^{\mathrm{er}} \mathrm{s}$ apr. J.-C. Les monnaies émises sous la République et sous les empereurs julio-claudiens sont présentes dans tous les secteurs du site. Il faut noter cependant une hétérogénéité dans leur répartition : les monnaies républicaines et augusto-tibériennes sont principalement concentrées au lieu-dit Rheinacker.

La phase $\mathbf{B}$ correspond à la seconde moitié du $I^{\mathrm{er}}$ s. et au $\mathrm{II}^{\mathrm{e}} \mathrm{s}$. La répartition des monnaies est très différente de la période précédente : elles sont bien représentées à Altkirch, à Unterfeld, le long de la route départementale, ainsi que dans le sud du Ried, alors que le lieu-dit Rheinacker est déserté.

La phase C, qui correspond au III $^{\mathrm{e}}$ s. au sens large (de 192 à 312), présente un nombre assez limité de monnaies sur l'ensemble du site d'Oedenburg.

La phase D correspond au Ive s., de 312 à 407/411. Un nombre très important de monnaies, plus d'un millier, a été retrouvé principalement sur la terrasse, aux lieux-dits Unterfeld, Altkirch et Westergass, mais l'occupation sporadique de la basse plaine alluviale persiste.

Cette analyse permet de proposer une chronologie monétaire de l'occupation antique du site ; celle-ri montre un abandon précoce du secteur du camp (Rheinacker) dès l'époque flavienne, un développement du vicus, à la fois sur la butte d'Altkirch et dans les zones basses, du $\mathrm{I}^{\mathrm{er}} \mathrm{s}$. à la fin du $\mathrm{II}^{\mathrm{e}}$ s., et un repli sur la terrasse au cours du IV $\mathrm{e}$. On peut naturellement s'interroger sur les raisons qui ont conduit les hommes à délaisser les zones basses, inondables, dans le courant du $\mathrm{I}^{\mathrm{e}} \mathrm{s}$. : avons-nous affaire à une remontée importante de la nappe phréatique, ou le phénomène s'explique-t-il, au contraire, par d'autres causes, plus directement liées à la situation historique?

Dans le secteur du Ried, en contrebas de la butte d'Altkirch, l'analyse de la répartition précise de l'ensemble du mobilier antique par rapport aux structures archéologiques identifiées sur les cartes géomagnétiques et drapées sur le modèle 

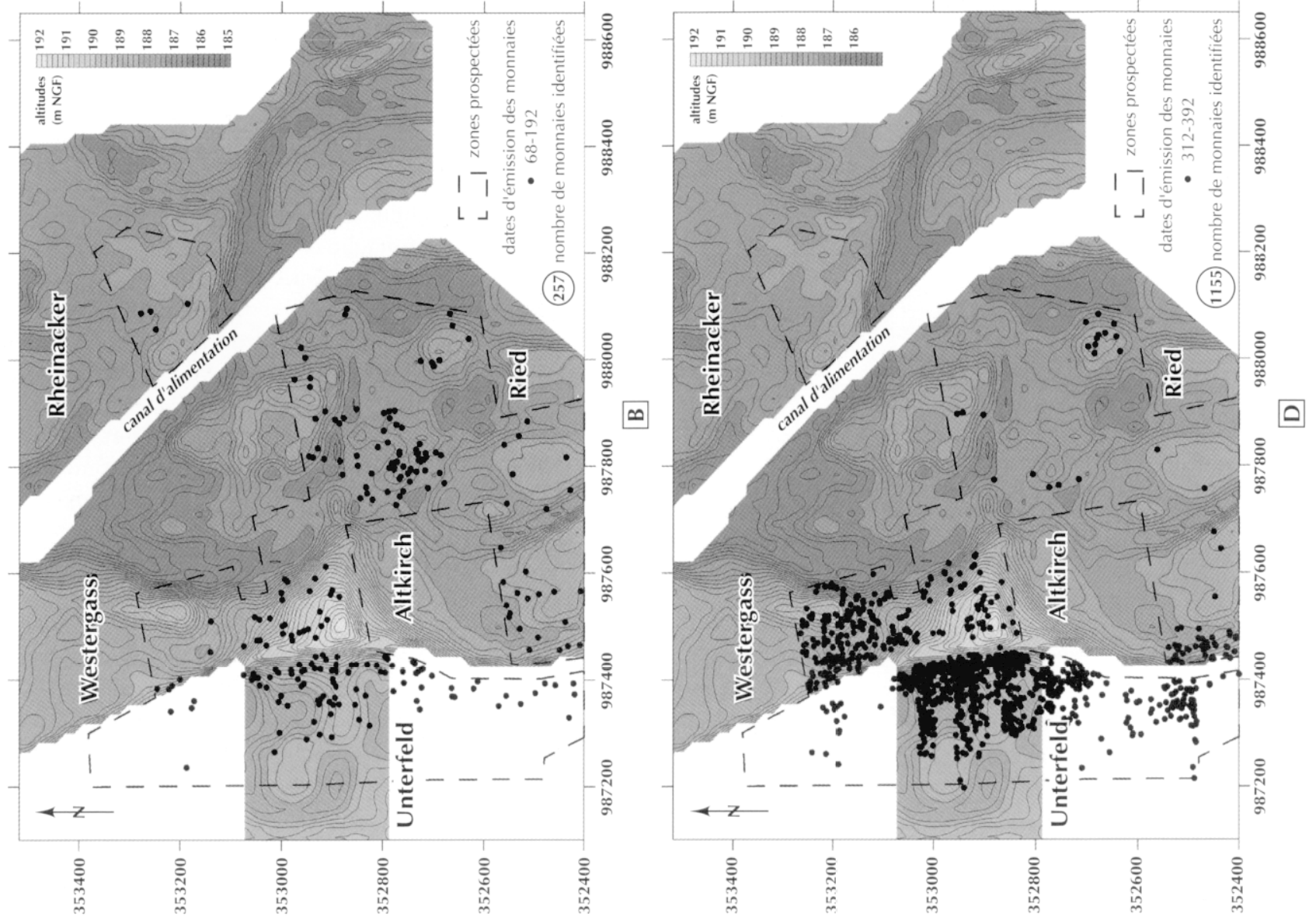

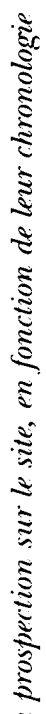
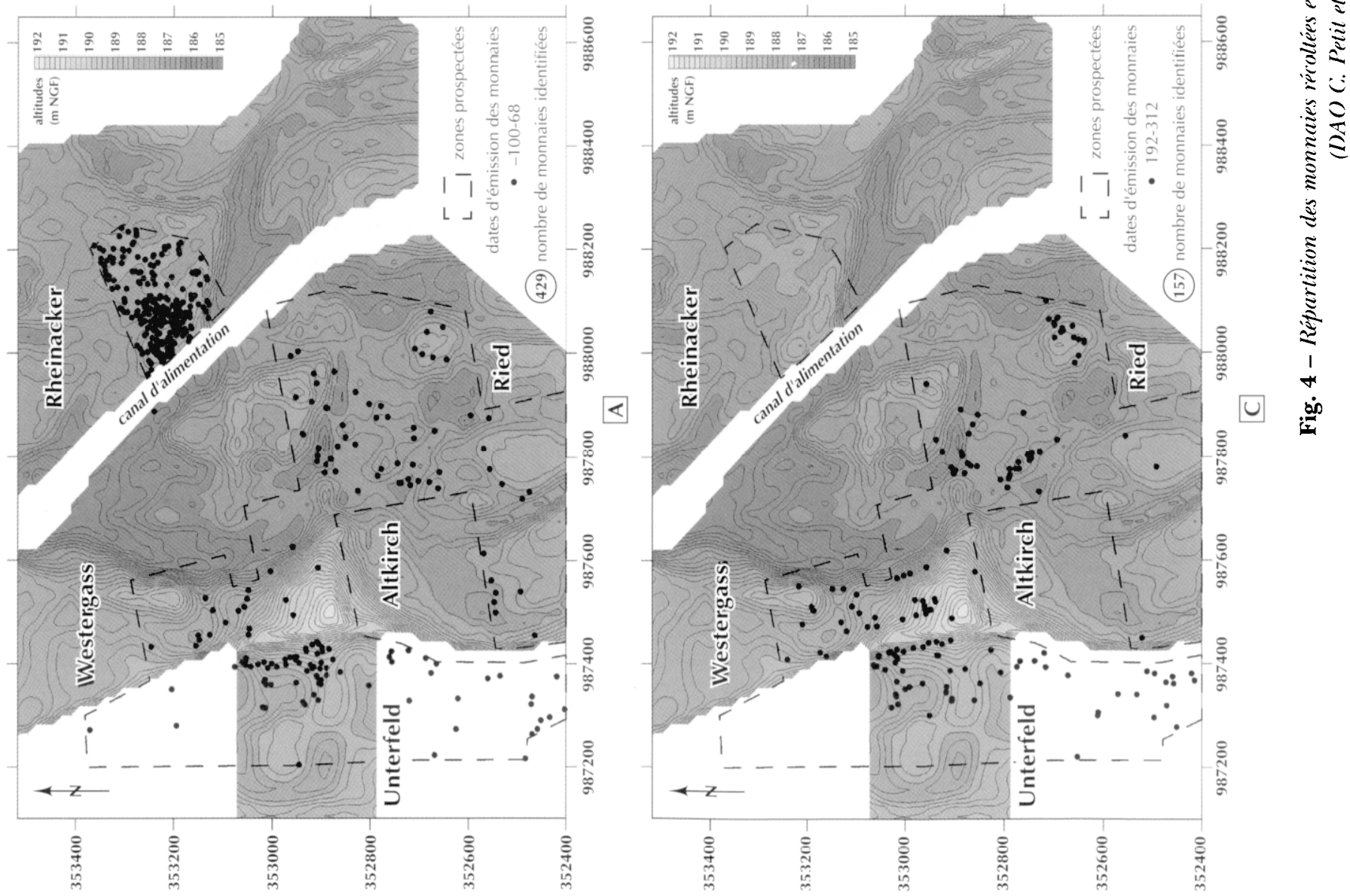
numérique de terrain montre que les fortes densités de mobilier s'organisent le long des axes de circulation ou à proximité des zones densément construites.

C. P. et V. O.

\section{LA TOPOGRAPHIE DE L'OCCUPATION HUMAINE}

Le site antique d'Oedenburg est installé à un carrefour de voies : la route sud-nord, venant d'Italie, empruntait la rive gauche du Rhin pour se diriger vers la mer du Nord; signalons aussi la route ouest-est, venant de Metz et de Horbourg, qui passait par le Kaiserstuhl (fig. 1).

\section{CARACTÈRES GÉNÉRAUX ET PRINCIPAUX ENSEMBLES D'OEDENBURG}

L'organisation générale du site est désormais assez bien connue, grâce aux reconnaissances aériennes et à la prospection géomagnétique effectuée sur une cinquantaine d'hectares par la firme allemande Posselt und Zickgraf $\mathrm{GmbH}^{2}$ (fig. 5). Plusieurs ensembles, qui ne sont pas nécessairement contemporains, doivent être distingués (fig. 6).

\section{ENSEMBLE 1}

Au nord-est, en bordure du canal d'alimentation, apparaît un ensemble de camps julio-claudiens installés sur une barre graveleuse (voir infra, p. 225). L'angle d'un autre complexe militaire précoce, non précisément daté, apparaît plus au nord (hors fig. 6), près du Giessen (bras actif le plus occidental du Rhin), sur certaines photographies aériennes de O. Braasch.

\section{ENSEMBLE 2}

À la sortie sud-ouest des camps, l'image géophysique révèle une série de grands bâtiments. Leur forte "signature " magnétique indique probablement des bâtiments en dur (basalte du Kaiserstuhl, tuiles), ce qui suppose une chronologie sensiblement postérieure à celle du complexe militaire julio-claudien, construit en terre et en bois. De fait, les fouilles menées en bordure de la voie nord-est - sud-ouest qui sort des camps ont révélé des constructions sous-jacentes en bois, invisibles dans la prospection géophysique, et de nombreuses fosses qui présentent une "signature " magnétique ponctuelle, en raison du matériel céramique qu'elles contiennent. Ces ensembles sont julio-claudiens ou flaviens (voir infra, p. 231-232).

Le bâtiment 1, qui s'inscrit dans un carré d'environ $50 \mathrm{~m} \mathrm{x}$ $50 \mathrm{~m}$, est centré sur une cour interne bordée d'un quadriportique qui donne accès à diverses pièces. L'entrée principale, au nord-est, semble précédée par une grande halle. À titre d'hypothèse de travail, et sans avoir la place de discuter ici cette interprétation, nous proposerons, eu égard aux caractéristiques du

2. La prospection géophysique a été financéc par les trois équipes partenaires. plan, d'y reconnaître une mansio ${ }^{3}$. Le bâtiment 2 occupe une superficie voisine $(50 \mathrm{~m} \times 50 \mathrm{~m}$ environ). Son plan général, la présence d'une enceinte englobant des espaces séparés, de tailles et de formes diverses, sa " signature " magnétique très forte font penser à un ensemble thermal avec ses hypocaustes et ses bassins. Il est moins facile de déterminer la nature de l'ensemble 3, accolé au bâtiment 1 . Ses absides, au nord-est, sa cour, au sud-ouest, pourraient plaider pour un second complexe thermal, bien que d'autres hypothèses soient envisageables. Au sud, près du canal moderne, apparaissent clairement deux bâtiments : 4 a été fouillé et doit sans doute être interprété comme un magasin (voir infra, p. 235) ; plus au sud, 5 est un balnéaire de petite taille, partiellement exploré et datable du $\mathrm{III}^{\mathrm{e}} \mathrm{s}$. (voir infra, p. 235). Peut-être doit-il être mis en relation avec un complexe au sein duquel figure un mithraeum $\mathbf{6}$, fouillé dans les années 1970 (Pétry, Kern, 1978) et une construction non identifiée 7 , entourée par un vaste mur de clôture.

Le long de la voie nord-est - sud-ouest qui sort du camp, et que les fouilles récentes datent du deuxième quart du $\mathrm{I}^{\mathrm{er}} \mathrm{s}$. apr. J.-C. (voir infra, p. 234), apparaît une série de fosses et de structures perpendiculaires à la chaussée. On reconnaît notamment un petit ensemble thermal 8, non exploré. Les sondages de l'université de Bâle (2000 et 2002) ont mis au jour, à l'ouest de la chaussée, des zones d'ateliers métallurgiques, avec une occupation qui ne descend pas au-delà du début du $\mathrm{II}^{\mathrm{C}} \mathrm{s}$. apr.J.-C.

L'analyse de la topographie montre que tout ce secteur est installé au sommet d'une barre graveleuse; il est séparé du reste de l'agglomération, à l'ouest et au nord-ouest, par une vaste zone basse, où la prospection géophysique ne révèle guère de structures (plage grise quasi uniforme), et au sud par un chenal. Une grande voie est-ouest relie cet ensemble à la butte d'Altkirch d'un côté, au Rhin de l'autre côté. Les sondages effectués sur cette chaussée montrent que l'installation n'est pas antérieure à l'extrême fin du I ${ }^{\mathrm{er}}$ s. apr. J.-C.

\section{ENSEMBLE 3}

Une deuxième zone très densément occupée apparaît à l'ouest, sur la butte d'Altkirch et le long de la route moderne. Il est clair que l'habitat a été volontairement installé dans un secteur non inondable. L'agglomération s'étire en longueur sur la partie la plus haute de la terrasse, avec des parcelles en lanière perpendiculaires aux axes de circulation, ce que l'image géomagnétique révèle de manière particulièrement claire vers le sud. Aucune fouille n'a été effectuée dans cette zone, mais les prospections de surface, très riches, ont montré une occupation continue depuis le $\mathrm{I}^{\mathrm{er}} \mathrm{s}$. apr. J.-C. jusqu'au début du $\mathrm{v}^{\mathrm{c}} \mathrm{s}$. Vers l'ouest, la densité des vestiges construits en dur, avec une forte "signature " magnétique, disparaît assez rapidement. On observe toutefois la présence de plusieurs voies; une grande rue est-ouest, qui provient des parages de la butte d'Altkirch, avait autrefois été baptisée, un peu hâtivement, "decumanus ». Elle conduit, semble-t-il, en direction du site gallo-romain de Horbourg, dans la banlieue nord-est de Colmar. Cette rue, bien

3. Voir, parmi d'autres, des exemples très comparables à Chelmsford, Godmanchcstcr, Wanborough (Burnham, Wacher, 1990, fig. 4) et I.ydney Park (Wheeler, Wheeler, 1932, pl. 52). 


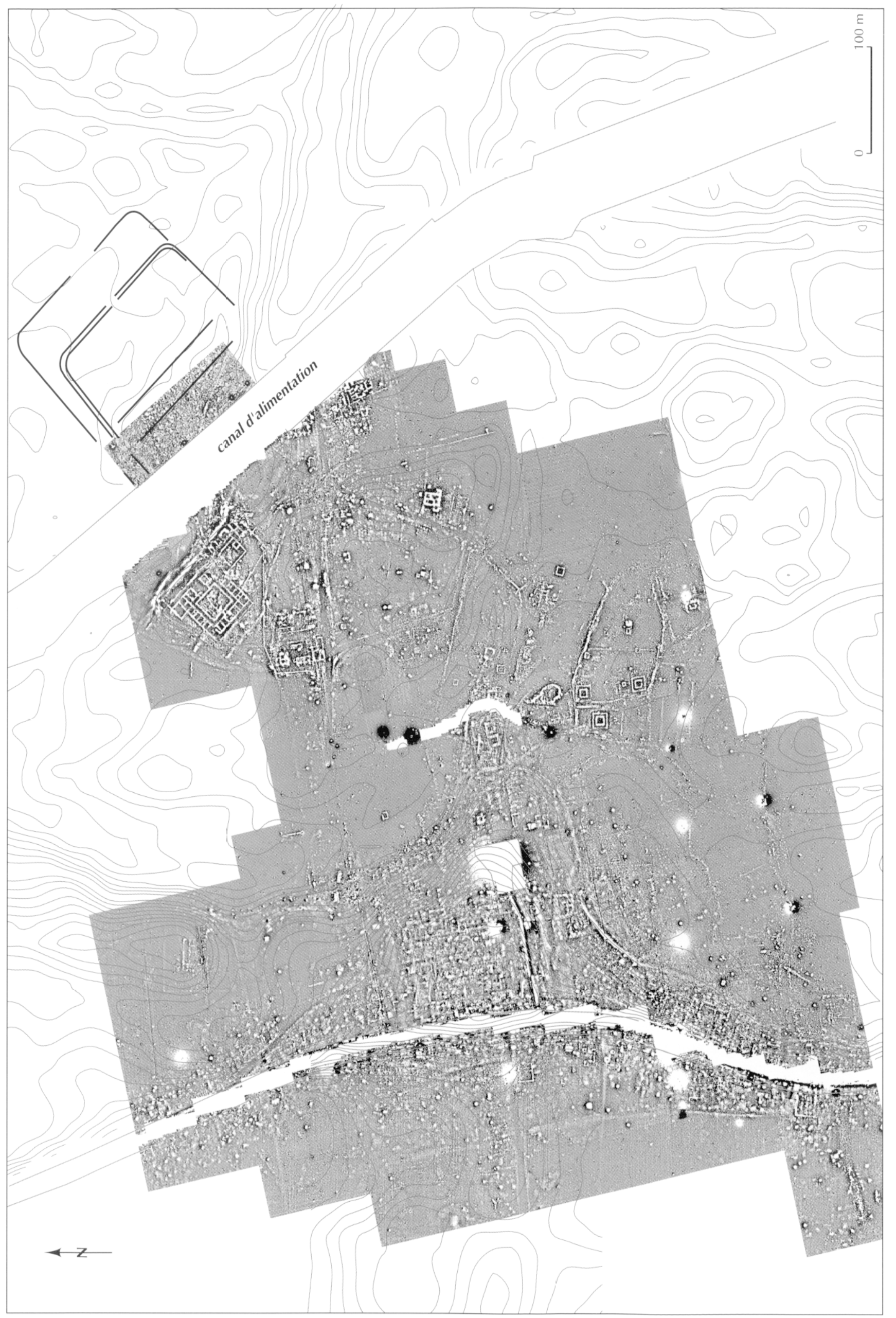




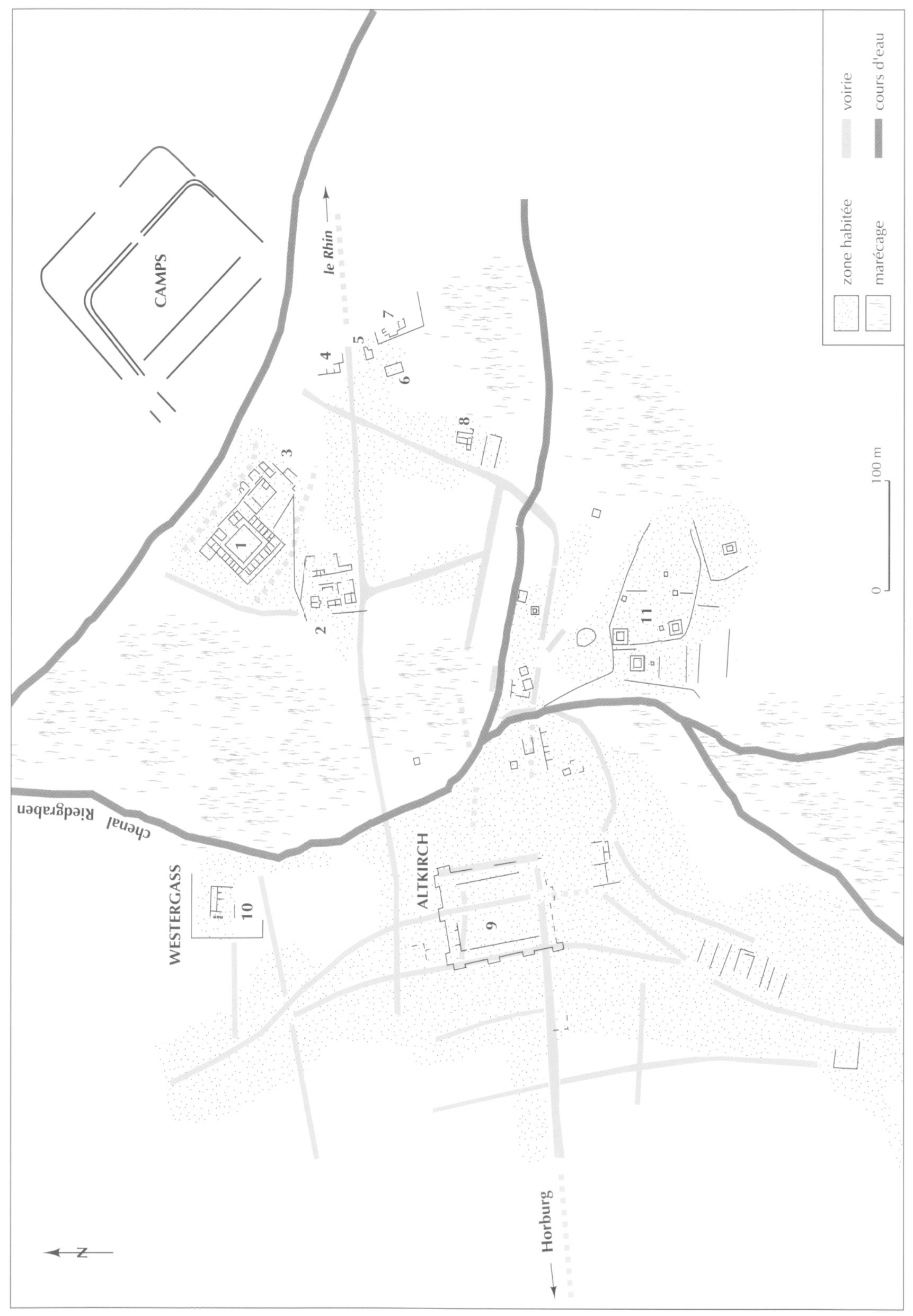

Gallia, 62, 2005, p. 215-277

(1) CNRS F́DITIONS, Paris, 2003 


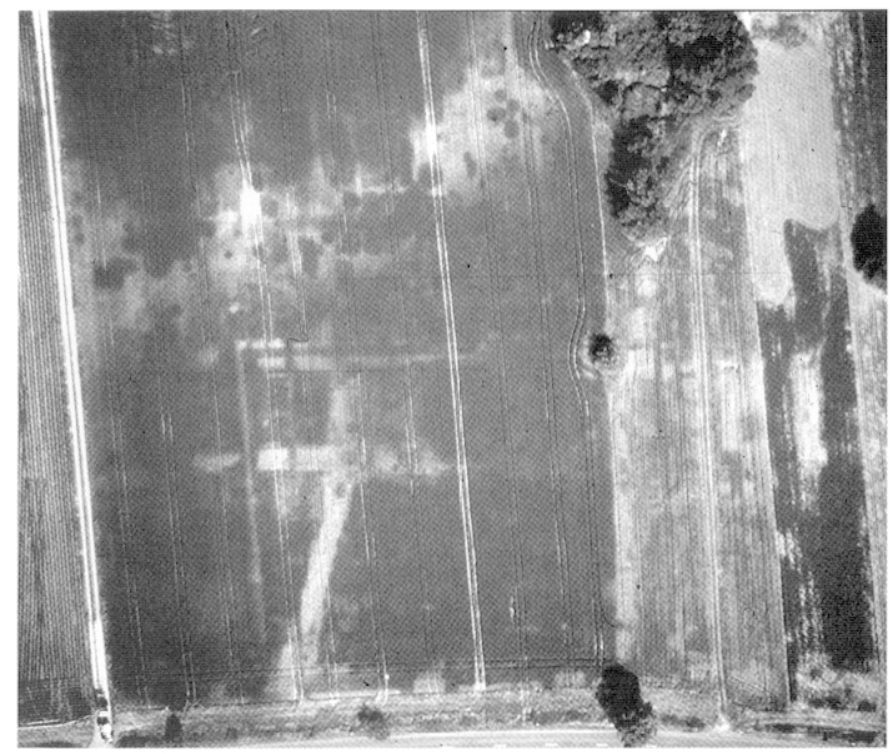

Fig. 7 - Le réseau de voirie sur la butte d'Altkirch (photo aérienne O. Braasch, Landesdenkmalamt Baden-Württemberg).

visible sur les photographies aériennes, est bordée par une série de fosses, alignées perpendiculairement à l'axe du chemin, ce qui témoigne sans doute de l'existence d'un habitat assez précoce. Plus à l'ouest a été reconnue une nécropole du BasEmpire (Biellmann, 2001). Lne grande voie (?) nord-sud semble marquer une des limites de l'agglomération, à une centaine de mètres à l'ouest de la route moderne.

Au sommet de la butte d'Altkirch, l'image géophysique révèle unc occupation très dense. Les fouilles de l'université de Freiburg ont montré que s'y était installé un palais forteresse de l'époque de Valentinien (9), auquel ont succédé plusieurs églises, dans l'angle sud-ouest de l'édifice (voir infra, p. 241-247). Ces structures tardo-antiques oblitèrent la voiric plus ancienne, que révèlent en revanche fort bien les photographies aériennes (fig. 7). On connaît ainsi plusieurs chaussées parallèles, recoupées par une voie oblique nord-ouest - sud-est qui forme avec les premières une figure en forme de " 7 ". Les sondages profonds ont montré une occupation qui remonte au tout début de l'époque julio-claudienne.

\section{ENSEMBLE 4}

Au nord de la butte d'Altkirch, une autre éminence (Westergass) est occupéc par un bâtiment 10 , où les fouilles de l'université de Fribourg-en-Brisgau ont mis au jour un praetorium routier, datable de l'époque constantinienne tardive (voir infra, p. 241).

\section{ENSEMBLE 5}

À l'est des buttes d'Altkirch et de W'estergass, l'étude des courbes de niveau révèle l'existence d'un chenal, encore actif avant le remembrement ("Riedgraben "). L.es fouilles de 2003 ont prouvé que ce dernier arait été au moins partiellement

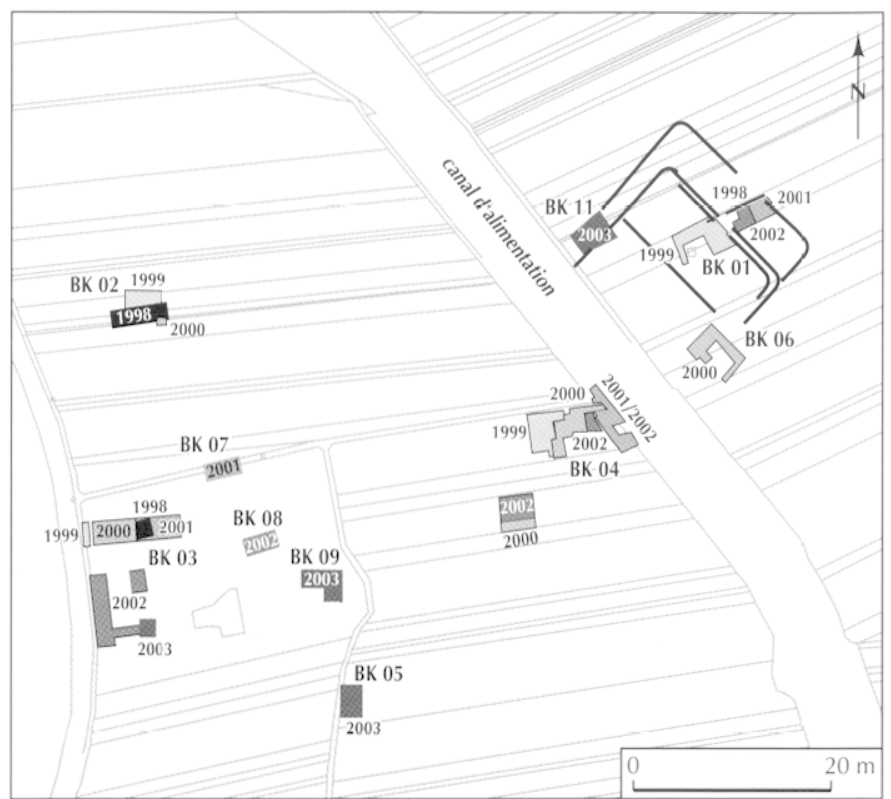

Fig. 8 - Plan général des fouilles effectuées depuis 1998 (dessin M. Reddé, LPHE).

canalisé, dès le I ${ }^{\text {er }}$ s. apr. J.-C. (voir infra, p. 235-237). À l'est du Riedgraben, l'image géophysique montre une zone de teinte grise quasi uniforme, dans laquelle n'apparait aucune structure anthropique. Les recherches actuelles y ont reconnu un vaste marécage.

\section{ENSEMBLE 6}

Au sud-est de la butte d'Altkirch, sur la rive orientale du Riedgraben, la prospection magnétique révèle la présence d'au moins quatre temples à cella centrale et déambulatoire (11). Ce " quartier religicux », à l'écart du reste de l'agglomération, est en cours de fouille par les soins de l'université de Bâle (voir infra, p. 238-240).

Le détail de l'organisation spatiale ne peut évidemment être commenté plus précisément dans le cadre limité de cet article. Il reste de toute façon hypothétique et supposerait de nombreuses recherches au sol pour être validé de manière définitive. Les grandes lignes apparaissent toutefois avec assez de clarté. Il est évident que le site antique était parcouru par d'assez nombreux chenaux et comprenait des zones basses marécageuses, ce que confirment l'étude géoarchéologique, les recherches environnementales et la fouille des structures. Cette topographic explique l'existence de quartiers séparés les uns des autres, et une organisation de la voirie qui n'est pas orthonormée. les premières fouilles montrent au demeurant que le système viaire n'est pas chronologiquement homogène et qu'il s'est constitué de manière progressive. Enfin, on perçoit asse\% bien, désormais, les principales limites spatiales de cette agglomération, même si quelques recherches géophysiques sont encore nécessaires, notamment au sud, en direction d'un diverticule routier où la photographie aérienne montre que se détache vers le sud-ouest une voie romaine mal connue, pour l'instant non précisément datée. 
Les recherches au sol, depuis 1998, ont porté sur des secteurs divers, de manière à évaluer les grands ensembles morphologiques et archéologiques du site (fig. 8). Nous en donnons ci-dessous un aperçu synthétique.

M. R.

\section{LES CAMPS JULIO-CLAUDIENS}

Le complexe militaire julio-claudien a été identifié grâce aux différentes couvertures aériennes, qui révèlent l'existence de deux enceintes rectangulaires concentriques (fig. 9). Les fouilles menées depuis 1998 ont montré que le "grand camp " précédait le plus petit ${ }^{4}$ (fig. 10). De ce fait, l'intérieur de la fortification primitive est largement oblitéré par les reconstructions ultérieures. En outre, le canal moderne semble avoir partiellement détruit les structures des deux édifices, ce qui ne permet pas de connaitre leur longueur. Les sondages, au sud du canal, ont toutefois prouvé que le complexe militaire ne s'étendait pas dans cette zone (voir infra, p. 231-232). En l'état actucl de nos connaissances, le "grand camp " mesure environ $175 \mathrm{~m} \mathrm{x}$ $200 \mathrm{~m}$ (minimum), avec une superficie d'au moins 3,5 ha, le "petit camp " occupe un espace de $130 \mathrm{~m}$ x $155 \mathrm{~m}$ (minimum), soit une surface d'au moins 2 ha. La forme générale des deux enceintes semble celle d'un rectangle régulier aux angles arrondis. Seule la disposition interne du "petit camp " commence à être connuc. Les photographies aériennes y révèlent en effet un réseau de voies internes parallèles (fig. 9) ; ces clichés sont toutefois trop peu explicites pour que l'on puisse identifier les différents bâtiments. Les fouilles ont mis au jour les trois portes conservées: au nord-est une ouverture à double vantail, au sud-est et au nord-ouest un passage unique. La position des deux portes simples permet de localiser l'axe de la via principalis. Il n'est en revanche pas aisé de déterminer avec certitude la position de la porte prétorienne. La présence d'un passage à double vantail, au nord-est, face au Barbaricum, ne suffit pas, notamment pour l'époque julioclaudienne, à identifier la porta praetoria à cet endroit ${ }^{5}$. On peut, en effet, envisager la possibilité d'une seconde porte double au sud-ouest, mais l'hypothèse est malheureusement invérifiable, puisque le canal d'alimentation a détruit cette partie du camp ${ }^{6}$. Seule l'identification des principia permettra de trancher cette question, et, à cet égard, deux solutions sont possibles (fig. 10). Une fouille prochaine derrait clarifier la situation.

L'articulation générale du "grand camp " reste très mal connue. Seule la position de la porte nord-est peut être approximativement localisée, grâce à l'axe de la voie intérieure, bordée de casernements, et réutilisée à l'époque du "petit camp ". Cette orientation du camp primitif diverge quelque peu, dans cette zone, d'avec celle des structures du camp postérieur. En revanche, dans les parages de la porte nord-ouest, cette différence d'axe semble nettement moins sensible.

4. Ce chantier est dirigé par J.J. Wolf.

5. Sur l'articulation interne de ces ourrages militaires et le vocabulaire afférent, voir désormais Reddé et al., à paraitre, notamment fig. 18. 6. À titre de comparaison, rappelons que la porte décumane du camp de Krefeld-Gellep est double (Fahr, Reichmann, 2002).

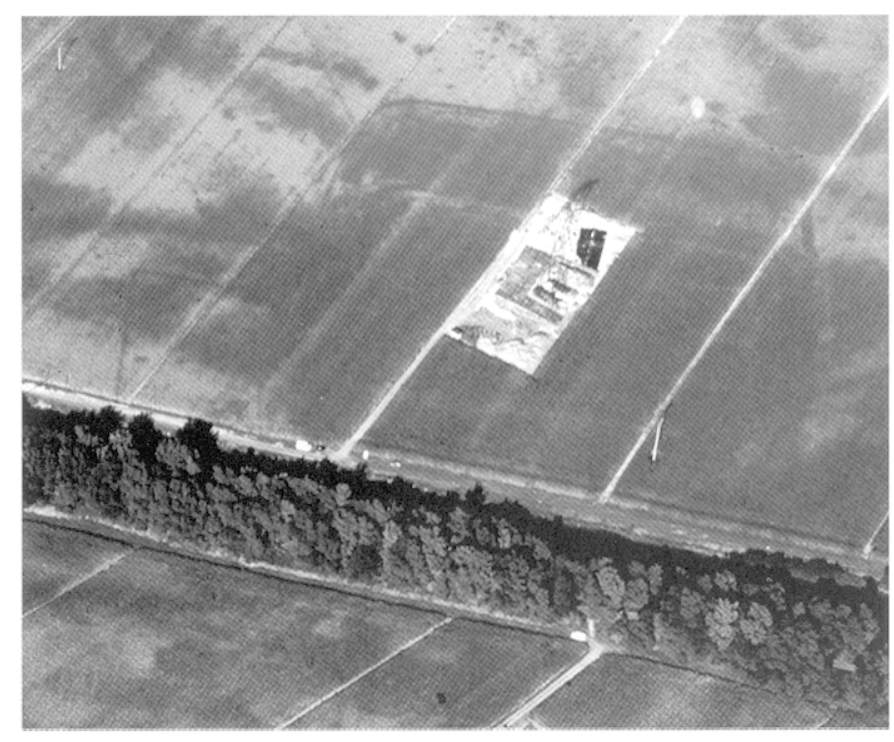

Fig. 9 - Les camps julio-claudiens pendant les fouilles de 1999 (jhoto aérienne R. (ioguey).

Les photographies aériennes ne révèlent clairement qu'un seul fossé autour du "grand camp " (fig. 9). L'unique fouille menée, au nord-est, sur ce système défensif n'a pas permis de déterminer la nature du rempart, dont les boisements n'ont pu être reconnus, à l'exception possible d'une tour (fig. 11). À sa place a été observé un deuxième fossé intérieur, dont on ne saurait affirmer qu'il a fonctionné en même temps que l'autre. La seule structure bien identifiée, pour l'instant, est un groupe de deux casernements séparés par une ruelle. Il s'agit très clairement de constructions sur sablières basses de bois, dans lesquelles on distingue un double système d'antichambres (environ $3 \mathrm{~m} \mathrm{x} 2 \mathrm{~m}$ ) ouvrant sur la rue, suivies, à l'intérieur, d'une série de chambrées (environ $4 \mathrm{~m} \times 3 \mathrm{~m}$ ). Lne telle disposition, caractéristique des baraquements militaires (Reddé el al., à paraître), révèle en outre, à l'extrémité de chaque bloc, vers le rempart, une structure plus grande, avec des subdivisions internes, où l'on reconnaît aisément le logement du centurion. La longueur des baraques, coupées au sud par le fossé du "petit camp ", n'est pas connue. Une série de dépotoirs, de latrines, de silos et de puits est venue parasiter l'ordonnancement initial de cet ensemble. D'autres casernements ont été observés près de la porte nord-onest, mais leur fouille est restée incomplète.

Le "petit camp " a révélé la présence de deux fossés contemporains. Au-delà de la porte nord-ouest, toutefois, le fossé externe fait défaut (fig. 12). Le rempart, large d'un peu plus de $4 \mathrm{~m}$ sur le front nord-est, ne dépasse guère $2,75 \mathrm{~m}$ près de la porte nord-ouest. Dans les deux cas, il s'agit d'un rempart en terre sous coffrage de bois (fig. 13). Ce demier repose sur des poutres longitudinales basses, apparemment sans tirants transversaux au niveau du sol, mais l'architecture peut avoir été différente en élévation. La porte nord-ouest, large de $3 \mathrm{~m}$ environ, est flanquée de deux tours carrées d'environ $3 \mathrm{~m}$ x $3 \mathrm{~m}$, à quatre poteaux (Manning, Scott, 1979, type IIa). La porte nord-est, double, présentait, dans un premier temps, une largeur d'au moins $6,50 \mathrm{~m}$, et était cncadréc par deux tours 


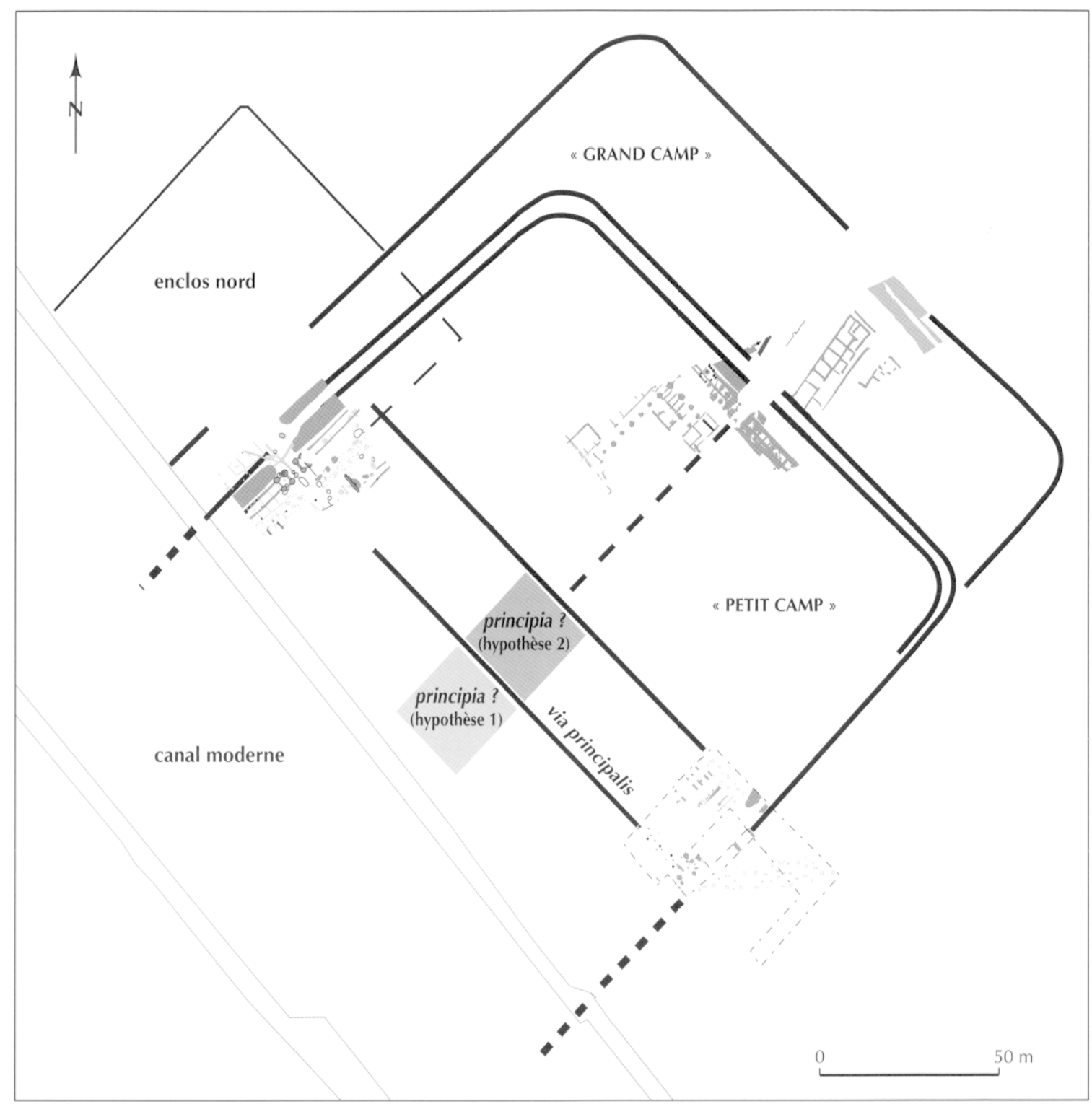

Fig. 10 - Plan d'ensemble des camps julio-claudiens (dessin M. Reddé, EPHE).

carrées de 2,50 m-3 m (Manning, Scott, 1979, type IIb) ${ }^{7}$. Dans un second temps, ce passage a été rétréci pour ne plus laisser place qu'à une entrée simple, large d'environ $4 \mathrm{~m}-4,50 \mathrm{~m}$. On ne connaît pas les tours intermédiaires.

À l'intérieur; deux bâtiments ont été fouillés dans les parages de la porte nord-est, immédiatement derrière la via sagularis: un petit horreum de $12 \mathrm{~m}$ x $4 \mathrm{~m}$ environ, ancré sur quatre paires de puissants poteaux, avec des tirants transversaux dans lesquels s'implantent des boisements verticaux de section circulaire, très serrés (Reddé et al., à paraître, fig. 101) ; un magasin à cour centrale, d'environ $25 \mathrm{~m} \mathrm{x} 20 \mathrm{~m}$, dont seule la partic septentrionale a pu être fouillée (fig. 13). Il s'agit d'unc construction sur sablières basses en bois, avec un portique

7. Il est toutefois possible que la tour nord ait compris une plate-forme à six poteaux. sur les grands côtés de la cour. L’identification repose, pour l'essentiel, sur la similitude du plan avec des bâtiments de même type connus en Rhénanie (Petrikovits, 1975, fig. 19; Reddé et al., à paraître, fig. 102).

La chronologie de cet ensemble militaire n'est pas très aisée à déterminer dans le détail. Le matériel céramique augustéen et augusto-tibérien précoce fait totalement défaut ; le matériel flavien est rare. Dans la mesure où l'on peut mener l'étude sur une base statistique asse\% réduite, secteur par secteur, et en tenant compte des diverses réfections locales, on proposcra deux tableaux montrant la chronologic du mobilier céramique dans les casernements du " grand camp " (fig. 14) et près de la porte nord-ouest du "petit camp " (fig. 15).

Étudiant les monnaies des camps, en 2001, L. Popovitch avait présenté un histogramme qui doit aujourd'hui être quelque peu modifić au vu de la datation des contremarques 


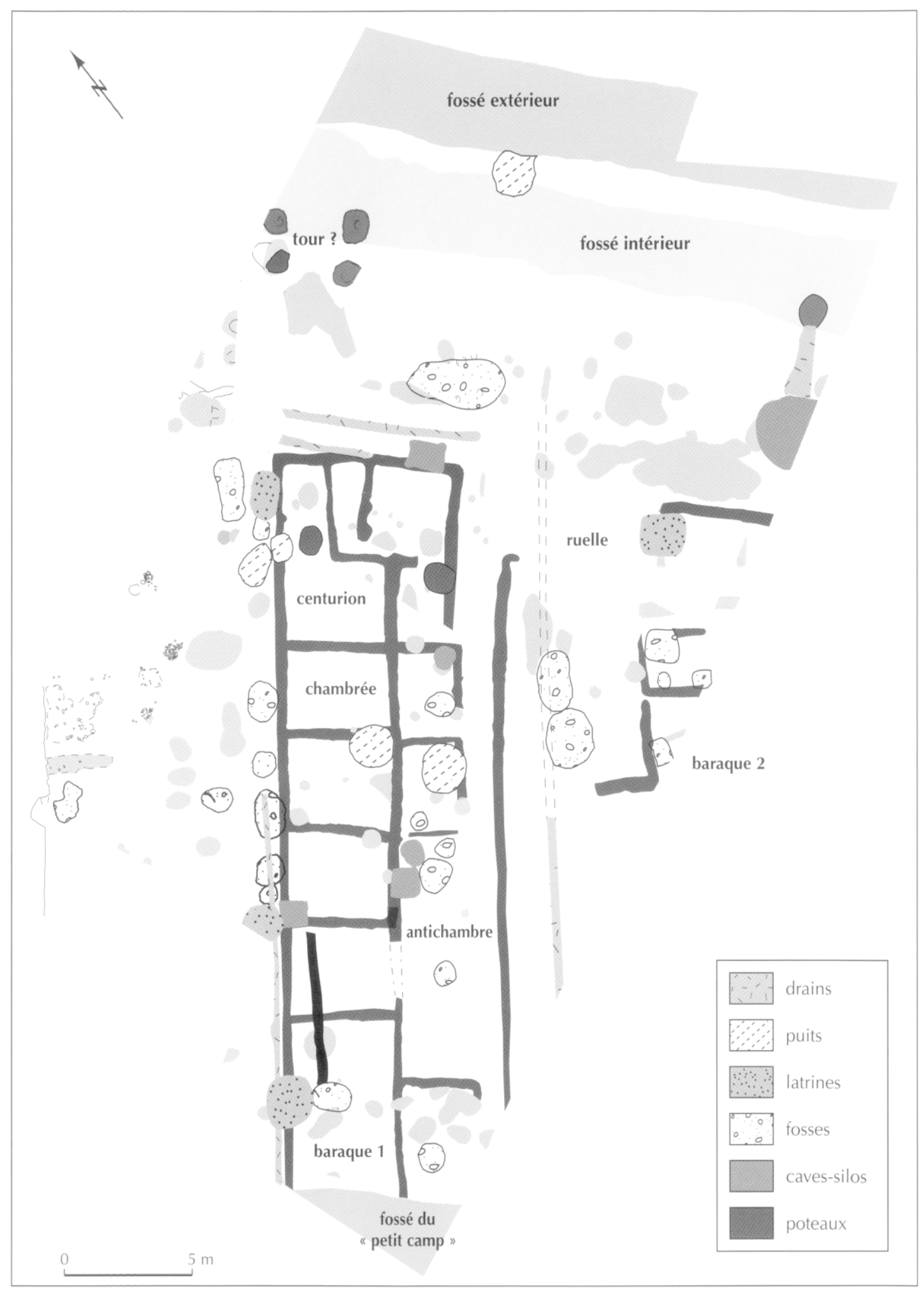

Fig. 11 - Plan des structures du "grand camp" près de la porte nord-est (dessin M. Reddé, EPHE et J.J. Wolf, Service départemental archéologique du Haut-Rhin). 


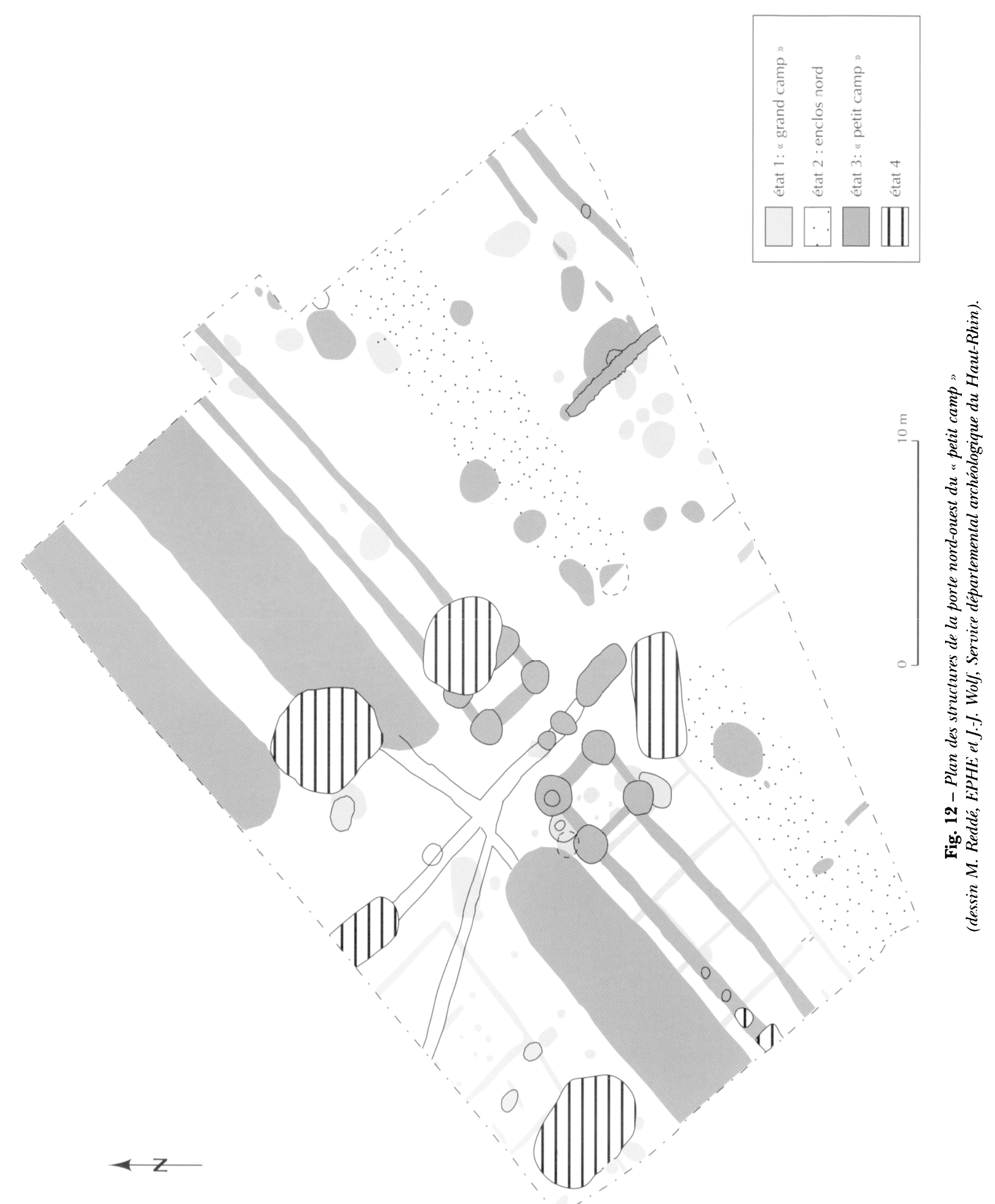





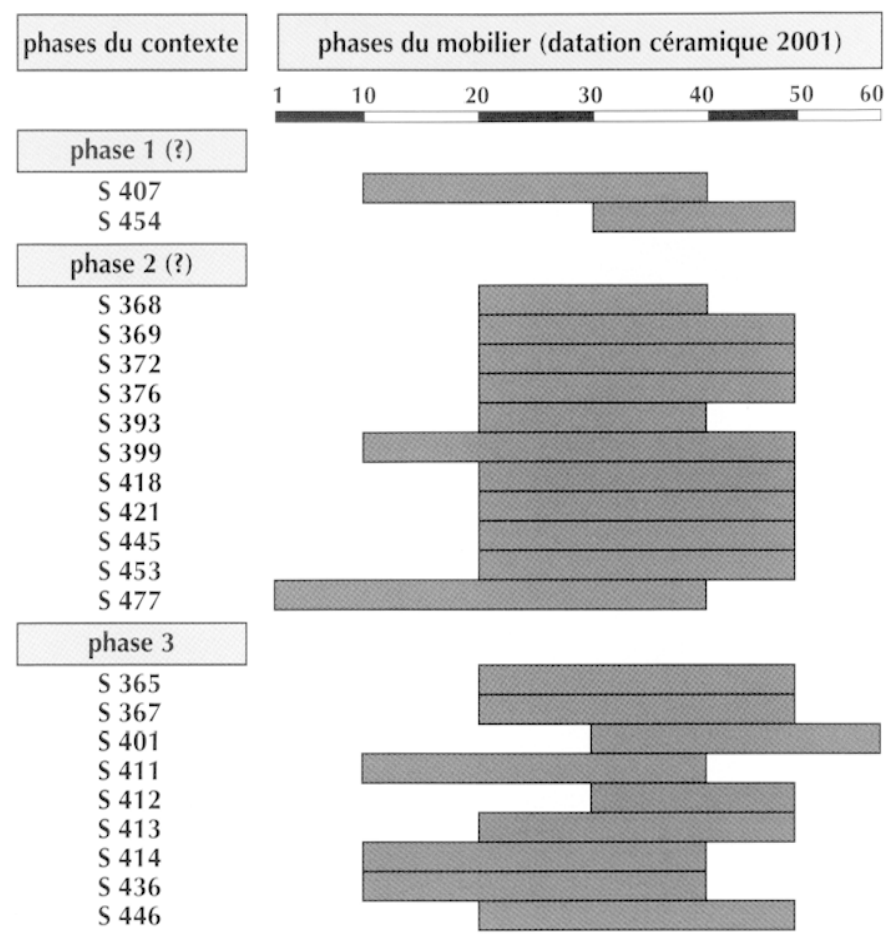

Fig. 14 - Diagramme chronologique de la céramique fouillée dans les baraques du "grand camp ", près de la porte nord-est (DAO B. Viroulet, Service départemental archéologique du Haut-Rhin).

récemment proposée par $\mathrm{R}$. Martini (Popovitch in Nuber, Reddé et al., 2002, fig. 22 ; Martini, 2003). La statistique présentée ici porte sur 596 monnaies datées avec précision entre 49 av. J.-C. et 199 apr. J.-C. On observera, au sein du stock augusto-tibérien, nettement prépondérant, l'imporance de la décennie 20-29. Remarquable aussi est la proportion des émissions datées entre 30 et 49 , ainsi que le petit stock des pièces néroniennes et flaviennes précoces (fig. 16).

On ne peut manquer de rapprocher ces données de la présence des très nombreuses tuiles estampillées au timbre de la $\mathrm{XXI}^{\mathrm{e}}$ légion, présentes dans l'agglomération civile. Ces tegulae appartiennent toutes aux types julio-claudiens d'une troupe qui arrive à Vindonissa sous Claude (Wiegels, 1983). Il est toutefois difficile de déterminer si les camps d'Oedenburg ont été réellement construits par cette légion, ou si une autre unité a pu être présente antérieurement sur le site, dès l'époque tibérienne : le matériel ne donne pas, pour l'instant, une réponse parfaitement claire, mais chaque campagne livre davantage de céramique du deuxième quart du I ${ }^{\mathrm{er}} \mathrm{s}$. apr. J.C. Il convient de rappeler que l'habitude d'estampiller les tuiles n'apparaît que sous Claude, de sorte qu'une occupation militaire avant cet empereur peut fort bien n'avoir laissé aucune trace épigraphique. À titre d'hypothèse de travail, nous suggérerons la présence d'une unité à Oedenburg du règne de Tibère jusqu'à la conquête des Champs Décumates, au tout début de l'époque flavienne. Cette proposition remplace celle que nous avions initialement émise d'un camp construit sous

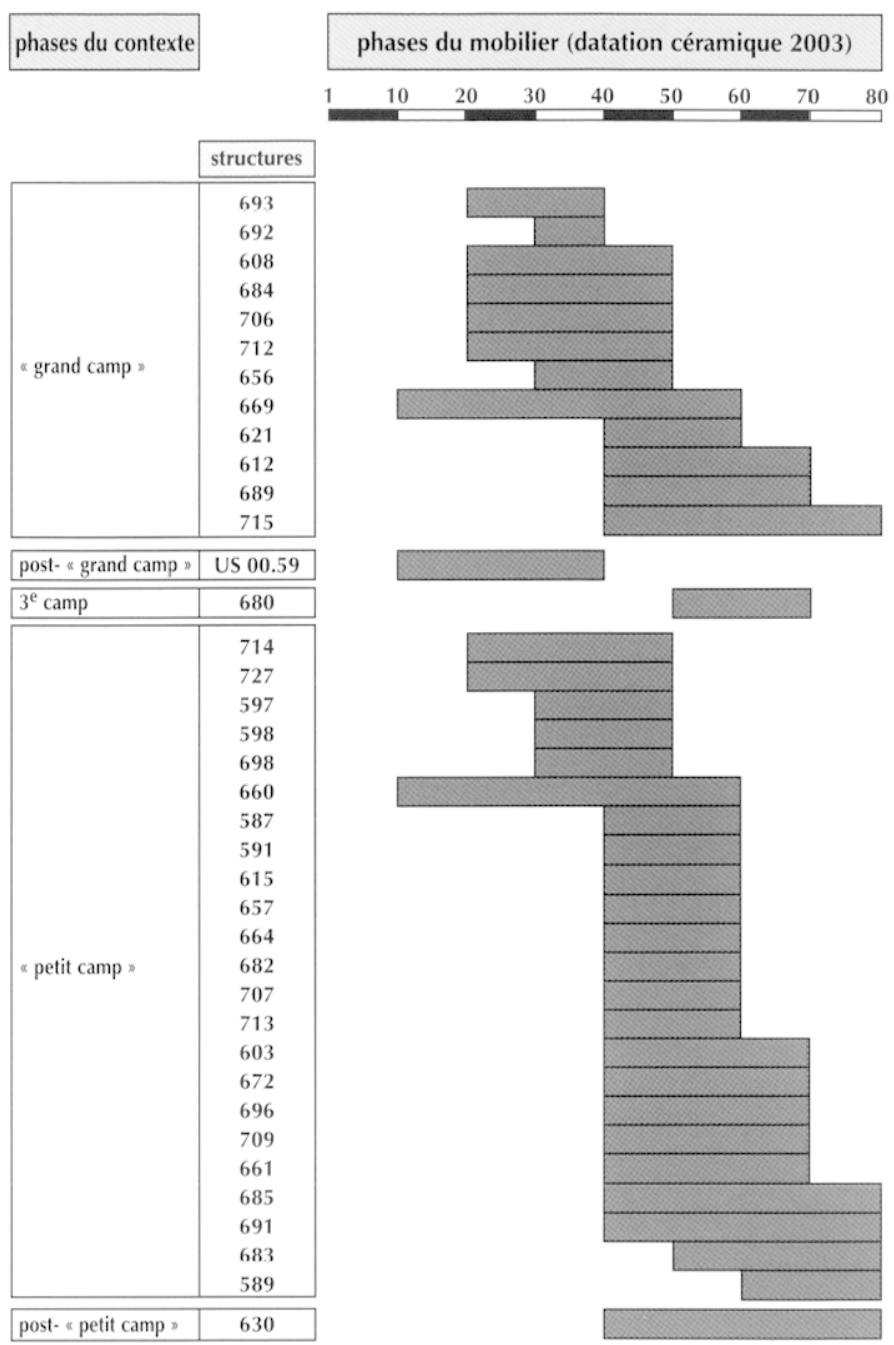

Fig. 15 - Diagramme chronologique de la céramique fouillée près de la porte nord-ouest du "petit camp " (DAO B. Viroulet, Service départemenlal archéologique du Haut-Rhin).

Claude. De son côté, l'étude des militaria métalliques laisse supposer une garnison de légionnaires, en raison de la découverte d'une vingtaine de garnitures de lorica segmentata, mais le cantonnement concomitant d'auxiliaires ne saurait être exclu (Fort, 2000).

M. R., B. V., J.J. W. et L. P.

\section{L'AGGLOMÉRATION GIVILE}

Nous avons tracé ci-dessus les grands traits topographiques de l'agglomération civile, où plusicurs fouilles ont été menées depuis 1999. Ne pouvant entrer ici dans le détail de ces recherches, nous évoquerons successivement trois exemples d'occupation (fig. 8) : les installations à la sortie occidentale dı "petit camp" (chanticr BK 04), une zone d'habitat installée en bordure des cours d'eau qui traversent le site (chantier BK 09) et un temple de tradition indigène (chantier BK 05). 


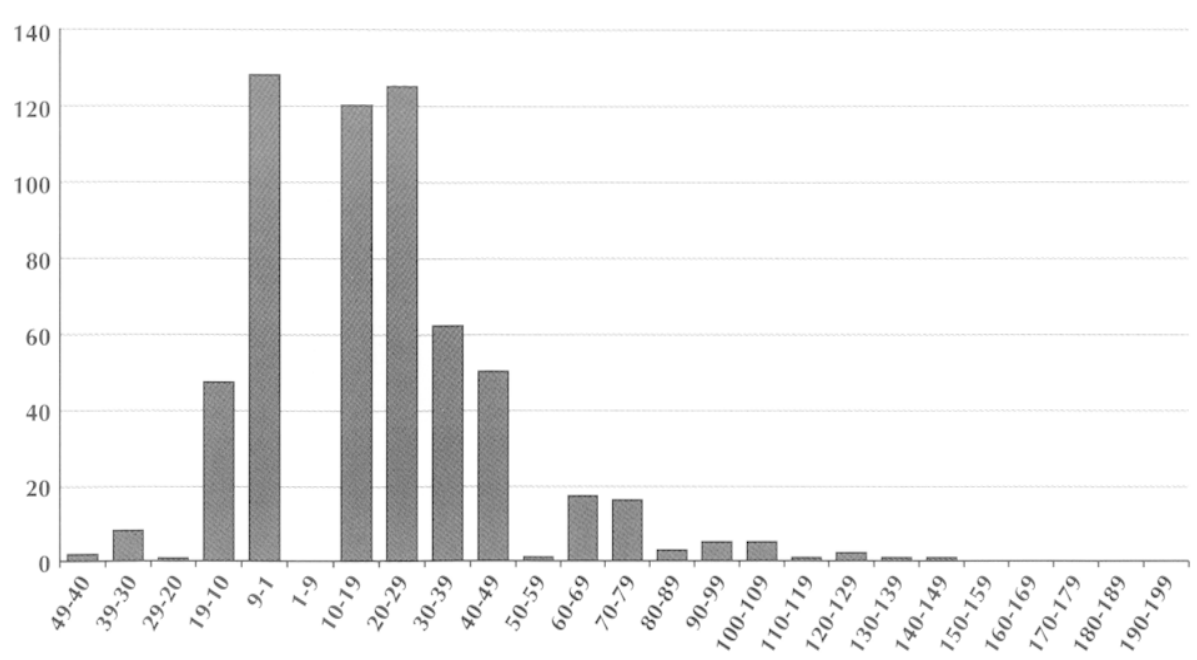

Fig. 16 - Histogramme des monnaies des camps (d'après Popovitch, in Nuber, Reddé et al., 2002, fig. 22).

\section{LES ABORDS DU CAMP JULIO-CLAUDIEN}

L'espace naturel, à la sortie de la porte sud-ouest du « petit camp ", est caractérisé par la présence d'un ancien chenal, aujourd'hui asséché ${ }^{8}$. Le canal moderne en a réutilisé partiellement le cours et on peut douter, dans ces conditions, que le camp ait été protégé par un fossé dans ce secteur. Un peu audelà, vers le sud-ouest, la fouille a mis au jour l'existence d'un autre paléochenal, approximativement parallèle au rempart du camp. Ce bras n'était probablement pas en eau de manière permanente. Son tracé a, en effet, été largement réoccupé par des séries de constructions ultérieures (fig. 17). Il constituait toutefois une zone basse, marécageuse, sans doute fréquemment inondée, où nous avons pu observer divers niveaux de crues (voir infra, p. 249-252).

\section{Le I ${ }^{\text {er }}$ s. apr. J.-C.}

Dans une première phase de l'occupation, l'homme a tenté d'aménager cet espace humide en installant un peu partout des claies, des fascines de brindilles, des planchers, qui permettaient de traverser à peu près à pied sec (fig. 18a et b). Un peu plus à l'ouest, une barre de graves, orientée nord-est - sudouest a favorisé l'implantation d'une voie qui conduisait très vraisemblablement à la porte du camp. Cette chaussée, large d'environ $4 \mathrm{~m}$, est simplement constituée d'un lit de graviers à la sortie de la forteresse. Plus au sud, en revanche, les observations effectuées lors des fouilles de l'université de Bâle ont montré une séquence plus complexe qui débute vers 50 apr. J.-C. ${ }^{9}$. On observe d'abord un épisode de crue, avant une série de recharges qui se poursuivent jusqu'au début du ${ }^{\mathrm{I}} \mathrm{s}$. Les graviers ont été extraits du sous-sol voisin, laissant place à de grosses fosses, devenues à leur tour des dépotoirs.

Peu de bâtiments construits ont été observés dans cette première phase de l'occupation, à l'exception d'une série de sablières basses qui forment, le long de la voie, un petit

8. La fouille a été dirigée en 1999 par. M. Reddé assisté de A. Gelot, et en 2000-2002 par M. Reddé et J. Pellissier.

9. Ia fouille de ce secteur a été dirigée par F. Siegmund, assisté de J. Wininger (2000), puis par C. Schucany et P.-A. Schwarz (2002). bâtiment allongé sans plan bien identifiable (bâtiment 2). On observe, en revanche, la présence d'assez nombreuses fosses qui témoignent d'activités diverses. Parmi celles-ci, on doit noter quelques structures particulières (fig. 17).

La fosse a (01-04-75) contenait essentiellement des os longs de bœuf, brisés de manière à récupérer la moelle, soit pour des raisons culinaires, soit plutôt pour l'assouplissement des cuirs.

La structure b (01-04-50), formée de branchages et de planches de pin, présente une forme de navicelle $(4,30 \mathrm{~m}$ x 1,25 m) (fig. 18a). Installée en bordure du paléochenal, sans doute dans une zone perpétuellement humide, au milieu de copeaux de bois, elle contenait notamment, au milieu d'un riche matériel paléobotanique, quelques fragments d'écorce de bouleau. Sa forme ouverte n'en fait certainement pas une nasse, et sa structure interne, sans charpente, exclut une barque. On pense plutôt à une cuve destinée à une activité artisanale qui reste à déterminer (rouissage ?).

Les autres fosses ont servi de dépotoirs. C'est le cas, en particulier, d'une vaste couche $\mathbf{d}$ organique, noire, lenticulaire, qui s'étend à la sortie du camp, le long de la voie, ou de la fosse e, attribuable à la seconde moitié du $\mathrm{I}^{\mathrm{er}} \mathrm{s}$. Il en va de même d'une très grosse fosse $\mathbf{f}$, installée le long de la voie. Le matériel, très abondant, montre un remplissage vers la fin du règne de Néron (sondage de G. Matter, université de Bâle, en 1999). Les fosses g et $\mathbf{h}$, elles aussi le long de la voie, témoignent d'une chronologie identique.

À environ $100 \mathrm{~m}$ vers le sud, ont été mis au jour, des deux côtés de la voie, des vestiges de maisons à sablières basses et parois en torchis qui semblent avoir brûlé après le milieu du $\mathrm{I}^{\mathrm{er}} \mathrm{s}$. (fig. 19). Leurs restes ont été découverts dans le comblement de plusieurs fosses. Un puits était associé à ces structures. Ensuite a été creusé un fossé sur le flanc occidental de la voie, et un fossé sur son flanc oriental. Plusieurs trous de poteaux attestent l'existence de bâtiments de part et d'autre de la chaussée. Ces structures une fois comblées, le secteur a vu l'installation de petits ateliers pour la métallurgie du fer. On a pu observer deux enclumes au nord de la voie, et quelques dépotoirs de forge au sud, ainsi qu'une couche noire, riche en scories de fer et en battitures. D'autres fosses ont livré le gravier nécessaire à une seconde recharge de la voie. Elles sont datées 


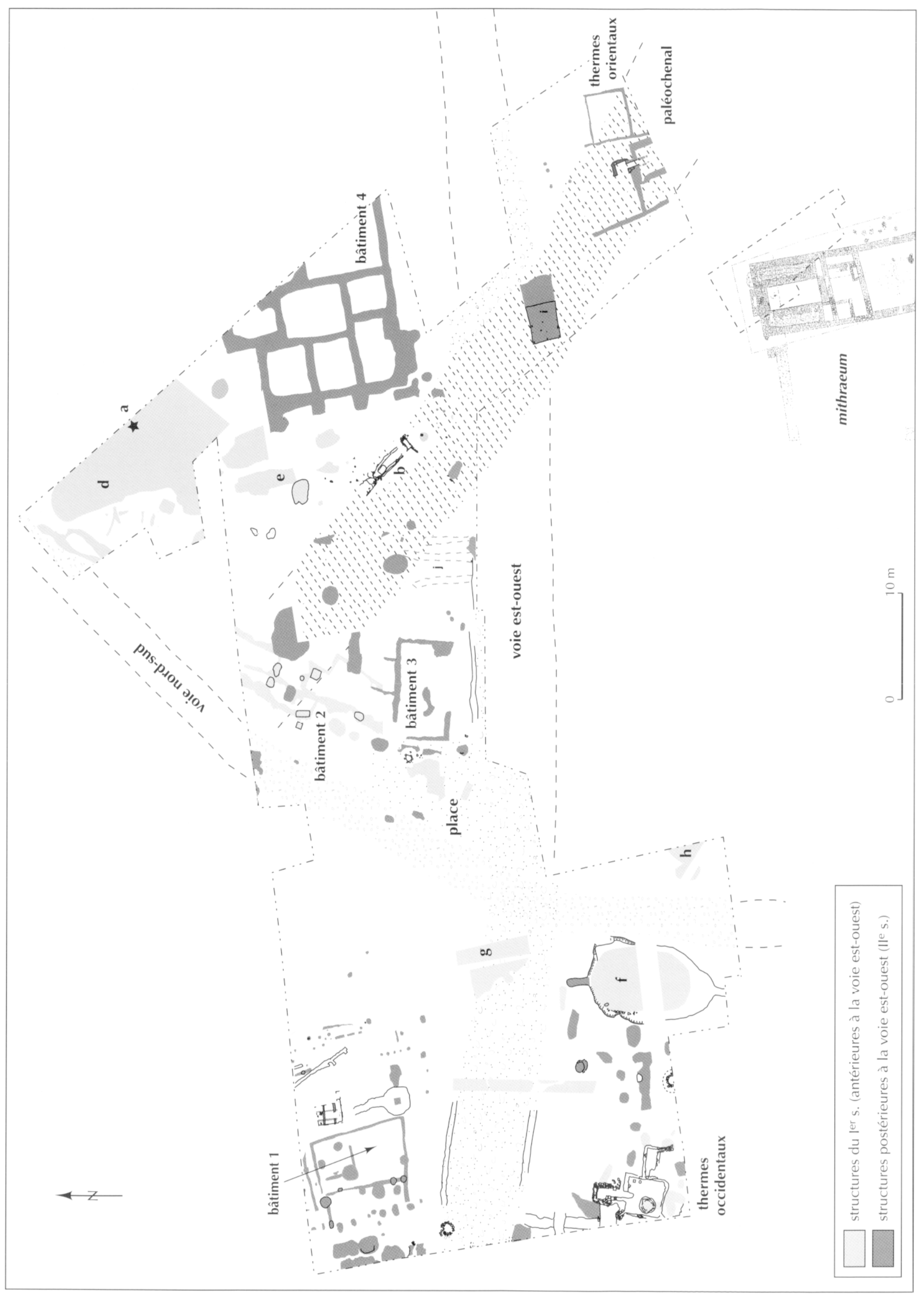

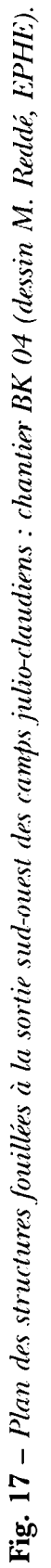


de la fin du ${ }^{\text {er }}$ s., d'après le mobilier qui les comble. Tout ce quartier semble avoir été abandonné vers le début du $\mathrm{II}^{\mathrm{e}} \mathrm{s}$.

Au total, toutes ces installations pourraient avoir été liées aux activités industrielles du camp, mais on observera que l'étude fine du matériel n'est pas achevée et qu'il n'est pas aisé de distinguer ce qui est exactement contemporain de la présence militaire de ce qui la suit immédiatement. On ne

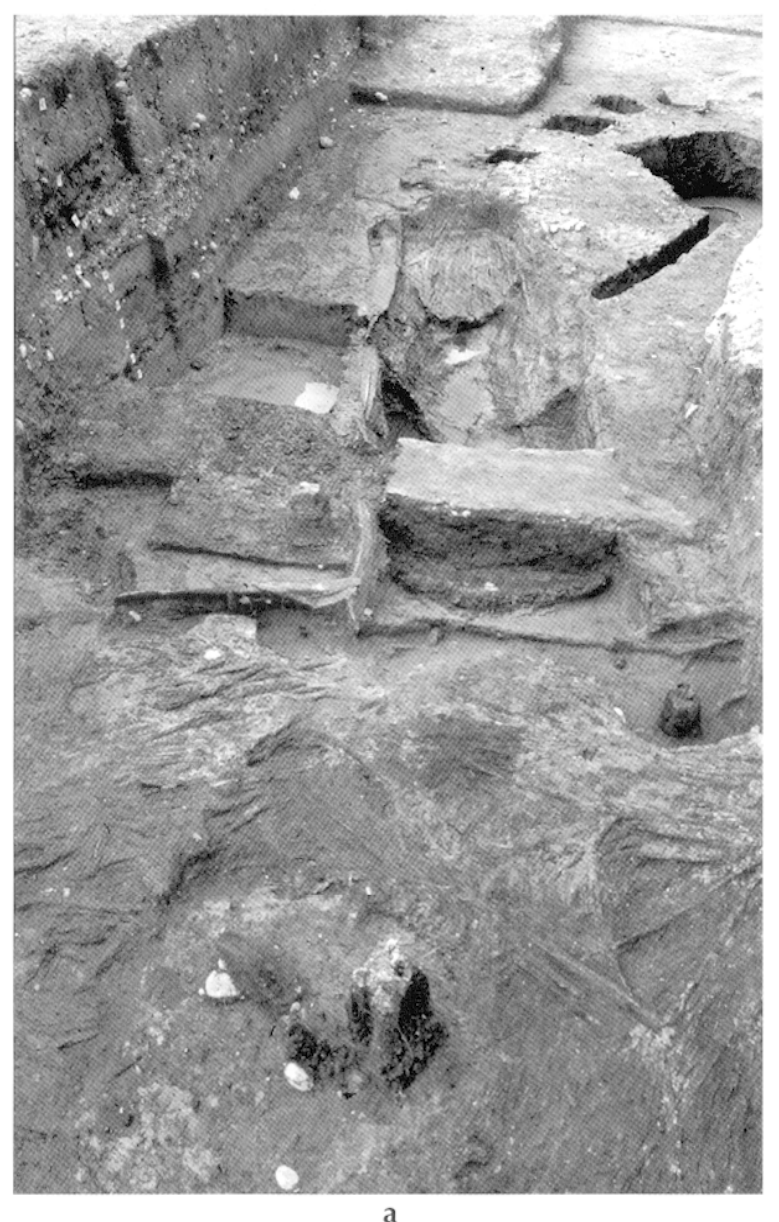

a

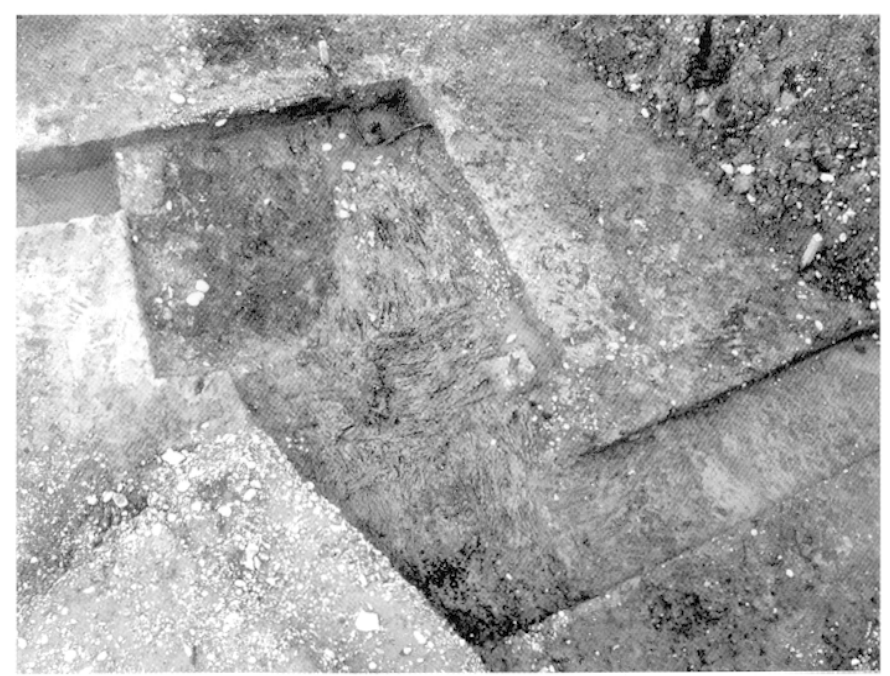

b constate pas, en tout état de cause, l'installation d'un véritable habitat groupé dans ce secteur. On note aussi que le premier ensemble thermal de cette zone, le long de la voic est-ouest de la fin du $\mathrm{I}^{\mathrm{cr}}$ s., est installé sur une fosse-dépotoir datable de l'époque flavienne, à l'ouest de la zone fouillée (" thermes occidentaux "). De taille très modeste, il paraît postérieur à l'occupation militaire.

\section{Le II ${ }^{\text {e }}$ s. apr. J.-C.}

La phase suivante voit une réorganisation de ce secteur autour d'une nouvelle voie est-ouest qui traverse tout le site depuis le piémont nord d'Altkirch. La suite de son tracé, vers le Rhin, n'est pas connue pour l'instant. Une fouille limitée, en contrebas de la butte d'Altkirch, a montré que la chaussée était associée à des pieux qui la stabilisent latéralement dans la traversée du Riedgraben. Ces bois ont été abattus dans l'hiver 97-98 (datation O. Girardclos, Cèdre). Vers la même époque, les abords du camp, désormais abandonné et en ruines, font l'objet d'un réaménagement par apport de graviers sur les zones basses, notamment dans la traversée du paléochenal par la nouvelle voie. Celle-ci sera l'objet, au cours du temps, d'une série de recharges locales qui l'élargissent sensiblement, notamment vers le nord. Plusieurs bâtiments de nature différente apparaissent alors.

\section{Les bâtiments sur sablières de bois et poteaux (fig. 17)}

$\mathrm{Au}$ nord-ouest de la zone fouillée apparaît un ensemble d'environ $9 \mathrm{~m}$ x $9 \mathrm{~m}$ (bâtiment 1) qui comprend, à l'ouest, une sorte d'auvent porté par une série de petits poteaux de bois. Cet auvent ouvre sur un espace où plusieurs fosses-dépotoirs ont été découvertes, sans doute à l'emplacement d'une ruelle. Fn arrière (vers l'est) apparaît un espace rectangulaire subdivisé en trois parties (une grande pièce de $5 \mathrm{~m} \times 5 \mathrm{~m}$ environ, un couloir, une petite pièce de $3,50 \mathrm{~m}$ x 3,50 m). Plus à l'est encore ont été découverts un puits carré à cuvelage de bois et une cave, dont on ne peut dire s'ils dépendent ou non du bâtiment 1. Les dépotoirs contenaient un matériel de la fin du $\mathrm{I}^{\mathrm{cr}} \mathrm{s}$. au milieu du II ${ }^{\mathrm{C}} \mathrm{s}$. ; la chronologie de la céramique contenue dans la cave descend en revanche jusqu'au début du III $^{\mathrm{e}} \mathrm{s}$.

Au sud de la voie a été mise au jour une structure rectangulaire $\mathbf{i}(3,50 \mathrm{~m} \mathrm{x} \mathrm{2,80} \mathrm{m)}$ installée sur des pilotis de chêne qui portaient un plancher de sapin, effondré (fig. 20). Il s'agit probablement des restes d'un édifice plus vaste qui bordait la voie, mais a été partiellement enterré, une fois effondré, par ses ultimes recharges. Les pilotis ont été abattus dans l'hiver 145-146. L'ensemble a servi secondairement de dépotoir et de latrine.

Un même type de constructions sur pilotis apparaît au nord de la voie, mais nettement moins bien conservé (j). Les bois ont été enfoncés à travers les couches inférieures, jusqu'au lit du paléochenal. L'un d'eux a pu être daté postérieurement à 168 .

Fig. 18 - Exemple d'aménagement de sol humide: a, aver des brindilles (on apersoit au fond une structure de bois en forme de naricelle, sans doute à vocation artisanale); $b$, avec des claies (voir b, fig. 17) (clichés M. Reddé, EPHE). 


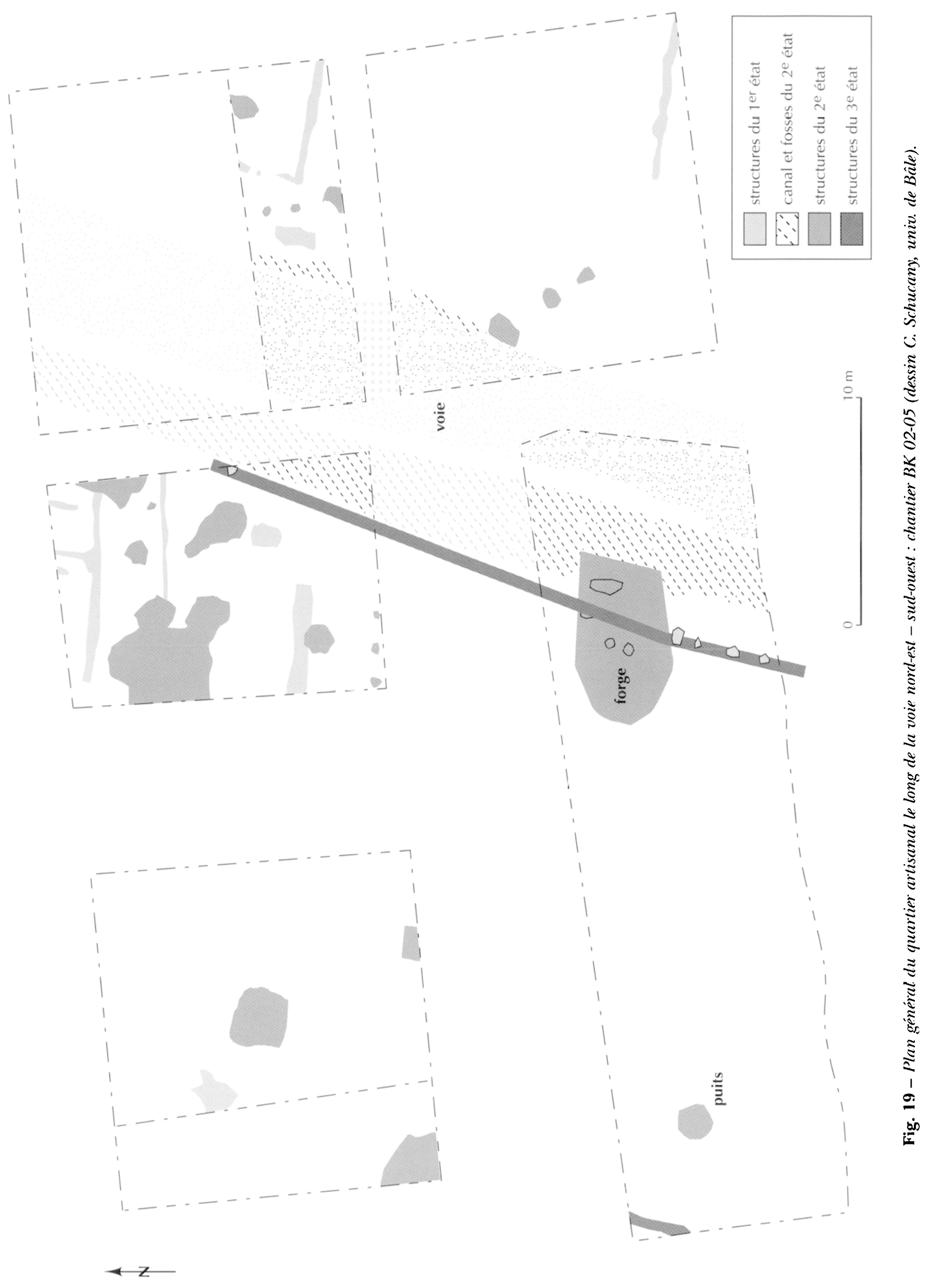




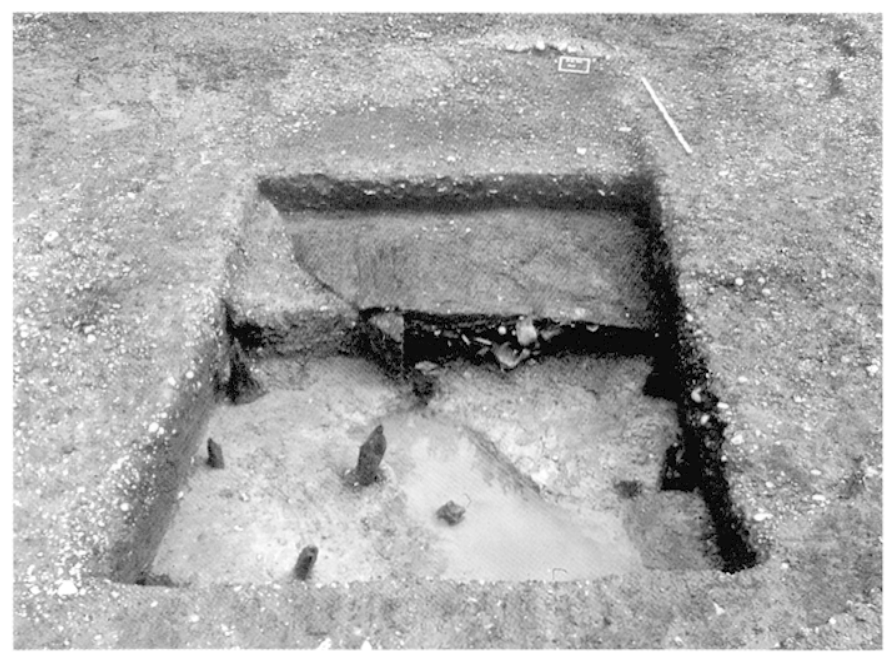

Fig. 20 - Structure sur pilotis : chantier BK 04 (voir i, fig. 17) (cliché M. Reddé, EPHE).

\section{Les bâtiments sur soubassement de pierre (fig. 17)}

Seul un bâtiment de ce type a été mis au jour (bâtiment 3 ). Il s'agit d'un rectangle de $9 \mathrm{~m} \times 6 \mathrm{~m}$, en blocs de basalte assemblés à sec. Compte tenu du type de construction, on doit sans doute envisager une élévation en pans de bois et torchis. L'espace est ouvert sur la voie est-ouest. Ni le plan du bâtiment ni le matériel recueilli, très peu abondant, ne permettent de déterminer avec précision la nature du bâtiment (échoppe ?). On notera que la voirie, immédiatement à l'ouest, forme désormais une espèce de place triangulaire, par simple élargissement du carrefour des deux rues.

\section{Les bâtiments en pierre (fig. 17)}

Plusieurs édifices apparaissent dans cette zone. Le bâtiment 4 est en partie coupé par l'emprise du canal. Dans son état actuel, il mesure environ $19 \mathrm{~m}$ (nord-sud) et sa dimension est-ouest dépassait les $20 \mathrm{~m}$ actuellement conservés. Il s'agit donc d'un édifice barlong, contreforté par une série de quatre pilastres espacés d'environ $4,50 \mathrm{~m}$ (soit une quinzaine de pieds, d'axe en axe) sur sa face ouest, ce qui laisse supposer une hauteur d'au moins deux étages, probablement avec des poussées latérales assez fortes. Au sud, en revanche, n'apparaissent, en dehors du pilastre d'angle, que deux contreforts.

Le bâtiment a été spolié. Le démontage des seules structures conservées, au sud, montre une fondation en tranchée assise directement sur le substrat, et constituée de pierres irrégulières, brutes de taille, extraites des carrières basaltiques du Kaiserstuhl, jetées à sec dans la tranchée. La couche d'épierrement, située au-dessus, révèle un mélange de gravats, tuileaux et mortier de chaux blanc pulvérisé, fragments de pierre, ce qui laisse envisager une élévation en blocage parementé, avec une couverture en tuiles, sans que des éléments significatifs d'architecture permettent d'en dire davantage. La largeur moyenne des tranchées d'épierrement (environ $0,80 \mathrm{~m}$ ) ne permet d'estimer celle des murs que de façon approximative. L'identification de ce grand bâtiment échappe à une analyse immédiate, compte tenu du fait que son plan est largement incomplet. Son architecture ne l'apparente pas à un édifice religieux, mais plutôt à un édifice de stockage, compte tenu de la présence de contreforts. Aucun matériel significatif n'a été récolté.

Plus au sud est connu un mithraeum, sondé dans les années 1970 , et au sujet duquel nous renvoyons à la bibliographie existante (Pétry, Kern, 1978). Le matériel de fouille n'ayant été récupéré que récemment, sa datation précise est en cours. On signalera enfin la présence d'un petit ensemble thermal, incomplètement fouillé, au nord-est du mithraeum. Cet édifice a été installé sur un épais radier de graviers, destiné à assainir le marécage sous-jacent. On ignore si ces installations sont liées entre elles d'un point de vue fonctionnel, et jusqu'à quelle époque elles ont pu être en usage. Aucun matériel de l'Antiquité tardive n'a toutefois été mis en évidence dans cette zone.

Au total, les deux grandes phases chronologiques qui ont été distinguées montrent assez bien l'évolution qu'a connue ce secteur : à une série d'installations liées à la présence militaire, puis, peut-être, à un premier établissement civil mal connu, le long de la voie nord-est - sud-est, conformément à un processus bien connu sur le limes (Sommer, 1988, 1994a et b, 1995, 1999), fait suite, dans le courant du $\mathrm{II}^{\mathrm{e}} \mathrm{s}$., une série de grands édifices implantés par rapport à un nouvel axe de circulation est-ouest. Les plus importants, au nord de cette zone, n'ont pas été fouillés. Entre ces grands bâtiments publics (thermes, mansio, magasins, temple ?) apparaissent des structures d'habitat plus classique, sur sablières basses et poteaux. On a un peu de peine à imaginer que ce quartier, périphérique par rapport au cœur de l'agglomération civile, et implanté à proximité du Rhin, n'ait pas été lié au passage du fleuve.

M. R., M. J., C. S. et P.-A. S.

\section{UNE ZONE D'HABITAT AU PIED DE LA BUTTE D'ALTKIRCH}

Au flanc oriental de la butte d'Altkirch a été explorée une zone de confluent entre deux petits cours d'eau actifs dans l'Antiquité ${ }^{10}$. À l'ouest, le Riedgraben marque le pied de la terrasse fluviale; au nord, un petit chenal, de direction générale est-ouest, est pour l'instant assez mal connu. Les deux cours d'eau ont été canalisés et bordés par des quais. Au sud, une voie qui organise l'espace et l'habitat pendant tout le HautEmpire a été mise au jour (fig. 21).

\section{Les chenaux}

Vers l'ouest, le cours d'eau naturel semble avoir été aménagé à l'aide de fagots de branchages et de claies tressées autour de pieux enfoncés dans la vase. Sa rive orientale est marquée par une série de pilotis dans lesquels ont été parfois calés des bois remployés. Cet aménagement est désormais daté de 19 apr. J.-C., grâce à une série homogène de dates dendrochronologiques (datation O. Girardclos, V. Bouillot, Cèdre). Ensuite se met en place un quai, dont l'orientation nord-sud diverge ici de celle du chenal naturel (fig. 22). Ce quai est

10. La fouille de ce secteur a été assurée par M. Reddé, B. Gissinger, J. Pellissier et C. Petit. 


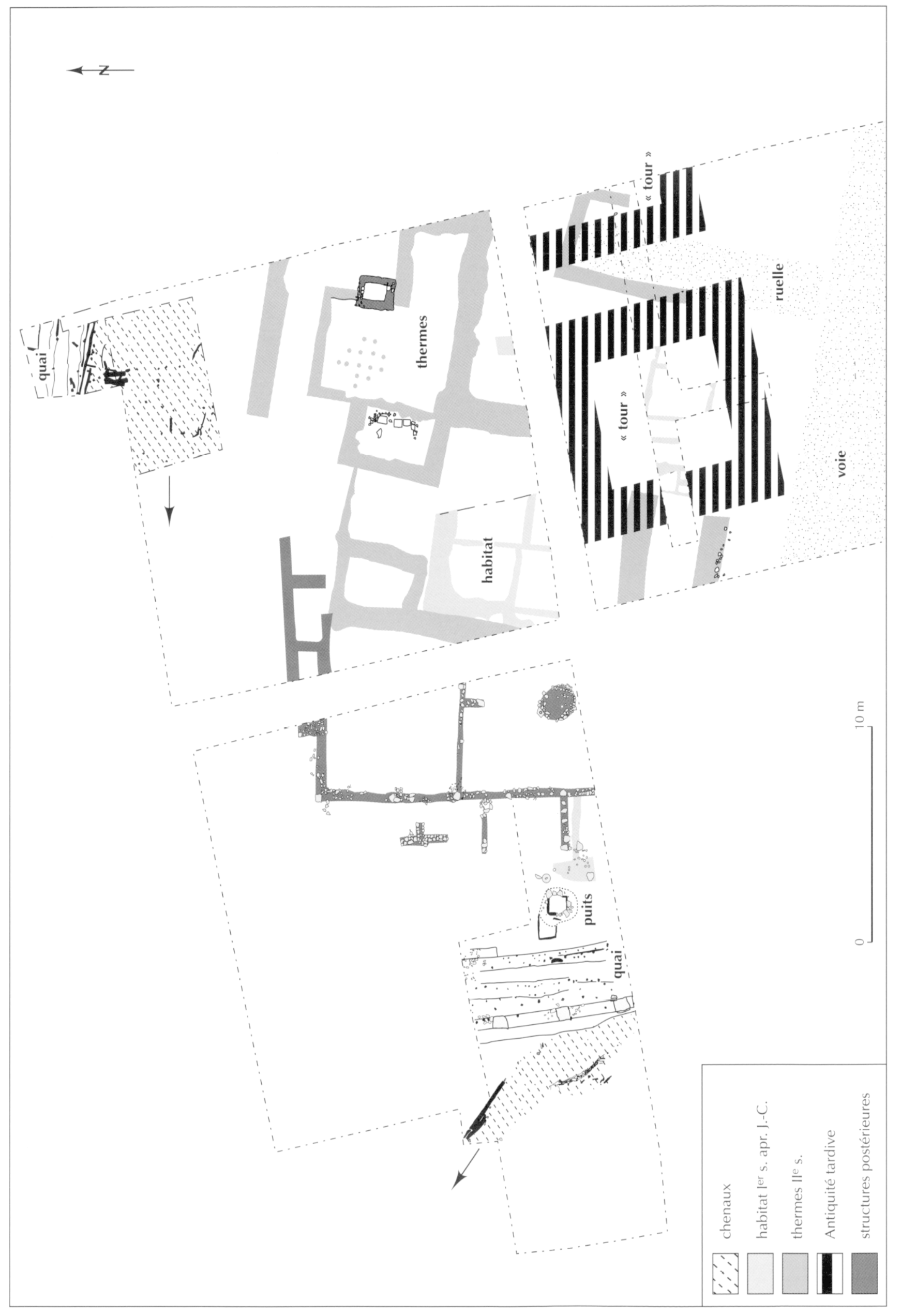

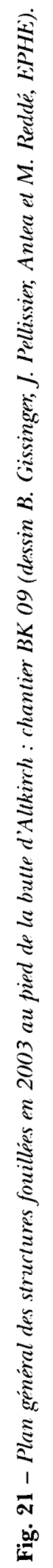


constitué d'une série de palplanches parallèles de résineux qui coffrent un remblai de gravier très riche en céramique. Traversant ce remblai, une forêt de pilotis en chêne supportait probablement une plate-forme qui a disparu, mais servait sans doute de voirie, le long du Riedgraben. Cette chaussée apparait très clairement sur l'image géophysique. L'étude céramologique préliminaire permet de dater cet aménagement entre la fin du I ${ }^{\mathrm{er}} \mathrm{s}$. et le milieu du $\mathrm{II}^{\mathrm{e}}$ s., ce que confirment de nombreuses dates dendrochronologiques. Des réfections ont eu lieu jusqu'en 180. Dans un dernier temps, une série de plots de pierre a sans doute servi à renforcer et stabiliser le système.

Le chenal nord semble avoir été aménagé selon des principes identiques. Son quai, au nord, est lui aussi coffré à l'aide de palplanches. Une série de pieux traverse le fond du chenal, formant probablement une petite passerelle.

\section{L'habitat du $\mathrm{I}^{\mathrm{er}}$ s. apr. J.-C.}

I.'habitat est structuré par une voie de gravier damé (fig. 21). Six couches au moins ont été identifiées, qui ćlargissent progressivement la chaussée. Perpendiculairement à cette dernière, une ruelle nord-sud a été reconnue.

la fouille a mis en évidence, par sondages, une série de sablières en bois reposant parfois sur un lit de gravier; cet ensemble est orthonormé à la voirie, sans que nous soyons en mesure de déterminer de façon précise un parcellaire, car les murs mitoyens des blocs n'ont pas été reconnus. L'ensemble a subi un incendie assez violent, qui a calciné les poutres, parfois effondrées sur place, et a conduit à un nivellement général de la zone. On observe en effet, sur toute l'étendue du chantier, une couche de déblais de constructions à pans de bois, orange, brûlée, d'épaisseur régulière. Un tel phénomène était déjà apparu dans les niveaux sous-jacents de la forteresse valentinienne, à $200 \mathrm{~m}$ de là, ce qui semble indiquer un feu assez. étendu, si ces deux épisodes sont strictement contemporains. Vers l'ouest, du côté du chenal, deux puits ont été reconnus.

Le plan général et la nature des structures plaident incontestablement pour un habitat à colombages, globalement datable de la seconde moitié du $\mathrm{I}^{\mathrm{er}}$ s. apr. J.-C., d'après le matériel en place. L'organisation de l'espace, orthonormé à la voirie, invite à considérer l'hypothèse d'un parcellaire, que d'autres études ont récemment mis en évidence dans les agglomérations du limes de Germanie supérieure (Sommer, 1988, $1997,1998)$.

\section{Les thermes du $\mathrm{II}^{\mathrm{e}}$ s. apr. J.-C.}

Après cet incendie, l'espace est restructuré, sans doute au début du II ${ }^{\mathrm{e}}$ s., si l'on en croit une monnaie neuve de Trajan qui reposait sur l'un des derniers niveaux de l'état précédent (inv. 03-09-00-04 ; RIC, II, 626, frappée en 112-114). L'habitat privé fait place à un ensemble thermal, sans doute public à en juger par sa taille. Fait remarquable, l'axe des constructions continue de respecter les orientations précédentes, signe que la voirie précédente continue de desservir la zone. Le plan de cet ensemble thermal, dont environ $400 \mathrm{~m}^{2}$ sont connus, est incomplet, et d'ailleurs un peu incertain dans le détail, compte tenu des spoliations et destructions qui l'ont affecté après son abandon. On distingue assez bien un caldarium en équerre au nord, dont la suspensura était encore partiellement préservée.

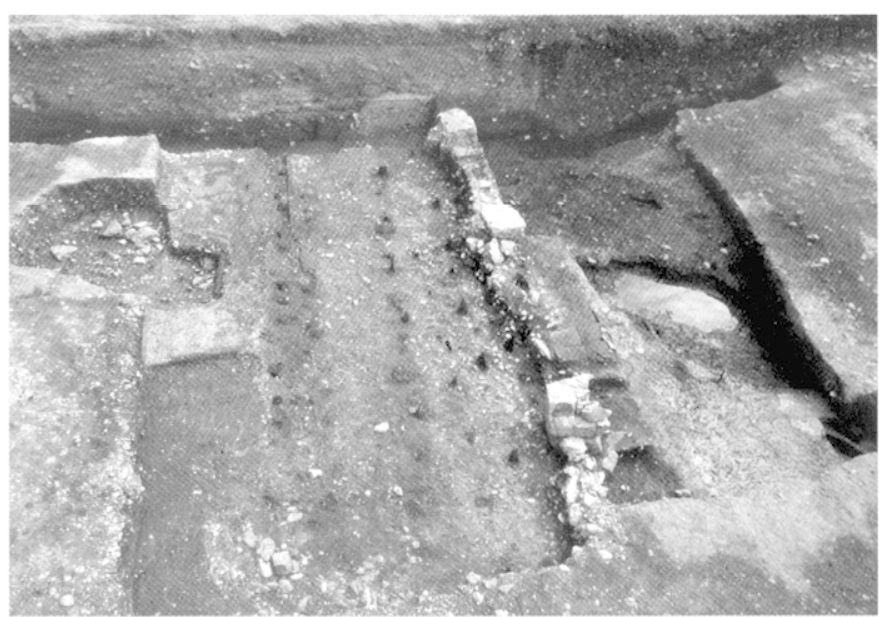

Fig. 22 - Aménagements de quai en bordure du Riedgraben (cliché M. Reddé, EPHE).

Le foyer, situé immédiatement à l'ouest, n'était plus reconnaissable que grâce au seuil de la chambre de chauffe. Plus à l'ouest et au sud-ouest existaient sans doute des espaces de service, et les autres salles thermales proprement dites devaient se développer vers l'est et le sud-est. Le matériel mis au jour ne permet pas de dire précisément jusqu'à quand cet ensemble a fonctionné. Une utilisation dans le premier quart du $\mathrm{III}^{\circ} \mathrm{s}$. est probable. Après cette date, on constate la présence, all-dessus de ces structures en ruines, d'une couche générale de gravier qui a servi de préparation aux constructions de l'époque suivante. Mais l'absence quasi complète de matériel dans ce niveau ne permet pas d'en préciser la chronologie.

\section{Les structures tardo-romaines}

C'est pendant l'Antiquité tardive que sont construites deux substructions carrées, espacées de $2,50 \mathrm{~m}$, construites en blocs de basalte. Leur orientation est identique, mais diffère des bâtiments des phases antérieures.

Il ne reste de la maçonnerie que l'assise inférieure des fondations et quelques noyaux de blocage, pour des murs épais de $1,75 \mathrm{~m}$. Aucun pieu de bois n'a été retrouvé en dessous, contrairement à ce qui a été observé sous la forteresse valentinienne (voir infra, p. 246). Si l'on en croit la prospection géomagnétique, les bâtiments forment des sortes de " tours " carrées, de $9 \mathrm{~m}$ de côté environ. La découverte en prospection de nombreuses monnaies du IV $\mathrm{V}^{\mathrm{e}}$ s. ne laisse pas planer de doute sur la datation générale, mais on ne peut guère être plus précis. On ne saurait, de toute façon, décider si ces " tours ", ou l'une des deux seulement, a pu être en usage en même temps que la forteresse de Valentinien, avant celle-ci, ou après elle. D'un strict point de vue fonctionnel, on voit mal à quoi une tour isolée, située à moins de $200 \mathrm{~m}$ en contrebas de la forteresse, pourrait servir. L'existence simultanée de ces différents édifices paraît donc peu probable.

\section{Les structures postantiques}

La vie du secteur ne s'arrête pas avec cette période. Le site est en effet réoccupé par une série de murs étroits, en blocs de basalte à sec, qui font un large usage des remplois in situ, et qui 


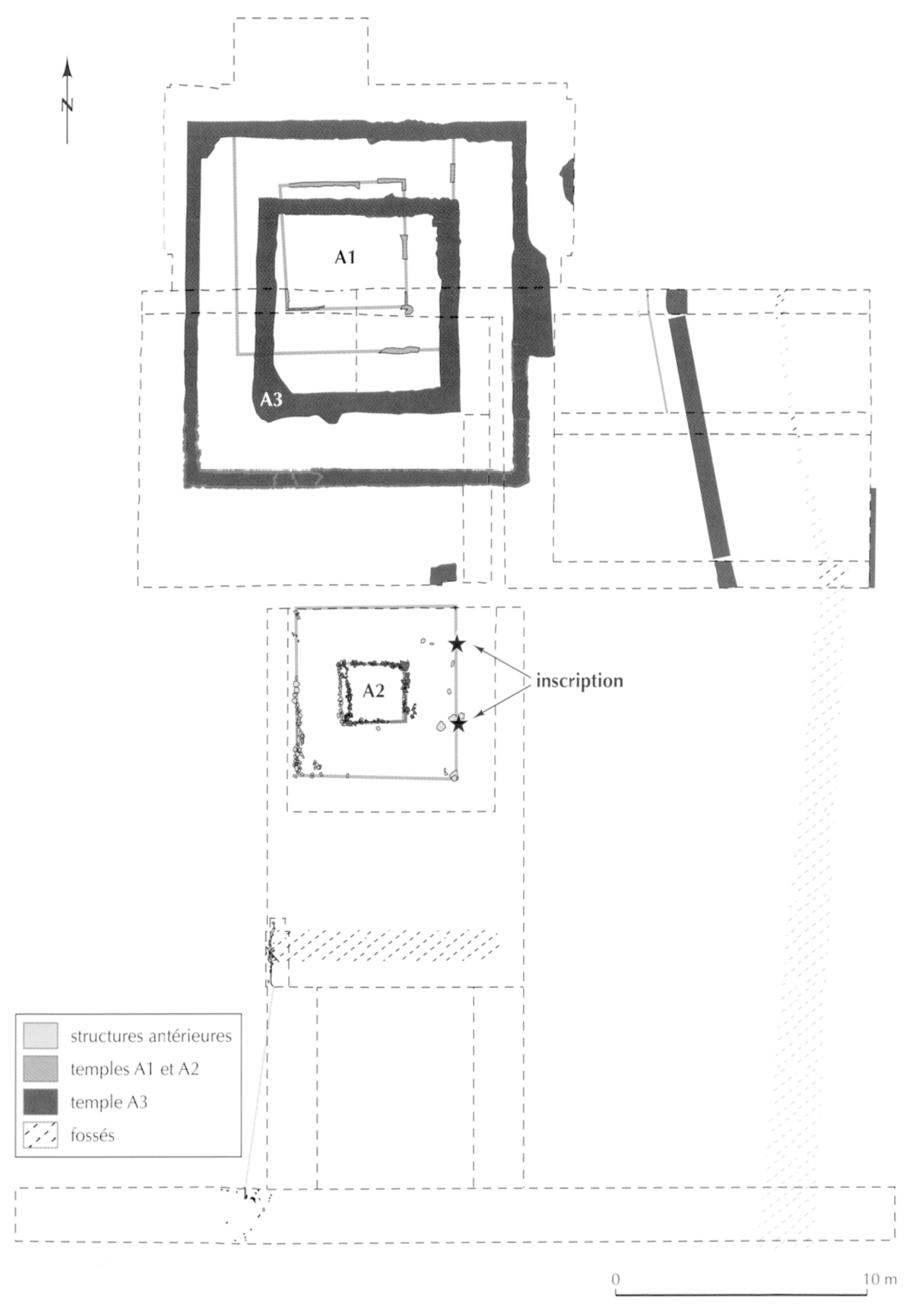

Fig. 23 - Plan général du temple A : chantier BK 03-(15 (dessin (.. Schucany, univ. de Bâle).

délimitent une série de pièces avec un sol épais de gravier bien damé. Ces constructions, très proches de la surface du sol actuel, et fortement ruinées, ne sont pas clairement datées en l'absence de tout matériel scellé. Elles ont à leur tour été réoc- cupées, notamment par une série de tombes, médiévales ou modernes, dont la datation précise est en cours.

I a fouille de cette zone permet ainsi de suive de manière assez complète les différents états du développement 


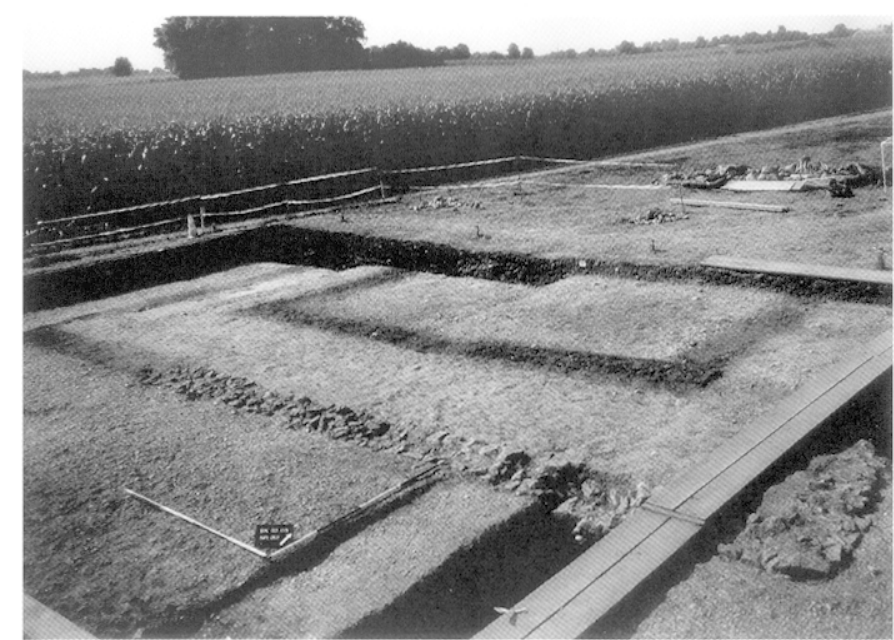

Fig. 24 - Le temple A3, vue du sud-est (clichéS. Straumann).

d'Oedenburg : aménagement des espaces naturels et des voies d'eau, mise en place d'une voirie urbaine et d'un parcellaire, évolution de l'habitat, devenir du site après l'Antiquité.

M. R., M. J., B. G. et J. P.

\section{LE SECTEUR DES TEMPLES}

Les prospections géomagnétiques ont révélé des plans de temples de forme indigène où du mobilier riche, récolté en surface, permettait a priori d'en supposer l'existence. Ils font partie soit d'une unité plus grande, soit d'une aire sacrée. Son extension est comparable à celle de Cambodunum à Kempten, de Mogontiacum à Mayence ou d'Augusta Treverorum à Trèves, alors que les temples indigènes connus à ce jour dans le caput de la colonia Augusta Raurica à Augst et Kaiseraugst sont de dimensions nettement inférieures.

On peut reconnaître un ensemble de trois temples qui se trouvent dans des enclos, séparés des autres, mais qui semblent cependant former une unité. Le temple occidental a été fouillé par l'université de Bâle ${ }^{11}$ (fig. 23). Les affleurements géologiques et topographiques indiquent que la zone du temple longe un ancien bras du Rhin, d'axe nord-sud. À l'est, il est délimité par un banc de gravier bien marqué, orienté lui aussi du nord au sud, où ont été installés les deux autres temples.

\section{Le I ${ }^{\text {er }}$ s. apr. J.-C.}

Dans la zone marécageuse, des concentrations de mobilier archéologique, les restes d'un arbre abattu, une rangée de pieux et de petits canaux de drainage témoignent d'activités anthropiques dès l'hiver 3-4 apr. J.-C. (datation O. Girardclos, V. Bouillot, Cèdre). La céramique date de la première moitié du $\mathrm{I}^{\mathrm{er}}$ s. apr. J.-C., les pièces les plus récentes ont été produites après le milieu du $\mathrm{I}^{\mathrm{er}} \mathrm{s}$. On ne saurait exclure que ces structures et ce mobilier présentent des liens avec d'éventuelles construc-

11. La fouille de ce secteur a été dirigée par C. Schucany et P.-A. Schwarz. tions installées sur le banc de gravier avant l'édification des deux temples à l'est.

L'assainissement de la zone palustre fut entrepris vers 70 apr. J.-C. Après la mise en place d'un niveau de branchages, on y étala une couche de gravier sur laquelle furent édifiés les deux premiers temples. Celui du nord (A1), d'une surperficie de près de $8 \mathrm{~m} \times 9 \mathrm{~m}$, disposait d'une grande cella ainsi que d'un portique. Les parois étaient en torchis enduit de mortier de chaux blanc. Des restes de peinture pompéienne rouge, jaune, verte, bleue et noire indiquent qu'elles étaient ornées de champs et de bandes colorés, ainsi que de motifs de guirlandes.

Le temple sud (A2) fut érigé parallèlement ou à une époque légèrement plus tardive. Contrairement à l'édifice nord, ses sablières basses reposent sur une couronne de pierres. Une interprétation comme temple à proprement parler demeure incertaine. La couronne de pierres intérieure pourrait tout aussi bien se rattacher à une chapelle, alors que celle située à l'extérieur aurait pu soutenir un mur d'enceinte. Les parties en élévation se composaient vraisemblablement de parois à colombage.

\section{Le II ${ }^{\mathrm{e}}$ s. apr. J.-C.}

Au début du $\mathrm{II}^{\mathrm{e}}$ s., on démantela le temple nord pour ériger un temple plus grand A.3 (fig. 23 et 24). Sa cella en maçonnerie, probablement haute de deux étages, mesurait environ $8 \mathrm{~m}$ x $8 \mathrm{~m}$. Tout comme le portique, elle était recouverte de tuiles. Le portique était vraisemblablement muni d'un dallage de pierres reposant sur un lit de sable, dégagé sur une grande surface lors de la fouille. Le taux élevé de précipitations de phosphate dans ce lit de sable pourrait indiquer que, sous le portique, on gardait ou abattait des animaux destinés aux rites sacrificiels.

Le temple sud ne fut très vraisemblablement démoli qu'une fois l'édification du nouveau temple nord achevée. Les fondations de la cella furent renforcées de blocs de basalte. Elles servirent par la suite de substruction à un autel votif érigé sur de grands blocs. Ce sont les deux fragments contigus d'une inscription votive qui fournissent des indices pour sa reconstitution (fig. 25) ; ils ont été mis au jour à proximité immédiate des

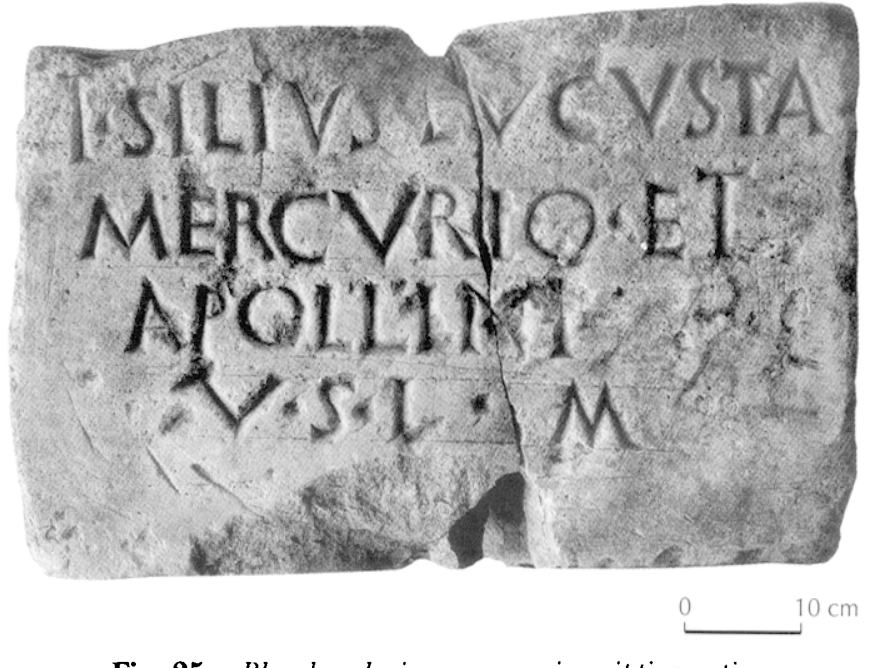

Fig. 25 - Bloc de calcriore aver une inscription votive. (cliché S. Straumann). 
fondations. Le texte de l'inscription votive, entièrement conservé, est le suivant: TI(berius) SILIVS LVCVSTA / MERCVRIO ET / APOLI.INI / V(otum) S(olvit) L.(ibens) M(crito), c'est-à-dire : “ À Mercure et Apollon, de la part de Tiberius Silius Lucusta, qui s'est volontiers acquitté de son veu " ${ }^{12}$. Grâce à l'évocation des deux divinités, on sait que le temple était dédié à Apollon et à Mercure.

La proportion exceptionnelle de monnaies couvrant la période allant du début du $\mathrm{I}^{\mathrm{er}} \mathrm{s}$. au milieu du IV s. reflète sans doute la pratique du dépôt de monnaies, durant l'Antiquité, dans la zonc du temenos du temple. En effet, dans le niveau de circulation du temple et de l'autel aussi bien que dans les niveaux archéologiques situés à l'est du temple, on ne relève qu'une faible proportion de céramiques et de matéricl faunique. Selon le mobilier datable, ce temple fut fréquenté durant tout le $\mathrm{II}^{\mathrm{e}} \mathrm{s}$. et au moins la première moitié du $\mathrm{III}^{\mathrm{e}} \mathrm{s}$.

\section{Les activités postantiques}

L.e démantèlement des murs dans l'intention de récupérer des matériaux de construction se fit sans doute en plusieurs ćtapes, et sur un temps asse\% long. Les dimensions, les méthodes de construction et d'autres indices encore permettent de supposer que l'autel fut démantelé dès le Bas-Empire. C'est aussi à cette époque que les éléments en élévation du dernier temple, peutêtre en ruines ou nécessitant une rénovation, furent sans doute cux aussi pillés. Pour le démantèlement des vestiges demeurés in situ, sous le niveau de circulation le plus récent, on peut postuler une intervention tardive, au XI'II" ou au XVIII" s. (voir annexe, infra, p. 268-269).

C. S. et P.-A. S.

\section{L'ANTIQUITÉ TARDIVE AUTOUR DES BUTTES DE WESTERGASS ET D'ALTKIRCH}

Depuis longtemps, sous l'impulsion de chercheurs renommés (R. Nierhaus, J. Garbsch, G. Fingerlin, H. Bender, R. Swoboda et H. Steuer), on a cherché, notamment en Allemagne, à étudier la rive droite du Rhin, afin de préciser l'histoire du Bas-Empire et du haut Moyen Âge dans les régions dı Rhin supérieur, en particulier dans la zone du Kaiserstuhl (fig. 26). C'est R. Christlein qui, pour la première fois, a suggéré la présence possible d'une fortification romaine du Bas-Empire à Oedenburg (Christlein, 1974). Malgré tous ces efforts, on ne disposait, jusqu'à une période récente, que de peu de données sur ce site.

L'importance de la situation géographique d'Oedenburg n’a cessé de croître depuis le début du Haut-Empire jusqu'à la fin de l'Antiquité, avec cependant cette grande différence qu'à partir du III" s. apr. J.-C., les régions situées sur la rive droite du Rhin, particulièrement celles à l'est de la Forêt-Noire, peuplées d'Alamans (Alamannia), n'étaient plus sous l'administration directe de Rome. En ce qui concerne les voies de communication au niveau de Breisach-Oedenburg, rien n'a fondamentalement changé après 260, lors de l'abandon par Rome de certaines régions situées sur la rive droite du Rhin. Une borne

12. La lecture Ti(berius) ne peut être totalement exclue, en raison de la hauteur de la haste verticale du T, qui dépasse la barre transversale.

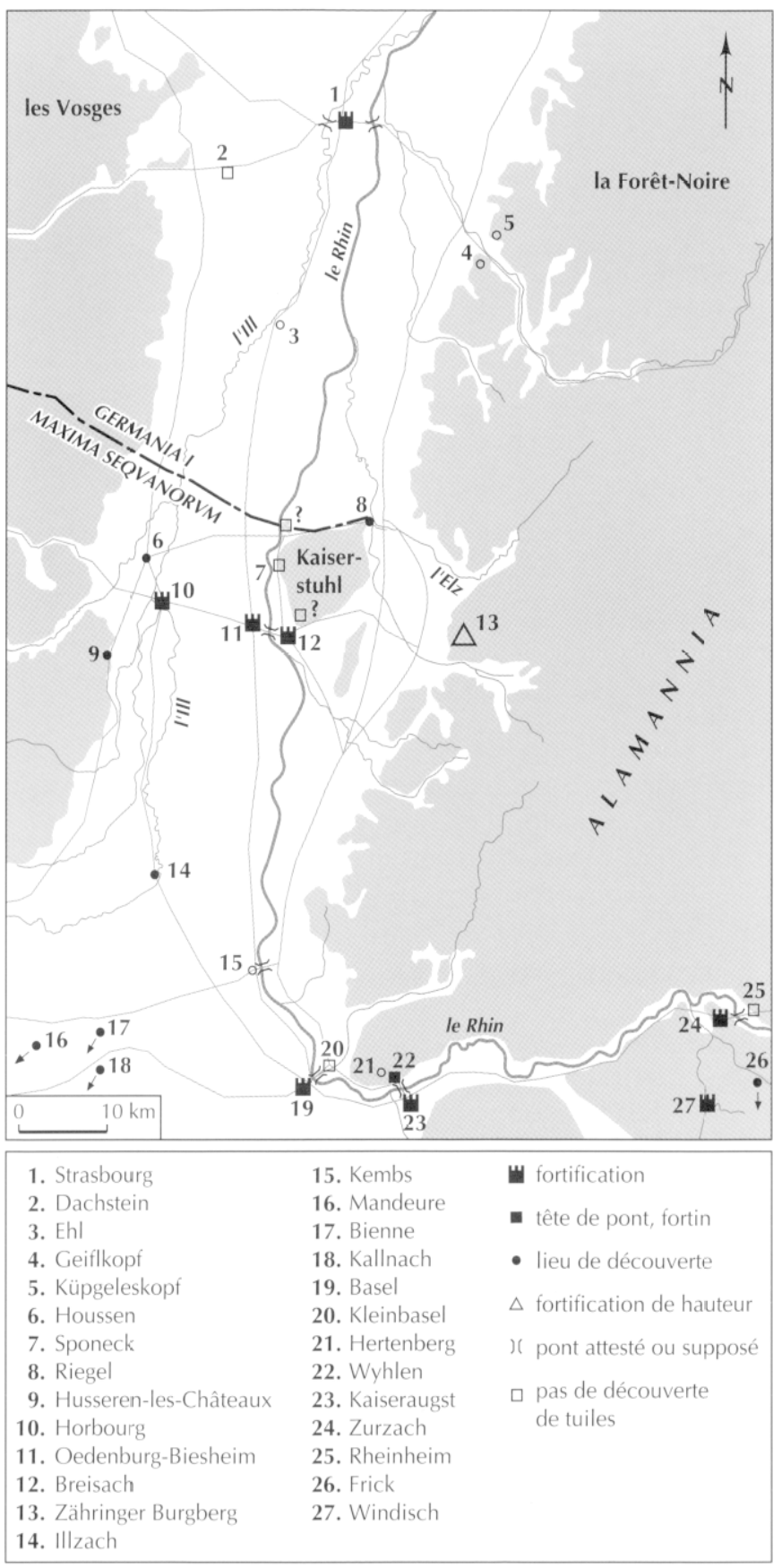

Fig. 26 - La région du Rhin supérieur au Bas-Empire et la diffusion des tuiles de la Legio I Martia (dessin S. Feldhusen).

milliaire de Postumus montre que la via publica, c'est-à-dire la liaison sud-nord, par terre et par cau, était encore en usage (Nuber, 2000b). Il en allait de même pour le franchissement du Rhin.

\section{LA TOPOGRAPHIE D'OEDENBURG DANS L'ANTIQUITÉ TARDIVE}

Les lieux-dits Westergass et Altkirch ressemblent actuellement à de faibles collines. Celles-ci ont cependant une origine différente. Alors que le sous-sol du Westergass (alt. 188,80 m 
NGF) est formé vers l'est par un banc de gravier naturel recouvert, à l'ouest, d'alluvions sableuses, la butte d'Altkirch (alt. 192,10 m NGF) est le résultat de l'occupation du site pendant des centaines d'ammées, à l'époque romaine et postromaine (fig. 5,6 et 27). Tous les indices montrent de manière concordante qu'au-dessus d'une couche d'argile de couleur brun foncé contenant quelques céramiques de l'âge du Bronze, les couches encore conservées de l'occupation et de la voirie datant de l'époque romaine sont empilées sur une hauteur de plus de $1,80 \mathrm{~m}$. La colline était cependant plus élevée à l'époque qu'elle ne l'est aujourd'hui, car les couches les plus récentes (médiérales et romaines tardives) ont été depuis longtemps victimes de l'érosion du sol; celle-ci a en effet tendance à diminuer vers les bords de la butte. Ainsi, dans la partic nord de la fortification (carrés 3-8), les couches supérieures conservées, situćes directement sous la terre arable, datent tout juste encore des environs de 100 apr. J.-C. (sigillée de la Gaule du Sud), alors que la situation change vers le sud (carrés 45-46), où les couches supérieures, fouillées en 2002, présentent encore des sigillées de la Gaule de l'Est (Heiligenberg, Rheinzabern précoce). En revanche, le mobilier plus récent (depuis le milieu du $\mathrm{II}^{\mathrm{e}} \mathrm{s}$. jusqu'au début de l'époque moderne) ne se trouve plus que dans les structures excavées de ces périodes (fosses, tranchées de fondation) ou comme dépôt secondaire dans le remblai des tranchées de fondation pillées, ce qui signific que les couches d'occupation de ces époques manquent.

\section{LE PRAETORIUM DE WESTERGASS}

Le bâtiment principal de $24 \mathrm{~m}$ x $29 \mathrm{~m}$ et le bâtiment thermal de $7 \mathrm{~m} \times 14 \mathrm{~m}$, situé à l'arrière, se dressent sur une légère éminence (fig. 28 et 29), déjà utilisée de façon sporadique au I ${ }^{\mathrm{Cr}}$ s. apr. J.-C. (voir la fibule 99.02.404, fig. 30) comme enclos à bétail ou autre clôture agricole ("Zaungräbchen "). D'après les découvertes monétaires, cet endroit n'a été réoccupé - par les constructions en dur - qu'à partir de l'époque des fils de Constantin I dans les années 330-350. La mise au jour quasi complète des fondations, largement spoliées, a révélé des murs partiellement construits avec des pierres de remploi, qui déterminent un plan clairement structuré : après avoir traversé un portique orienté vers l'ouest en direction de la route, on atteignait une cour intérieure ouverte. Celle-ci était entourée sur trois côtés d'une galerie de pièces à plusieurs étages, à l'instar d'un caravansérail. L'approvisionnement en eau n'a pu être éclairci.

La figure 29 montre un essai de reconstitution de l'aspect extérieur du bâtiment. Un parallèle quasi identique est livré par le complexe de bâtiments - hôtellerie et thermes - de Muru-deBangius, en Sardaigne (Zucca, 1992), dont nous connaissons la fonction et le nom (praetorium) grâce à une inscription de l'époque de Septime Sévère. Un bâtiment de même type, légèrement plus grand, est connu, non loin de là, à Kembs $1: 3$ (Fellmann, Wolf, 1993).

13. La comparaison entre ces différents exemples permet didentifier les vestiges du Römerberg, à Francfort-sur-le-Yain (tant discutés dans le passé), comme étant des praetoria romains (Nuber, Seitz, 2001).
La soixantaine de legulae de la Legio I Martia trouvées à cet endroit (fig. 30, $\mathrm{n}^{\mathrm{os}} 11-14$ ) - et sculement à cet endroit n'attestent pas, contraircment à ce qu'on avait supposé à l'origine (Biellmann, 1988 ; Fellmann, 1995 ; Zehner, 1998), la présence d'un grand cascrnement romain, mais prourent simplement que nous sommes en présence d'un bâtiment public. Un autre fragment de tuile estampilléc provenant des thermes nomme un tuilier de l'époque romaine tardive, appelé Amazonius ou Amasonius (fig. 30, n¹0), dont l'atelier était situé dans le nord de la Suisse, aux environs d'Augst. On notera l'absence d'objets militaires dans le proetorium de Westergass. En revanche, des éléments de parure (fibules, épingles, fig. 30) démontrent que des groupes germaniques ont investi les lieux au $\mathrm{v}^{\mathrm{e}}$ s. La dernière phase de l'occupation tardo-antique voit la réutilisation partielle des murs encore debout, tout en étant caractériséc par l'existence d'une architecture en bois (" civilisation du bois").

Pour connaître le sort des bâtiments (praptorium et thermes) après 353-355 apr. J.-C. et savoir s’ils ont été détruits ou s'ils ont continué d'exister, il faut attendre les résultats d'une comparaison minutieuse des corpus de mobilier ${ }^{14}$. Une continuité de l'occupation humaine à cet endroit, jusqu'au Moyen Âge, n'a pu être déterminée.

\section{LA FORTIFICATION D'ALTKIRCH}

\section{Situation, orientation}

La fortification d'Altkirch s'étend du sud au nord entre le rebord oriental de la butte d'où le terrain s'abaisse vers un bras du Rhin (Riedgraben) et la rocade sud-nord, à l'ouest, où elle perce sans doute intentionnellement le bord est de la chaussée (fig. 5 et 27). Nous pensons, bien que le plan de la porte sud n'ait pas encore été identifié définitivement, que l'édifice était orienté vers le sud. L'emplacement de la porte sud, d'où on peut voir la fortification contemporaine situće sur le Münsterberg à Breisach, occupe l'endroit le plus élevé du site (191,76 m NGF). L'espace fortifié s'incline d'ici vers l'ouest (angle sud-ouest à 191,55 m NGF), vers le nord (angle nordouest à 190,49 m) et vers le nord-est jusqu'à l'altitude 189,17 m NGF (angle nord-est), où se situe le point le plus bas.

\section{Dimensions}

Il existe encore un certain degré d'incertitude cn ce qui concerne les dimensions générales de l'édifice (fig. 31). Sauf exception, les mesures n'ont pu être prises qu'au niveau des tranchées de fondation des murs spoliés. Lorsqu'il est question d'épaisseur de murs, il s'agit donc de celles des fondations, car seules celles-ci sont en partie conservées. Trois modules ont été déterminés :

Rappelons que le mot " praetorium ", qui désigne, en contextc militaire. le quartier d'un officier commandant, peut désigner, en contexte civil. et particulièrement à partir du $\mathrm{III}^{\circ}$ s., un relais de peste (voir notamment Levi, Levi, 1967 ; Zucca, 1992 ; Fellmann, Wolf, 1993).

14. Les monnaies ( $H$. L. Nuber) et les objets de bromz (M. Zagermann) de Westergass ont éte identifiés ainsi que la céramique, qui a été entièrement étudiée par G. Meyer : tous appartiennent à l'université de Fribourg-en-Brisgau. 
4

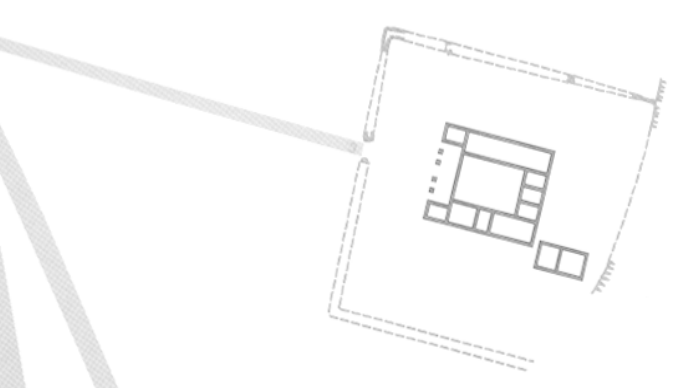

Oedenburg-Westergass
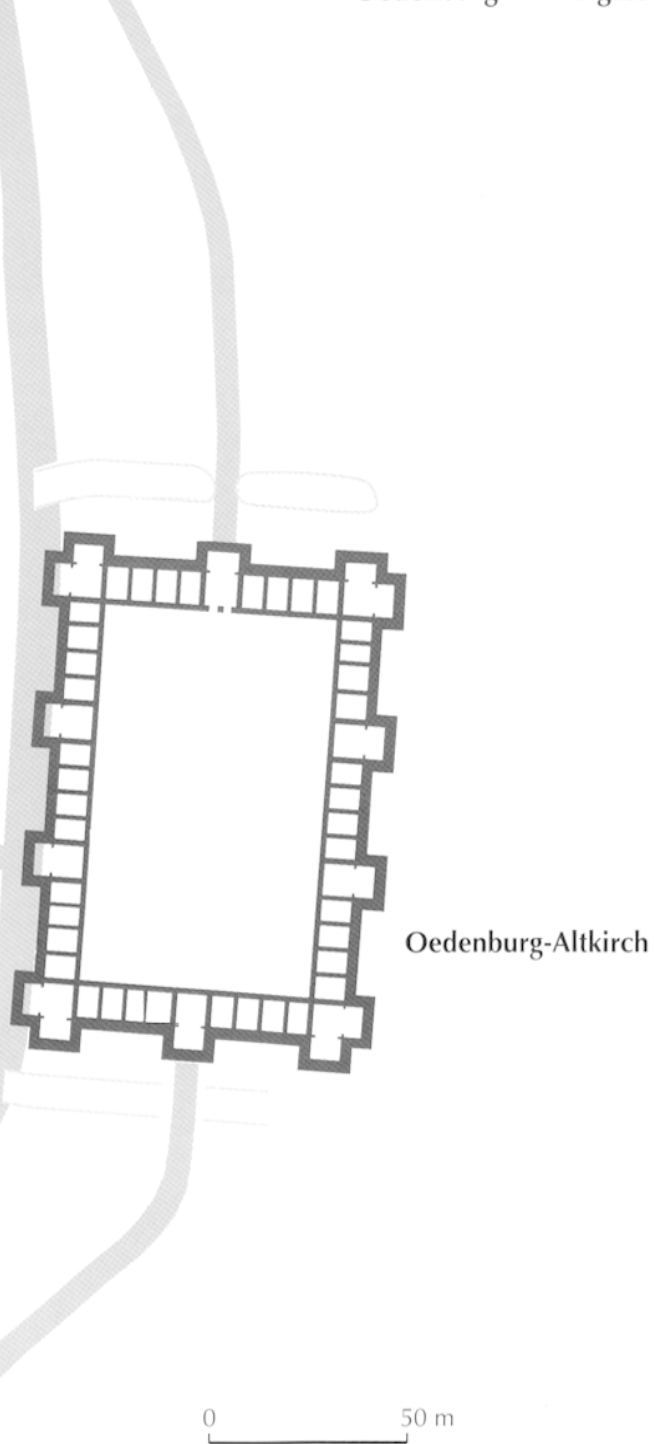

Fig. 27 - Situation topographique des constructions du Bas-Limpire et du réseau routier d'Oedenburg-Allkirch el Oedenburg-Westergass (dessin W. Derpmann-Wirsing). 


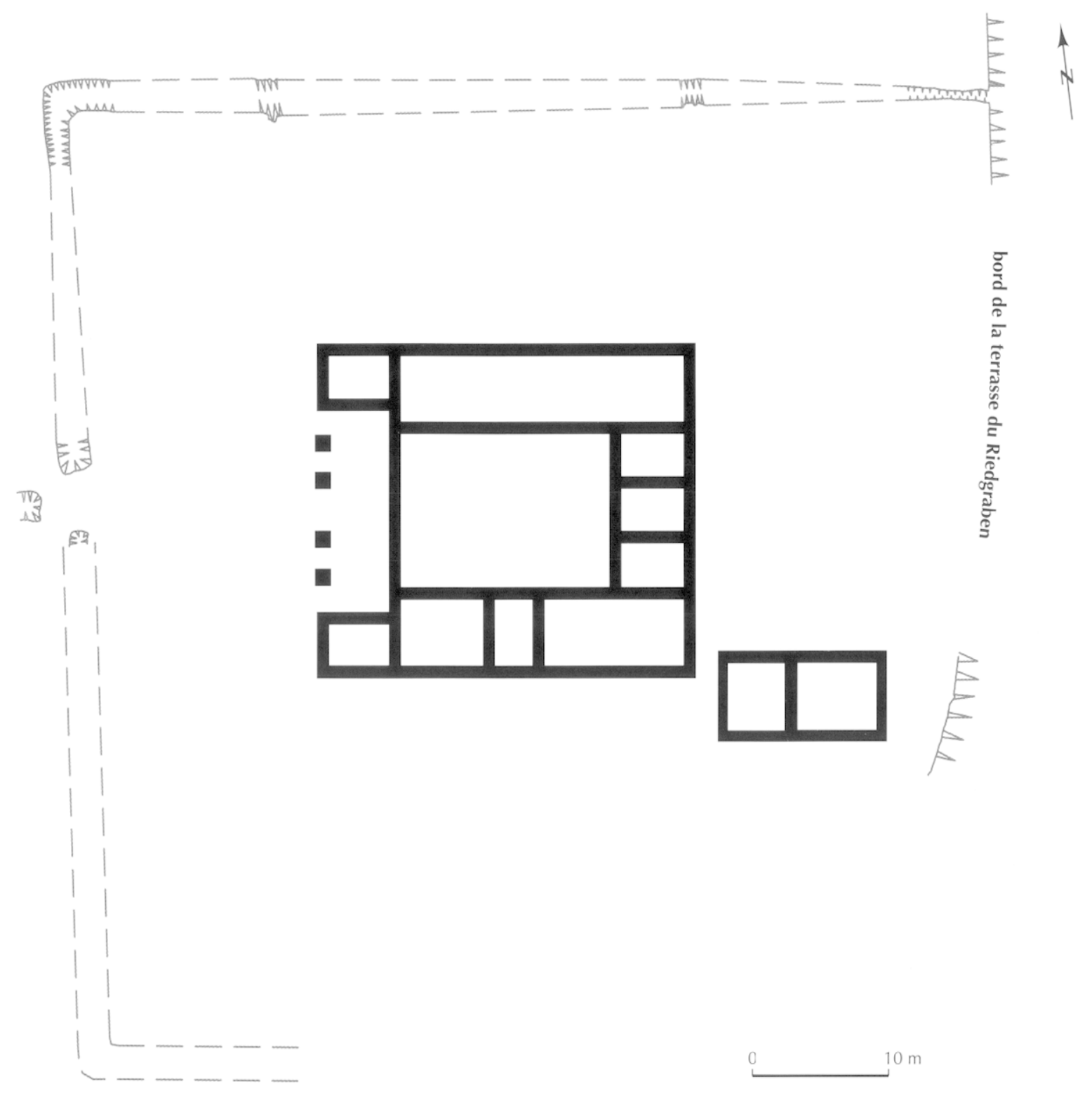

Fig. 28 - Plan général d'Oedenburg-Westergass (dessin S. Feldhusen).

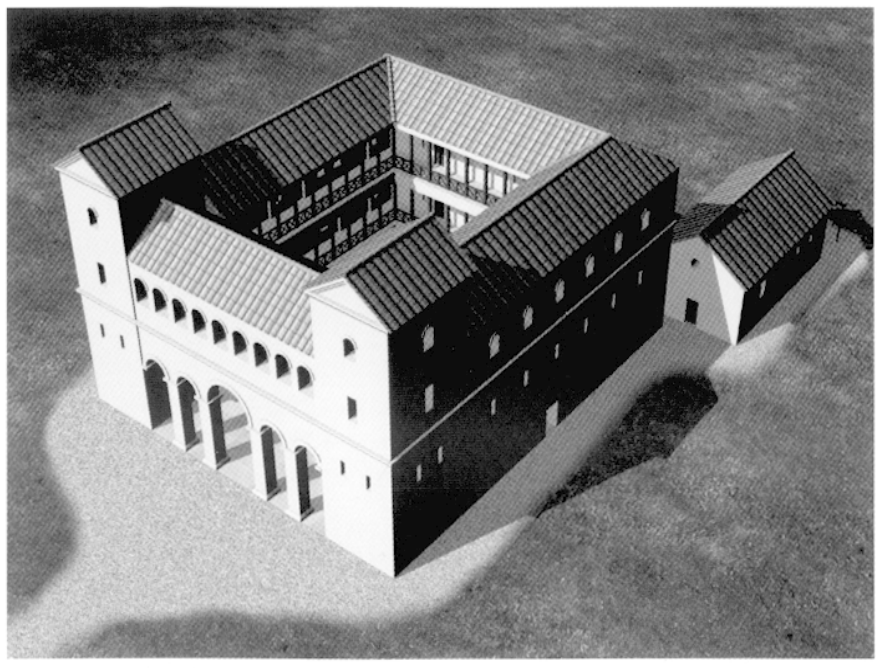

Fig. 29 - Oedenburg-Westergass : reconstitution du praetorium avec ses thermes, vue depuis le sud-ouest (dessin S. Feldhusen). 

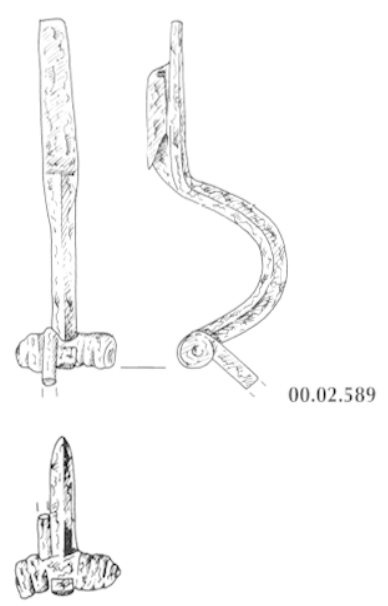

1

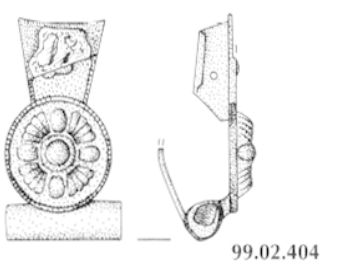

5

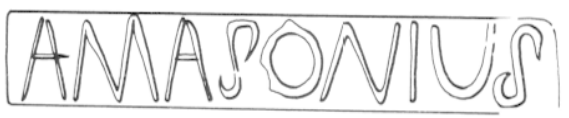

10

00.02 .601

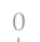

0

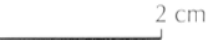

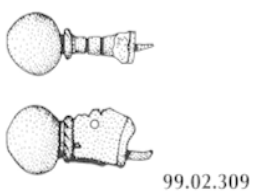

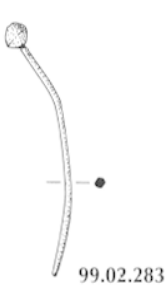

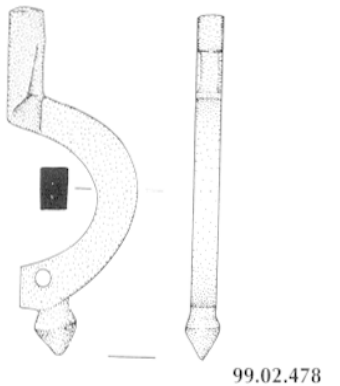

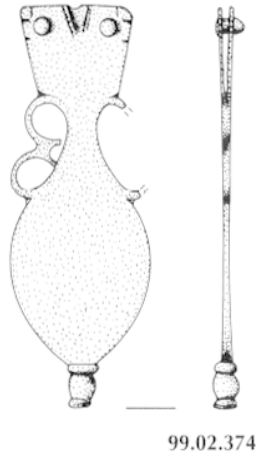

4

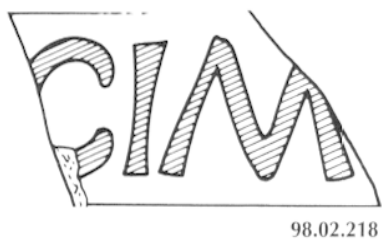

11

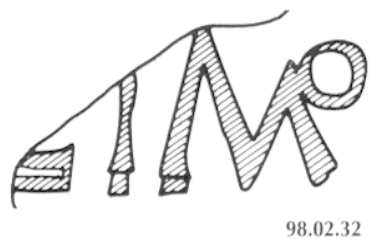

12

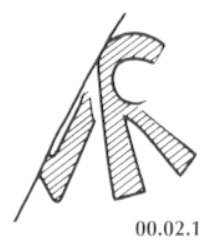

13

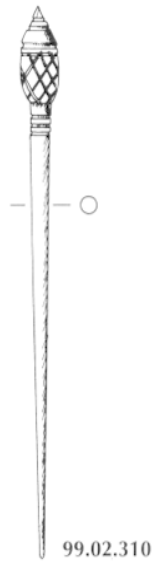

8

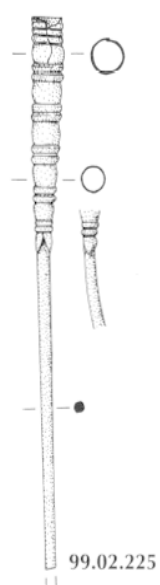

9

Fig. 30 - Eléments de parure et marques des briques d'Oedenburg-Westergass (dessin A. Schwellnus).

- $3 \mathrm{~m}$ pour le mur extéricur de la fortification ;

- 1,20 m pour les murs de refend des cascrnements ;

- 1,50 m pour le mur intérieur du casernement et les murs de refend à l'intéricur des bastions.

Grâce à la découverte d'un morceau de mur maçonné en tuiles (2002) US 955), nous savons que ce n'est pas le pied romain de $0,295 \mathrm{~m}$ qui a été utilisé lors de la construction de la fortification, mais le pied germanique de Drusus $(0,332 \mathrm{~m})$.

La plus grande largeur de la fortification - mesuréc entre les parois extérieures des tranchées de fondation pillées des bastions est et ouest, situćs le plus au nord - attcint $92,95 \mathrm{~m}$. La plus grande longueur - mesuréc entre les parois extérieures des tranchées de fondation pillées des bastions sud et nord, situés le plus à l'ouest - atteint 125,77 m. Ia longucur extérieure des bastions varie entre $13,44 \mathrm{~m}$ et 13,96 m, et leur largeur extérieure mesure 4,90 m ; sur le front ouest, la longueur extérieure des bastions diminue du nord au sud de 13,89 $\mathrm{m}$ à 13,44 $\mathrm{m}$. Les casernements mesurent $\mathrm{en}$ moyenne 7,50 $\mathrm{m}$ de long et $4,30 \mathrm{~m}$ à $5,45 \mathrm{~m}$ de large (dimensions internes). Les dimensions de la cour intéricure sont de $58 \mathrm{~m}$ $x$ 89,6 m. I a surface de l'ensemble est de 1,06 ha.

\section{Structure du plan et technique de construction}

Indépendamment de la question relative à la présence d'une construction intérieure centrale, question qui n'est pas cncore définitivement réglée, la fortification se distingue par son concept architectural qui repose sur un plan clairement 


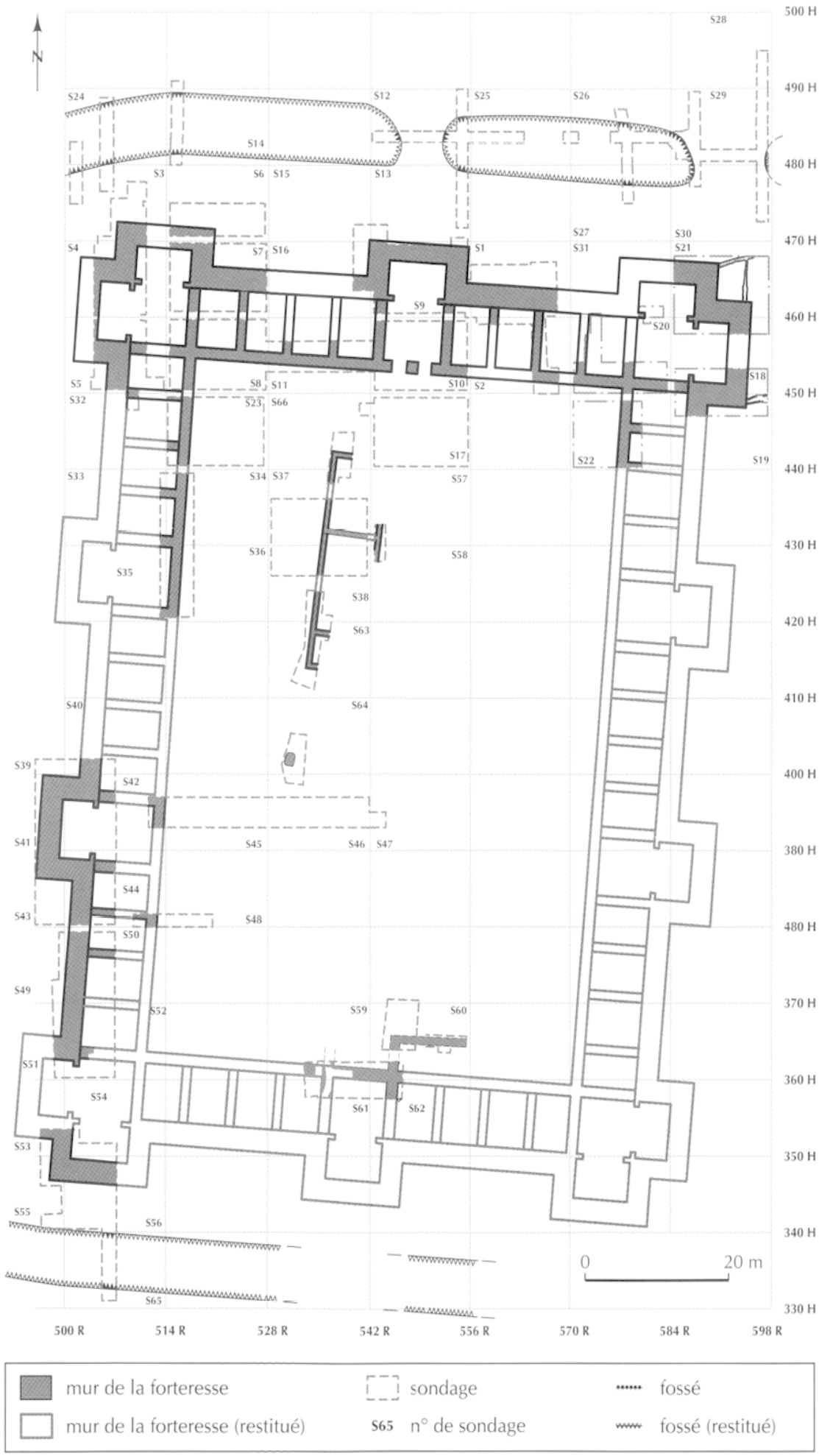

Fig. 31 - Plan général avec implantation des carrés à Oedenburg-Altkirch : état en 2003 (dessin W. Derpmann-Wirsing).

structuré (fig. 31). Sans prendre en compte les côtés est et ouest, où la question reste encore ouverte, nous avons pu mettre en évidence, au sud et au nord, des fossés à fond plat de 2 à $3 \mathrm{~m}$ de profondeur et de 6 à $8 \mathrm{~m}$ de largeur. Le fossé septentrional est interrompu devant la porte nord par un terre-plein. L'épaisseur d'environ $3 \mathrm{~m}$ du mur d'enceinte (mur extérieur) est caractéristique de l'époque (Anthes, 1918). Du côté intérieur s'adossaient, le long des quatres côtés, des casernements ressemblant à des casemates, comme on en connait à Alzey, phase I (Oldenstein, 1994) ou à Altrip (Schnurbein, Köhler, 1989). Nous avons cherché vainement à l'ouest, au nord et à l'est un portique comme celui qui existe à Altrip. L'aménagement ressemble visiblement davantage à celui d'Alzey, où les traces d'un portique font également défaut. 
C'est seulement en 2003 que nous avons pu mettre au jour des traces qui indiquent, au moins à la porte sud, l'existence d'un mur de fondation d'un portique.
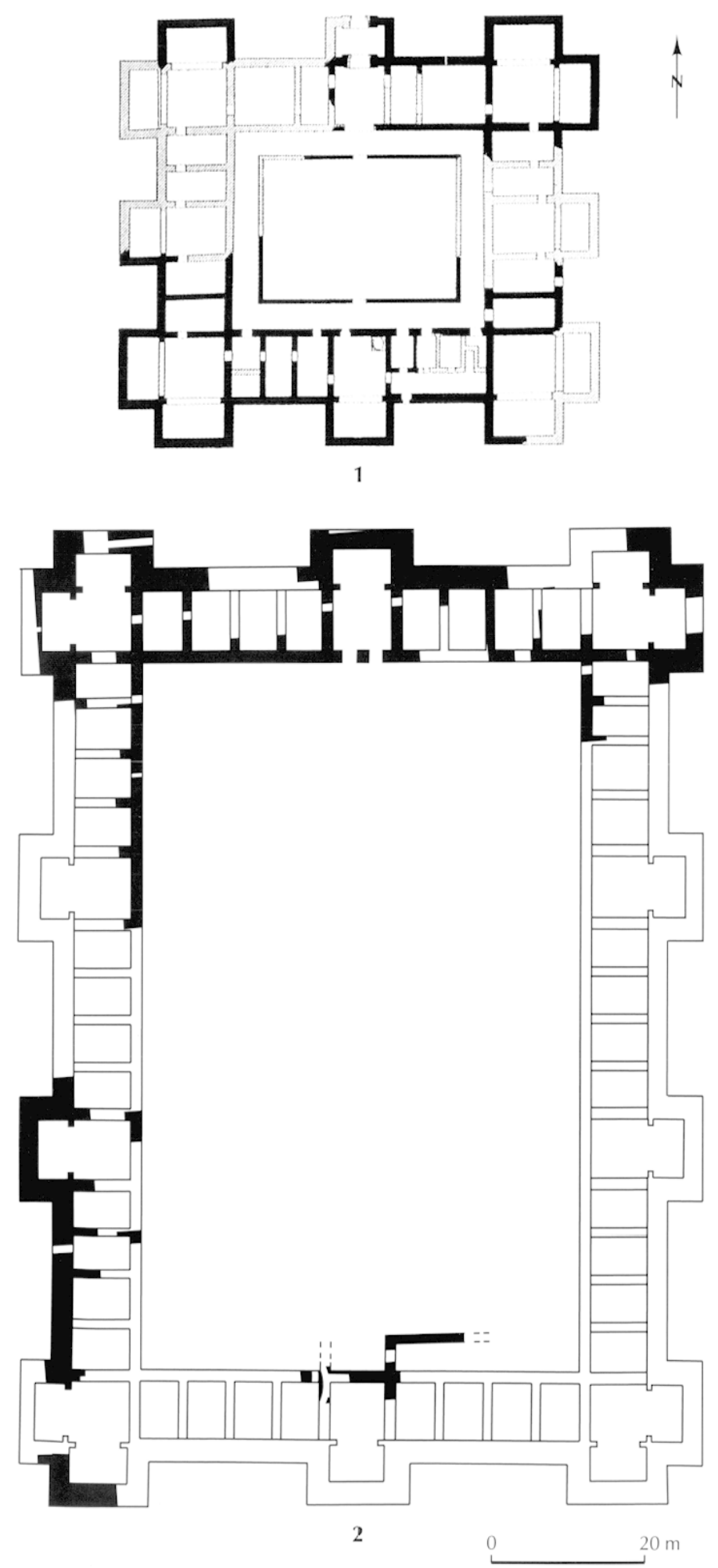

Fig. 32 - Comparaison des dimensions des plans : 1, Trier-Pfalzel; 2, Oedenburg-Altkirch (dessin S. Feldhusen et S. Berg, univ. de Fribourg-en-Brisgau).
Malgré d'importantes perturbations, la porte nord a pu être identifiée, grâce à l'interruption du fossé et à la présence d'une voie d'accès, en raison aussi de l'interruption du mur intérieur. Les casernements de l'aile nord forment deux séries de quatre pièces, de part et d'autre de l'espace plus grand qui correspond au bastion de la porte nord. Sur les ailes occidentale et orientale, nous retrouvons la même organisation, avec trois séries de quatre casemates, séparées par des pièces plus grandes au niveau des bastions. Or, dans la mesure où le mur intérieur de l'aile ouest n'y est pas interrompu, nous ne savons pas encore l'existence et le nombre des portes éventuelles vers l'ouest, c'est-à-dire vers la via publica. Le troisième bastion de l'aile occidentale a pu avoir une porte, car la grande route venant de Horbourg arrive à cet endroitlà (fig. 27). Les données de la porte sud et la situation du côté oriental restent encore à vérifier.

Les bastions d'angle (fig. 31), où se croisent deux galeries de casernement, constituent une caractéristique architecturale originale. Le seul élément de comparaison est livré par le Palatiolum de Trier-Pfalzel (fig. 32) (Cüppers, 1965), dont un tronçon de mur de $12 \mathrm{~m}$ de hauteur, encore conservé dans l'église collégiale, a permis la restitution des structures d'Oedenburg. Les dimensions intérieures des bastions d'angle, dont chacun est composé de la pièce centrale $(7,60 \mathrm{~m})$ et des deux pièces saillantes $(4 \mathrm{~m})$ sont absolument identiques dans les deux fortifications.

On retrouve dans les techniques de construction de la fortification d'Oedenburg certaines caractéristiques typiques des constructions de l'époque valentinienne dans les régions du Rhin supérieur : pour accroître la stabilité du terrain, une série de piquets est enfoncée au fond des tranchées de fondation à parois verticales, au fond desquelles sont posés un ou plusieurs radiers superposés de poutres entrecroisées (fig. 33). Les caissons formés par les poutres du radier sont remplis de mortier de chaux coulé en alternance avec des lits de pierres.

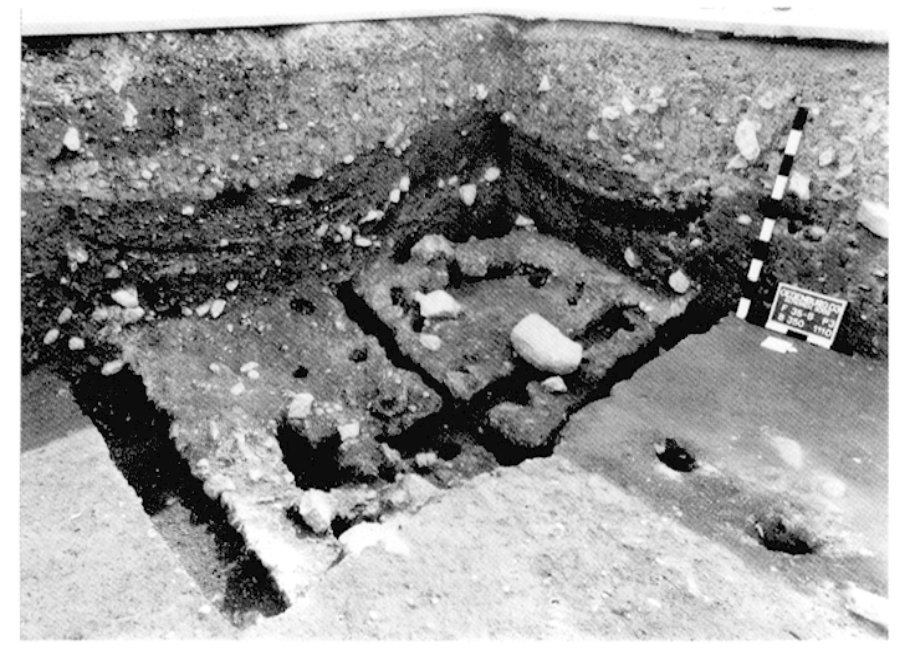

Fig. 33 - Carrés 35/36 d'Oedenburg-Altkirch : vue depuis le nord-est sur les fondations coulées (US 350/1110) avec trous des piquets et négatifs des poutres du radier horizontal (cliché G. Seitz, univ. de Fribourg-en-Brisgau). 


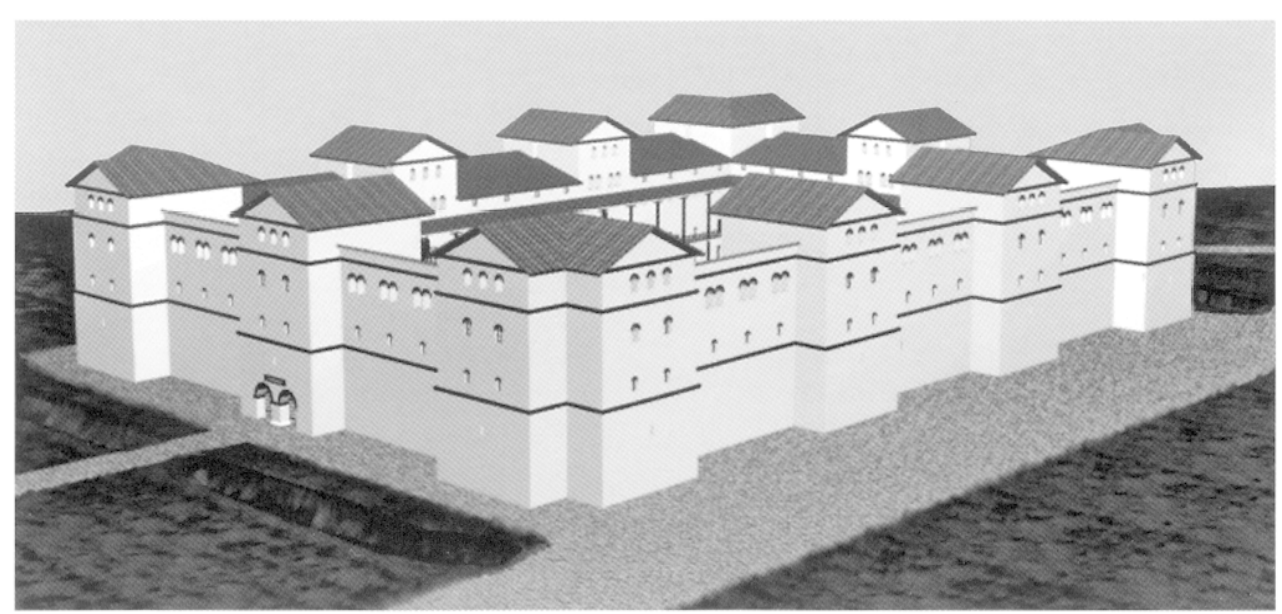

Fig. 34 - Essai de reconstitution de la fortification d'Oedenburg-Altkirch : vue depuis le nord-ouest (dessin S. Feldhusen et S. Berg, univ. de Fribourg-en-Brisgau).
Cette technique a été utilisée à Altkirch. On la retrouve sous le mur intérieur et les murs de refend des casernements; en revanche, au fond des tranchées de fondation presque entièrement spoliées du mur extérieur, seule la présence des piquets a pu être mise en évidence à ce jour. Il n'a pas été possible de déterminer la hauteur exacte des fondations; en ce qui concerne l'élévation des murs, il faut se contenter des exemples connus ailleurs.

\section{Datation, fonction}

La technique de construction caractéristique, mais surtout les nombreuses monnaics, dont le pic date incontestablement de l'époque de Valentinien et de Valens (Biellmann, 1997, p. 18) - comme d'ailleurs les monnaies trouvées sur le fond du fossé - attestent une construction de la fortification sous Valentinien I dans les années 369-374 apr. J.-C. Deux monnaies en argent de Constantin III (407-411) peuvent être considérées comme la preuve d'une présence romaine après les années catastrophiques de 406-407. Chronologiquement, ces deux pièces sont suivies par une autre monnaie d'argent de Théodoric (418-451) (Biellmann, 1997).

La porte sud est reliée à la rocade sud-nord par une route qui se raccorde à celle-ci à une centaine de mètres vers le sud (fig. 5 et 27 ). Une autre route raccorde la porte nord à la rocade à une distance de $250 \mathrm{~m}$. La garnison de la fortification pouvait ainsi surveiller la voie principale sud-nord qui passait directement à côté de la fortification, ainsi que celle venant de l'est qui traversait le Rhin et se dirigeait à l'ouest vers Horbourg.

Mis à part la forme particulière de la fortification (fig. 32 et 34), pour laquelle il n'existe jusqu'à présent aucun autre exemple, excepté à Trier-Pfalıel, les structures archéologiques, en raison de leur mauvais état de conservation, ne nous livrent pas d'indices sur sa fonction précise. Il n'existe aucun niveau de circulation conservé qui puisse nous renseigner au moins sur l'aménagement et la fonction des pièces situées au niveau du sol. Il en va autrement de Trier-Pfalzzel, où non seulement le nom mais également la présence de mosaïques témoignent d'un riche environnement. Cependant, la forme de la fortification d'Oedenburg montre une influence impériale qui pourrait résulter du fait qu'un architecte de la cour a été chargé de sa planification et de sa réalisation. Les armes sont plutôt rares, à l'exception de deux hastae plumbatae (fig. 35, $\mathrm{n}^{\circ} 6$ ) ; le nombre des éléments vestimentaires militaires n'est guère plus élevé : boucles de ceinture, fibules (fig. 36). Soulignons la découverte d'un lingot d'argent du Bas-Empire (Maurer, 1993), d'une bague en or (Biellmann, 2000, p. 21) et d'au moins neuf fibules cruciformes dorées (fig. 36, B 84.99) de type Keller VI (Zagermann, 2003).

\section{L'histoire postromaine}

Les fouilles réalisées dans le secteur de la fortification, également appelé autrefois Kirchenbuckel ou Altkirch, nous ont toujours laissé supposer qu'un jour nous découvririons les vestiges du village disparu d'Oedenburgheim. Mais c'est seulement en 2003 que nous avons pu mettre en évidence dans les carrés 59-62, au sud du cimetière connu, les restes d'au moins trois églises, dont les datations ne sont pas encore établies, et notamment de deux absides (Pétry, 1982a et b). Seule la fin de cette occupation peut être connue : en 1620, il n'y a plus de curé (Schlaefli, 1983), et, à partir de 1696, les ruines de la fortification d'Altkirch ont été systématiquement démantelées.

Les questions relatives à l'histoire du site de l'époque postromaine sont particulièrement intéressantes. La découverte de céramiques peu nombreuses mais significatives (fig. $35, \mathrm{n}^{\circ \mathrm{s}} 3$ et 4) montre qu'après la fin de l'administration romaine au $\mathrm{V}^{\mathrm{e}} \mathrm{s}$., des populations germaniques se sont établies à l'intérieur des murs de l'ancien castrum. À certains endroits au moins, elles ont détruit les murs des casernements romains pour y établir leurs fonds de cabane ou d'autres constructions légères (carrés 60-62). La position des fondations en pierre de l'église montre qu'à l'époque de leur construction, les murs des casernements ne pouvaient plus subsister en élévation. Mais qu'en est-il de la construction d'une première église : a-t-elle été élevée à l'intérieur de bâtiments romains encore existants ou a-t-elle été érigće en bois contre le mur d'enceinte subsistant de la fortification ? 15

H. L. N. et G. S.

15. La traduction du chapitre sur la fortification d'Altkirch a été assurée par Deborah Hacgel, la relecture et la correction de la traduction par Gertrud Kuhnle, et le complément de la traduction du texte par Sibrlle Cournac. 


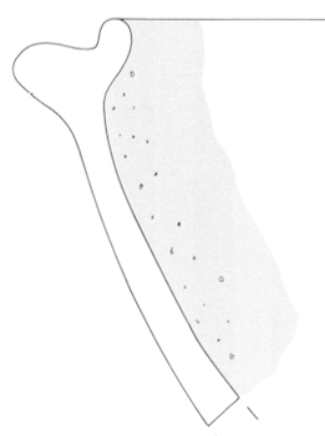

02.03 .1955
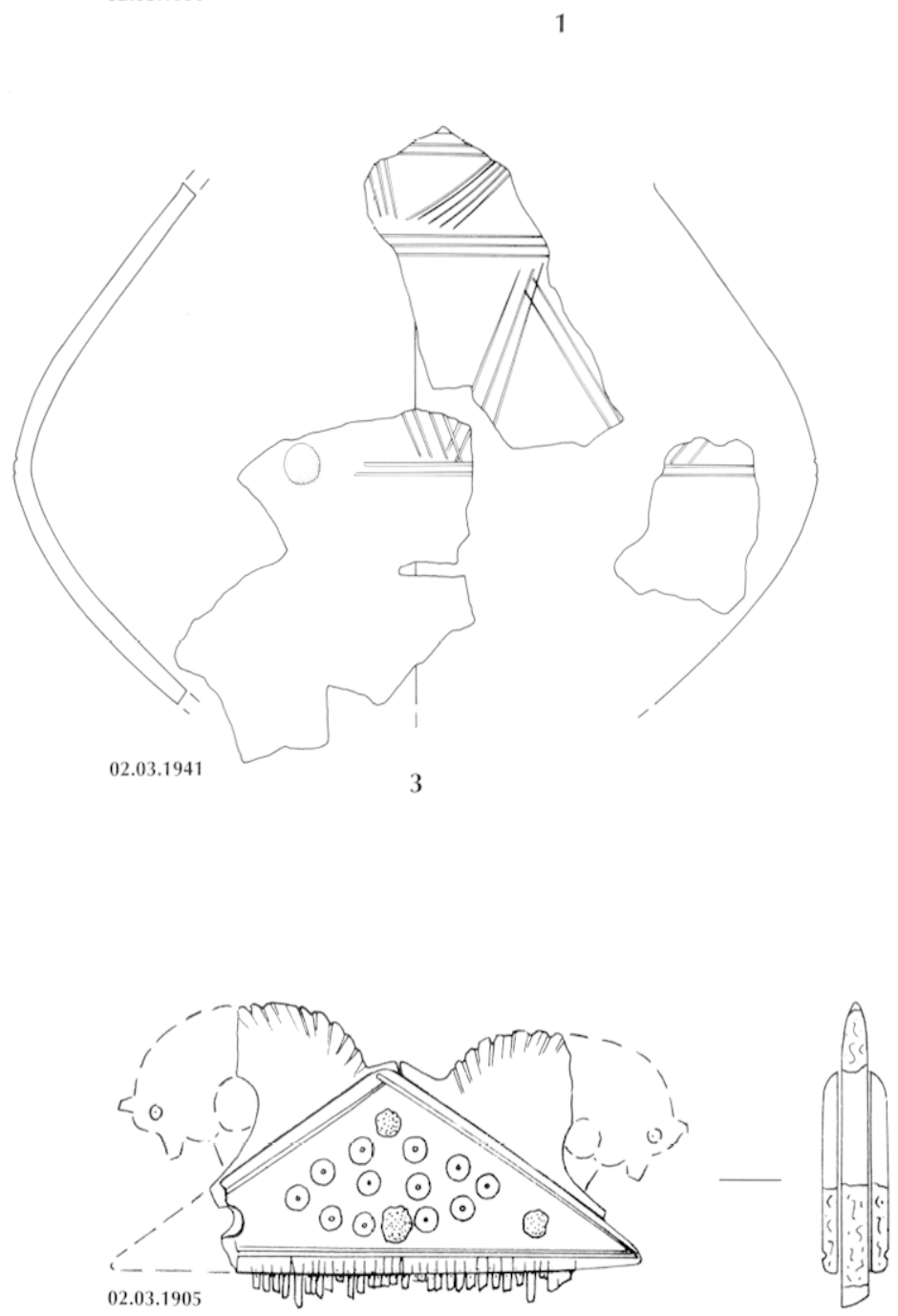

5
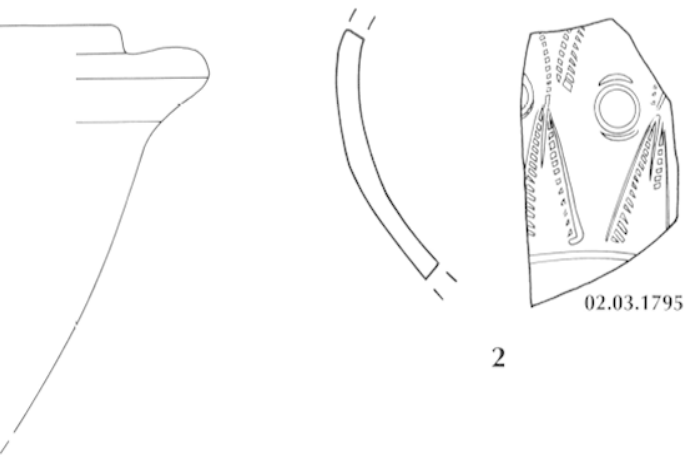

2
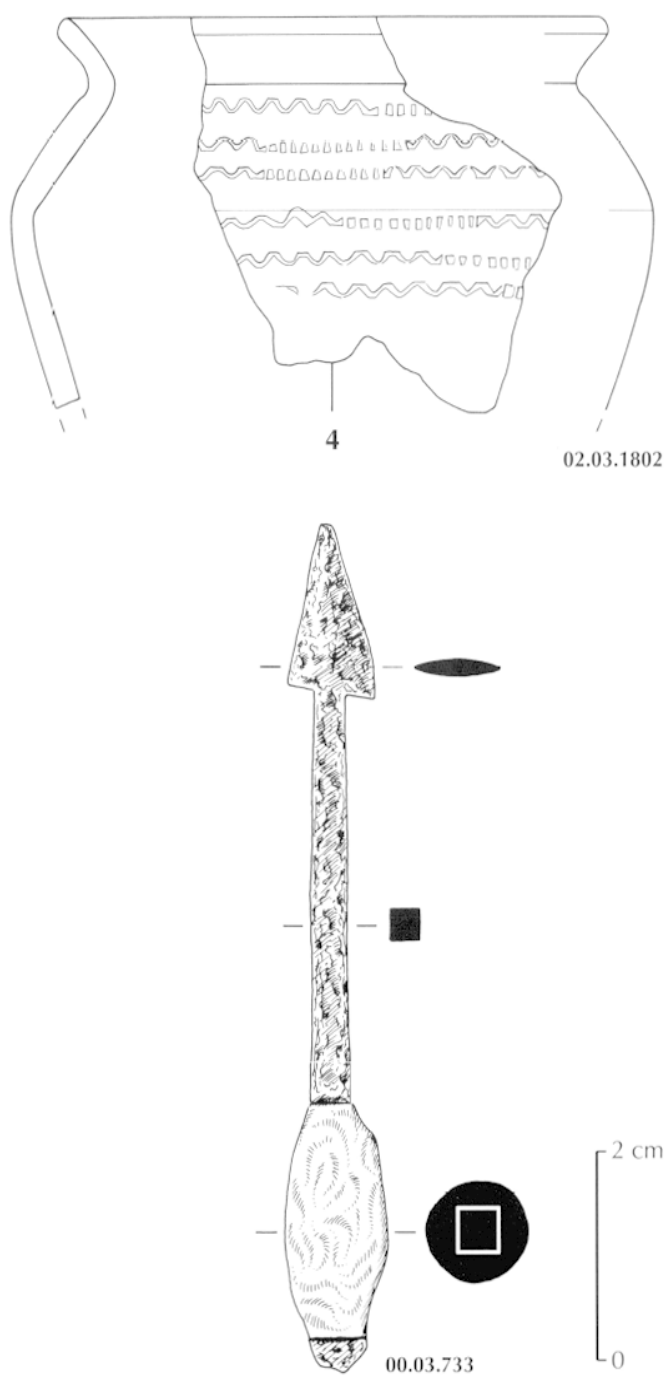

6

Fig. 35 - Céramique tardive, peigne en os et hasta plumbata provenant d'Oedenburg-Alkirch (dessin A. Schwellnus). 


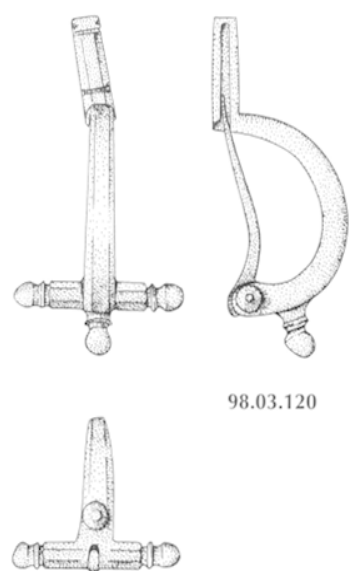

1

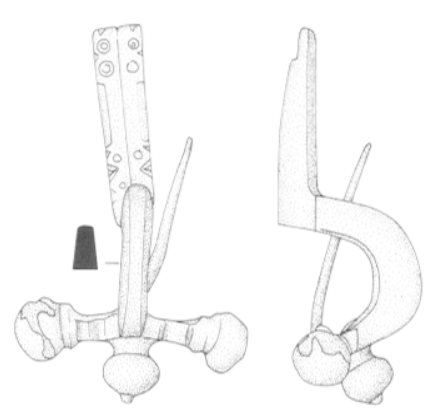

02.03.1694

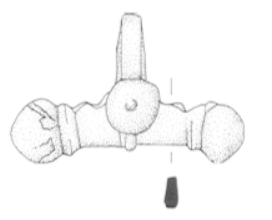

4

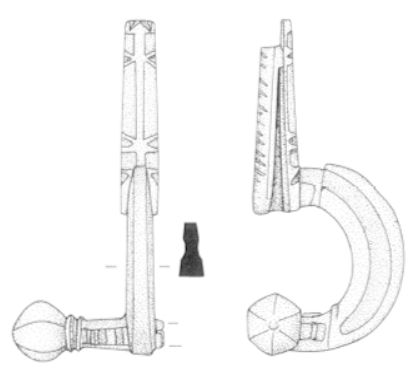

B 99.915

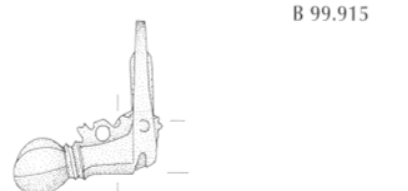

2

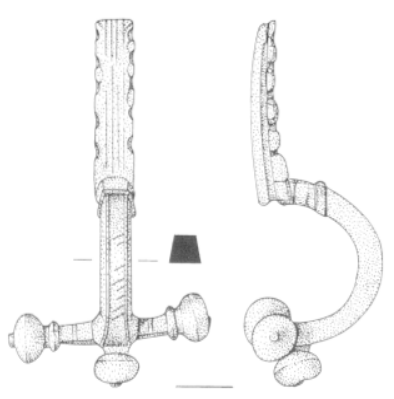

01.03.1274

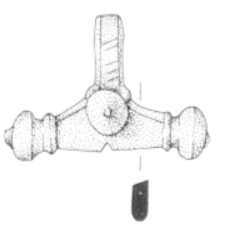

5
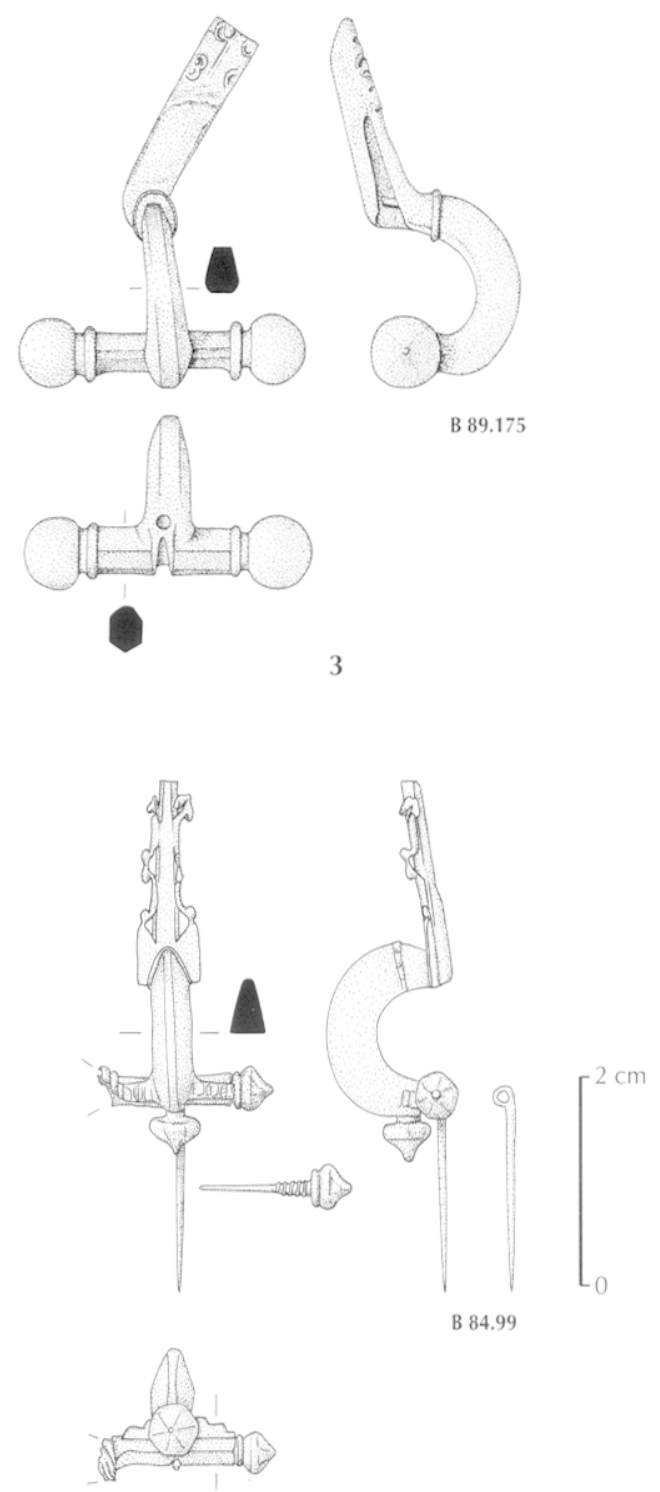

8

Fig. 36 - Différents types de fibules cruciformes provenant d'Oedenburg-Altkirch (dessin A. Schwellnus).

\section{LE MILIEU NATUREL}

\section{ET L'IMPACT DE L'HOMME}

\section{L'AMÉNAGEMENT DU SOL (LECTURE PALÉO- HYDROLOGIQUE DES COUPES DU CHANTIER BK 04)}

Dans le secteur du virus, sur le chantier BK 04, à la sortic occidentale du camp julio-claudien (fig. 8 et 17 ), la réalisation de longues tranchées géoarchéologiques a permis l'observation de la géométrie des dépôts d'un petit paléochenal rectiligne et son interprétation en termes paléohydrologiques. Les relevés géoarchéologiques ont été complétés par des échantillonnages sédimentologiques et micromorphologiques. La chronologie du comblement de ce chenal peut être décrite en six phases successives (fig. 37 et 38 ) :
Phase 1. Lc substratum antérieur à toute occupation antique est constitué de barres de galets et de graviers sableux mis en place dans un système fluviatile en tresse. Ces dépôts sont remaniés et incisés par un chenal rectiligne, subparallèle au canal actuel.

Phase 2. Ce large chenal est comblé par une série argilosableuse homogène jaune, parfois hydromorphe, antérieure aux premières occupations antiques (fig. 37 LS 12, faciès 1). L'analyse granulométrique confirme une mise en place par suspension uniforme (fig. $37 \mathrm{~d}$ ).

Phase 3. Elle se matérialise par la réincision d'un petit chenal orienté nord-sud, d'une vingtaine de mètres de large et d'environ $1 \mathrm{~m}$ de profondeur (berge orientale, fig. 5 et berge occidentale, fig. 38 ). 


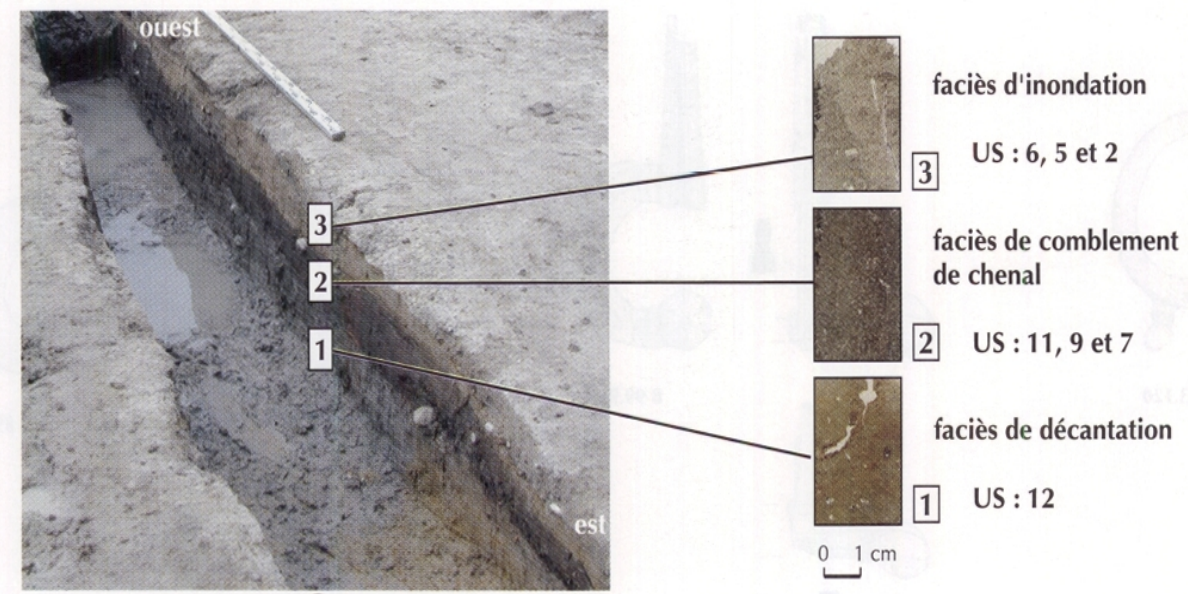

(a)
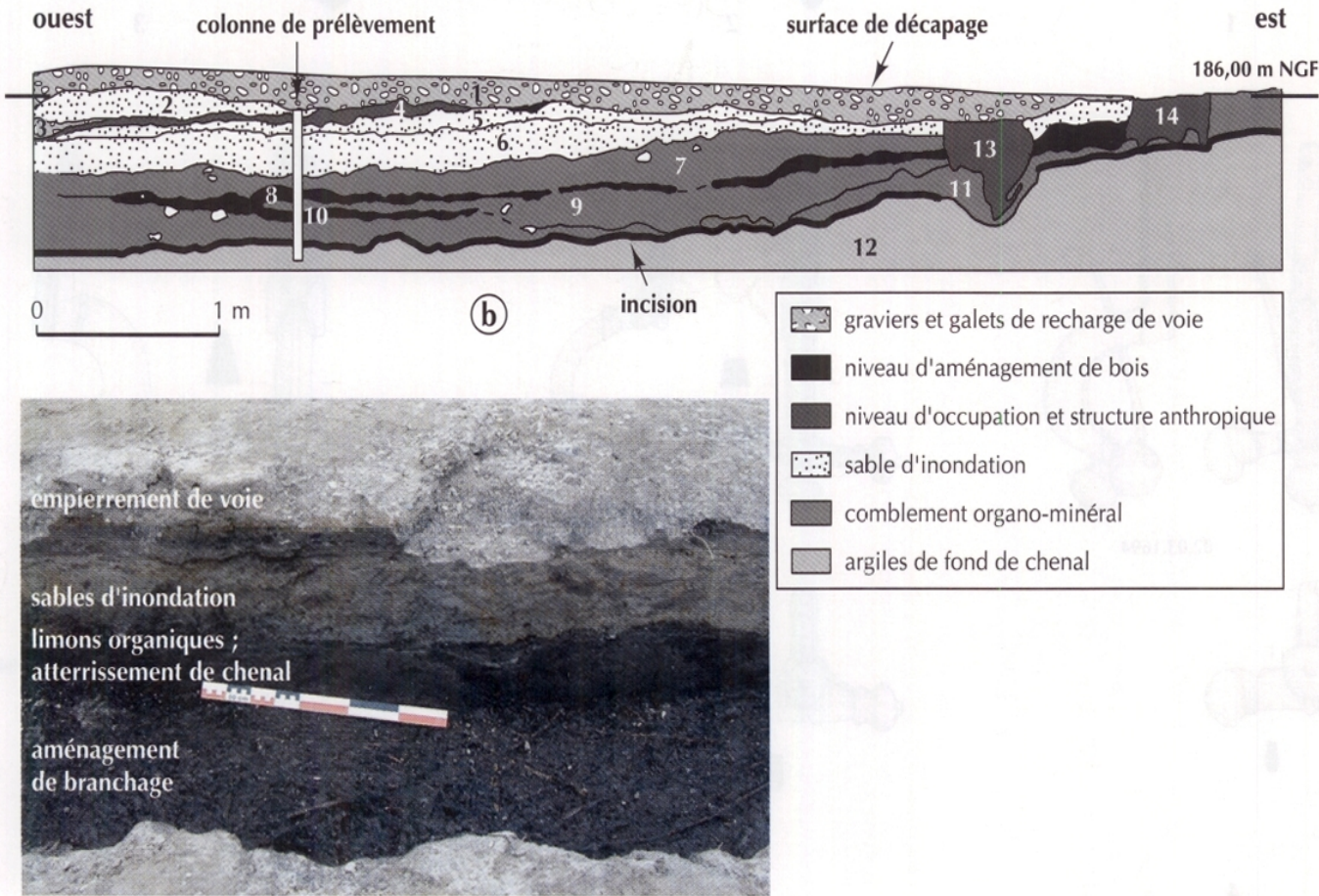

(c)

courbes d'abondance relative

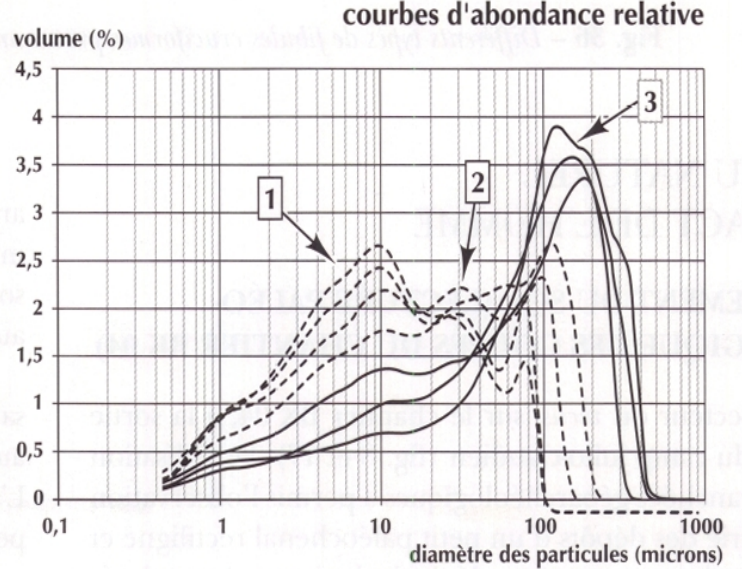

(d)

Fig. 37 - Coupes dans le chantier BK 04 et faciès d'inondation (clichés et dessins C. Petit et V. Ollive, univ. de Dijon). 


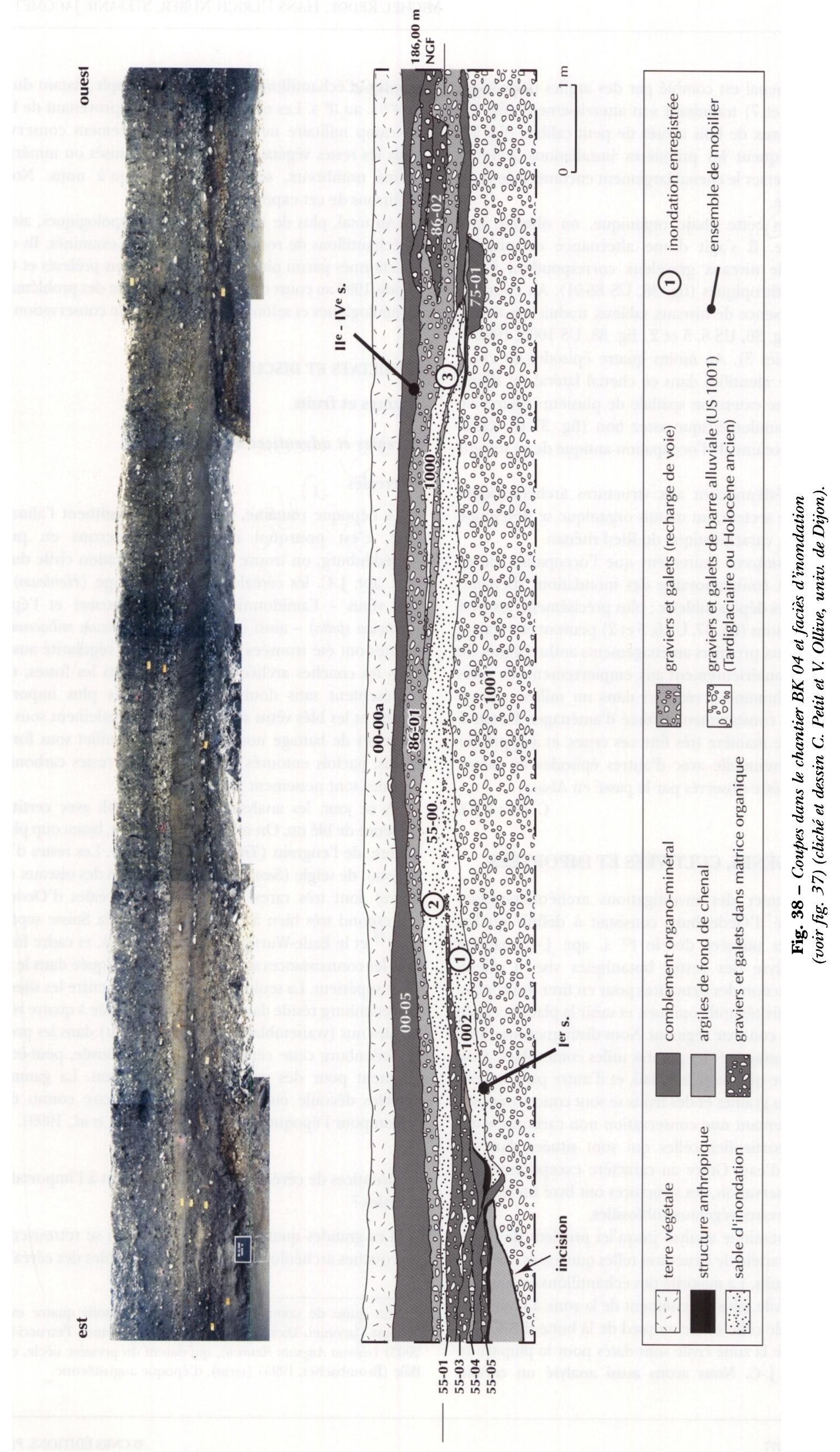


Phase 4. Ce chenal est comblé par des argiles organiques (fig. 37, US 11, 9 et 7) traduisant son atterrissement (fig. 37, faciès 2). Des niveaux de bois tressés de petit calibre (fig. 37, US 10 et 8) marquent les premières installations antiques permettant de traverser le chenal largement encombré de végétation marécageuse.

Phase 5. Après cette phase organique, on observe une période complexc. Il s'agit d'une alternance de niveaux d'inondation et de niveaux graveleux correspondant à des aménagements anthropiques (fig. 38, US 86-01). Au cours de cette phase, la présence de niveaux sableux traduit des événements de crues (fig. 36, US 6, 5 et 2; fig. 38, US 1000, 1002 ct $55-00$; fig. 37 , faciès 3 ). Au moins quatre épisodes de crues peuvent donc être identifiés dans ce chenal latéral du Rhin ; tous présentent une extension spatiale de plusieurs mètres de large et un tri granulométrique assez bon (fig. $37 \mathrm{~d}$ ). Cette phase est contemporaine de l'occupation antique de ce secteur du site.

Phase 6. Postérieurement aux structures archéologiques reconnues dans ce secteur, un marais organique se développe (fig. 38, US 00-05), caractéristique du Ried rhénan.

Ces coupes montrent clairement que l'occupation de ce secteur du site est contemporaine des inondations rhénanes enregistrées par ces dépôts sableux ; plus précisément, les trois premières inondations (fig. 37, US 6,5 et 2) peuvent être datées postérieurement aux premiers aménagements anthropiques du début du I ${ }^{\mathrm{er}} \mathrm{s}$., et antérieurement aux empierrements de voies datés du II ${ }^{*}$ s. L'homme a vécu ici dans un milieu naturel répulsif, qu'il s'est constamment efforcé d'aménager. Il restc à essayer de dater de manière très fine ces crues, et à apprécier leur corrélation éventuelle avec d'autres épisodes de même nature qui ont pu être observés par le passé en Alsace.

$$
\text { C. P. et V. O. }
$$

\section{PLANTES INDIGÈNES, CULTIVÉES ET IMPORTÉES}

L'objectif premier des investigations archéobotaniques menées sur le site d'Oedenburg consistait à définir quelles étaient les plantes utilisées dès le $\mathrm{I}^{\mathrm{er}} \mathrm{s}$. apr. J.-C. par ses occupants. L'analyse des restes botaniques vise à mieux comprendre la fonction des structures pour en tirer des conclusions quant aux activités anthropiques, et saisir la place occupée par le site dans son contexte régional. Nous distinguerons d'une part, les plantes sauvages et les plantes utiles comestibles, aussi bien pour l'homme que pour le bétail, et d'autre part, le bois.

Les analyses des graines et des fruits se sont concentrées sur les structures présentant une conservation non carbonisée des macrorestes, en particulier celles qui sont situées dans les sédiments gorgés d'eau. Grâce au caractère exceptionnel des conditions de conservation, ces structures ont livé un nombre très important de restes végétaux subfossiles.

Le matériel botanique analysé jusqu'ici provient de divers endroits et d'une variété de structures telles que fosses, couches d'occupation ou puits. La majorité des échantillons est issue de l'agglomération civile, plus précisément de la zone à l'ouest du camp militaire et de celle située au pied de la butte d'Altkirch. Les échantillons de la zone civile sont datés pour la plupart du $\mathrm{I}^{\mathrm{er}}$ au III $^{\mathrm{e}}$ s. apr. J.C. Nous avons aussi analysé un certain nombre d'échantillons de la zone du temple, datant du début du $\mathrm{I}^{\mathrm{er}} \mathrm{s}$. au $\mathrm{II}^{\mathrm{e}} \mathrm{s}$. Les matières organiques provenant de la zone du camp militaire ne sont que médiocrement conservées et seuls les restes végétaux fossilisés (carbonisés ou minéralisés), moins nombreux, sont parvenus jusqu'à nous. Nous les exclurons de cet exposé.

Au total, plus de 180 échantillons carpologiques, ainsi que 40 échantillons de restes de bois, ont été examinés. Ils ont été sćlectionnés parmi plus de 700 échantillons prélevés et tamisés depuis 1999 au cours des fouilles, sur la base des problématiques archéologiques et selon leurs conditions de conservation.

\section{RÉSULTATS ET DISCUSSION}

\section{Graines et fruits}

\section{Céréales et adventices de céréales}

\section{- Céréales}

À l'époque romaine, les céréales constituent l'aliment de base, c'est pourquoi nous les évoquerons en priorité. À Oedenburg, on trouve dans l'agglomération civile du $\mathrm{I}^{\mathrm{cr}}$ au $\mathrm{II}^{\circ}$ s. apr. J-C. les céréales suivantes : l'orge (Hordeum), deux blés vêtus - l'amidonnier (Triticum dicoccum) et l'épeautre (Triticum spelta) - ainsi que le millet (Panicum miliaceum). Ces plantes ont été trouvées avec une grande régularité aussi bien dans les couches archéologiques que dans les fosses, et clles représentent sans doute les céréales les plus importantes. L'orge et les blés vêtus se trouvent principalement sous forme de restes de battage non carbonisés; le millet sous forme de grains, parfois entourés des glumes. Les restes carbonisés de céréales sont nettement plus rares.

À ce jour, les analyses n'ont pas établi avec certitude la présence de blé nu. On relèvera la présence, beaucoup plus irrégulière, de l'engrain (Triticum monococcum). Les restes d'avoine (Avena), de seigle (Secale cereale) et de millet des oiseaux (Setaria italica) sont très rares. L'éventail des céréales d'Oedenburg correspond très bien à celui connu pour la Suisse septentrionale ${ }^{16}$ et le Bade-Wurtemberg (Stika, 1996), et cadre fort bien avec les connaissances sur l'agriculture pratiquée dans le sud du Rhin supérieur. La seule différence notoire entre les sites bâlois ct Oedenburg réside dans la présence, attestée à quatre reprises, de blés nus (vraisemblablement du froment) dans les premiers. À Oedenburg cette céréale n'a pas été trouvée, peut-être uniquement pour des raisons de conservation. La gamme des céréales découle en droite ligne du spectre connu dans la région pour l'époque de La Tène (Jacomet ot al., 1999).

- Adventices de céréales - des indices quant à l'importation de céréales?

Les grandes quantités d'adventices qui se retrouvent dans les couches archéologiques sont indissociables des céréales (ou

16. En guise de comparaison, nous avons choisi quatre exemples d'Augst (Jacomet, Dick, 1986 ; Dick, 1989 ; Jacomet, Petrucci-Baraud, 2003) (colonia Augusla Raurica), qui datent du premier siècle, et un de Bâle (Brombacher, 1995) (vicus), d'époque augustéenne. 
de leurs résidus de battages). Grâce aux excellentes conditions de conservation présentes dans la zone de l'agglomération civile d'Oedenburg, 34 taxons ont pu être dénombrés. La gamme des adventices typiques des céréales d'hiver retiendra plus particulièrement notre attention. En effet, nous avons repéré quelques espèces qui n'apparaissent que très rarement, voire jamais, dans des niveaux romains du nord des Alpes.

En premier lieu, on trouve partout la nielle des blés (Agrostemma githago), le myagre perfolié (Myagrum perfoliatum), le chénopode blanc (Chenopodium album) et le mouron des oiseaux (Stellaria media). La nielle des blés semble connaître une diffusion particulièrement large ; en effet, elle est présente en importantes quantités dans certaines fosses, en particulier là où l'on relève aussi la présence de matières fécales. Voilà qui correspond à ce que l'on peut par exemple observer dans les latrines médiévales (Jacomet et al., 1993). Les graines de nielle des blés étant toxiques, leur consommation en grandes quantités peut devenir problématique (Knörzer, 1970; Pals, Hakbijl, 1992).

Pour la région du Rhin supérieur (et aussi pour toute la Suisse et pour le Bade-Wurtemberg ${ }^{17}$ ), les taxons suivants sont nouveaux ou très rares à l'époque romaine : Ajuga chamaepitys, Myagrum perfoliatum, Orlaya grandiflora, Caucalis platycarpos, Ranunculus arvensis, Scandix pecten-veneris (détermination à confirmer) et Vaccaria pyramidata. Il faut encore ajouter le bleuet (Centaurea cyanus) à cette liste. Certains de ces taxons sont caractéristiques des cultures de céréales d'hiver sur sols calcaires (alliance du Caucalidion).

Il est très difficile de déterminer si l'ensemble de ces taxons poussait dans la région d'Oedenburg au début du $\mathrm{I}^{\mathrm{er}}$ s. apr. J.-C. Théoriquement, ils pouvaient croître à proximité immédiate, non loin du Rhin où des cailloutis riches en calcaire sont présents juste sous la couche d'humus. Avant la mise en place des cultures industrielles de maïs et l'utilisation d'herbicides, la majorité des mauvaises herbes évoquées étaient présentes dans la région. La plupart des auteurs (par ex. Wiethold, 1998) considèrent des espèces telles que Orlaya grandiflora comme des introductions romaines. Sur la base des connaissances actuelles, la présence de ces mauvaises herbes " exogènes ", au $\mathrm{I}^{\mathrm{er}} \mathrm{s}$. apr. J.-C. pourrait indiquer l'importation de céréales du sud de la France, bien que l'on ne trouve pas de céréales "exotiques".

Dans ce contexte, on relèvera plus particulièrement la présence très régulière de pointes de cosses de Myagrum perfoliatum. À Oedenburg, elles comptent parmi les trouvailles d'adventices les plus fréquentes, alors qu'à ce jour de tels vestiges n'ont été découverts que très rarement dans des sites romains du nord des Alpes. La présence de Myagrum vient corroborer l'hypothèse de l'importation de céréales. Myagrum est une adventice annuelle d'hiver (Hegi, 1958, IV). Aujourd'hui, on ne la trouve que très rarement dans les champs, et généralement de manière peu constante (surtout parmi les céréales et le colza), mais aussi dans les zones en friche, les prairies, les lisières, les terres en friche (gravières, gares), le long des rivières, etc. Sa répartition se concentre surtout dans le sud de l'Europe.

17. Par exemple, dans le Bade-Wurtemberg, on ne les rencontre pas à l'époque romaine (Stika, 1996).
On rencontre encore à Oedenburg toutes sortes d'adventices présentes aussi sur d'autres sites, comme les chénopodes, Fallopia convolvulus, Galium aparine (Tap), Agrostemma githago et bien d'autres. Leur répartition naturelle est très large, si bien que leur provenance ne peut être définie.

\section{Autres plantes utiles - l'importance des plantes importées}

Les niveaux humides du site ont livré de très nombreuses plantes utilisées par l'homme, en particulier des plantes comestibles. Dans les sites romains de la Suisse nord-occidentale examinés à ce jour (Augst et Bâle), la plupart de ces plantes ne sont presque jamais présentes. On relèvera qu'il s'agit uniquement de trouvailles carbonisées, puisque ces deux sites se trouvent dans des sols minéraux bien drainés. Les restes de fruits, de noix, de légumes, etc. proviennent surtout de fosses.

\section{- Noix et noisettes}

Noisettes (Corylus avellana) et noix (Juglans regia) ont été régulièrement trouvées. Ce sont les Romains qui ont introduit la noix dans nos régions. Si on a trouvé des noisettes dans la plupart des fosses, la répartition des noix est moins régulière.

En Suisse, les noix sont très rares au $\mathrm{I}^{\mathrm{er}}$ s. apr. J.-C., surtout au cours des cinquante premières années. La plus grande quantité découverte à l'époque augustéenne dans toute l'Europe centrale provient d'un entrepôt de commerçant ou lieu de stockage à Windisch (Vindonissa) antérieur au camp militaire; il s'agit vraisemblablement de noix importées (Jacomet, 2003). La seule autre découverte de noix d'époque augustéenne provient du vicus de Bâle. Au début de l'occupation romaine, les noix semblent toujours être en relation avec un contexte militaire (Jacomet et al., 2002 ; Jacomet, 2003). Plus tard, elles deviennent plus fréquentes, sans doute en relation avec la mise en place de cultures locales. En Suisse, il semblerait que ce phénomène apparaisse au cours de la seconde moitié du $\mathrm{I}^{\mathrm{er}}$ s. apr. J.-C., mais c'est plus une hypothèse fondée sur l'image globale fournie par les macrorestes qu'un fait prouvé (Jacomet, Petrucci-Bavaud, à paraître). Dans l'est de la France, la présence de la noix est attestée par des pollens d'une fosse en milieu humide, découverte à Besançon et datant du $\mathrm{I}^{\mathrm{er}} \mathrm{s}$. apr. J.-C (Passard, Urlacher et al., 1997). En 2003, quelques structures d'Oedenburg ont été soumises à l'analyse palynologique. Celles-ci ont révélé la présence de pollens de noix dans une seule structure (03-05-16) de la zone du temple. Ceci peut indiquer la présence de noyers à Oedenburg, mais seulement à proximité de l'aire sacrée. Ainsi, les noix restaient probablement des produits importés.

\section{- Plantes oléagineuses et fibreuses}

Parmi les plantes oléagineuses et fibreuses, le lin (Linum usitatissimum), le pavot (Papaver somniferum), l'olive (Olea europaea) et le chanvre (Cannabis sativa) ont été repérés ${ }^{18}$. Il est

18. Bien entendu, la majorité de ces taxons peut être utilisée à d'autres fins que l'extraction d'huile ou de fibres : le pavot est une plante médicinale ou une drogue ; le lin peut aussi être ajouté aux plats, et les olives peuvent servir d'assaisonnement. 
probable que l'on connaissait aussi la caméline cultivée (Camelina sativa). Dès que l'on est en présence de milieux gorgés d'eau, le lin et le pavot sont régulièrement présents sur les sites romains, alors qu'ils sont plus ou moins inconnus en milieu terrestre. Ainsi, ces taxons sont attestés en Suisse dans les couches gorgées d'eau du I ${ }^{\mathrm{er}}$ s. apr. J.-C. des vici de Soleure (Salodurum), d'Oberwinterthur (Viludurum) et d'Eschenz (Tasgaetium) (Jacquat, 1986 ; Jacomet et al., 1993 ; Feigenwinter, 1997; Pollmann, 2003). Ces considérations sont également valables pour le Bade-Wurtemberg (Stika, 1996). La présence de ces deux plantes n'est donc pas exceptionnelle en soi. En revanche, la découverte de chanvre et d'olives reste relativement rare. On ne les rencontre par exemple jamais dans le BadeWurtemberg (Stika, 1996). En Suisse, on connaît pour le $\mathrm{I}^{\mathrm{er}} \mathrm{s}$. apr. J.-C. quelques fragments d'olives provenant de Windisch et antérieurs au camp légionnaire (Jacomet, 2003), plusieurs découvcrtes en contexte funéraire réalisées à W'indisch (Petrucci-Bavaud, Jacomet, 2000) et à Arconciel (PetrucciBaraud, non-publié), ainsi que diverses trouvailles anciennes à Avenches (Aventicum) (Baas, 1987 et 1990). Il est certain que les olives étaient importées des côtes de la Méditerranée.

\section{- Légumes et salades, en particulier la calebasse}

Les restes d'une série de légumes et de salades ont été trouvés à Oedenburg, parmi lesquels on compte aussi des légumineuses. Ce groupe de plantes est surtout bien représenté en contexte humide, à l'exception des légumineuses.

L'amarante (Amoranthus sp.) et l'arroche (Atriplex sp.) apparaissent avec régularité. Les graines de chou (Brassica sp.) ont été repérées asse\% fréquemment. Parmi les légumineuses, on compte la lentille (Lens culinaris), le pois (Pisum sativum) et la fève (Vicia faba). Ces trois légumineuses sont fréquemment présentes dans les niveaux archéologiques romains. Le statut de la lentille n'est pas encore clair : en Suisse, on la rencontre souvent dans de riches sépultures ${ }^{19}$, signe qu'elle était consommée par la frange aisée de la population. On relèvera encore la présence de la carotte (Daucus carota), de la chicorée/endive (Cichorium sp.) et du pourpier (Portulaca oleracea). La détermination de ce dernicr reste à confirmer.

Toutes les espèces mentionnées jusqu’ici ont en commun d'avoir été, partiellement il est vrai, introduites par les Romains ${ }^{20}$, mais elles peuvent avoir été cultivées dans ces contrées. Voilà qui ne va pas de soi pour la calcbasse (Lagenaria siceraria). Celle-ci est originaire de l'Afrique tropicale et demande un climat plus chaud. Dans la structure 99-04-01, nous avons trouvé non seulement les graines, mais aussi le pédoncule et un fragment du fruit d'une calebasse (Nuber, Reddé et al., 2002). Dans la structure 03-09-74, une calebasse presque entière avec son pédoncule a été repérée (fig. 39). Cette découverte est exceptionnclle, ćtant donné que nous ne

19. Voir par exemple la tombe à bustum du propriétaire de la villa de Biberist-Spitalhof, (Jacomet, à paraître), ou les très riches tombes à incinération de Reinach BI. (Mausackerweg) (Schlumbaum, PetrucciBavaud, s.d.).

20. Toutefois, certaines d’entre elles ćtaient déjà présentes en contexte laténicen (Kreuz, 2002).

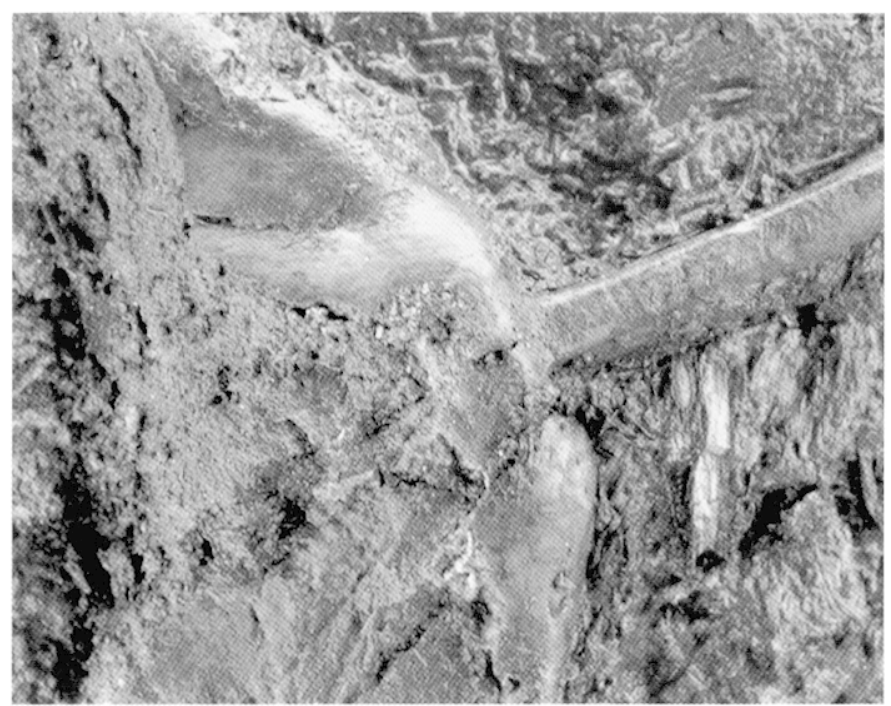

Fig. 39 - Fragment du fruit de calebasse (Lagenaria siceraria) provenant de la structure 03-09-74 (cliché P. Vandorpe, univ. de Bâle).

connaissons à ce jour, pour l'époque romaine, qu'un seul vestige semblable trouvé à Longueuil-Sainte-Marie. Il s`agit d'un fragment d'environ un tiers du fruit, représentant l'extrémité distale (Marinval et al., 2002). De manière générale, la découverte du fruit de calebasses est très rare; nous avons connaissance de trouvailles d'autres époques à Houten (PaysBas), datant du haut Moyen Âge (Kooistra, Hessing, 1988) et à Oberflacht datant du XIV s. (Schiek, 1992).

Pour les sites romains, les restes de calebasses font aujourd'hui cncore figure d'exception : on ne connaît que peu de sites qui en aient livré, et la plupart ne datent que du $\mathrm{II}^{\circ} \mathrm{s}$. apr. J.-C. Les restes découverts à Oedenburg, dans la zone du temple, datant de la première moitié du I ${ }^{\mathrm{er}}$ s. de notre ère, sont les plus anciens. En Allemagne, on ne connaît la calebasse qu'à Dieburg (Göldner, Kreuz, 1999) (vicus), Ellingen (Frank, Stika, 1988) (camp), Hanau-Kesselstadt (Kreuz, 1994) (vicus se rattachant à un camp) et Lahr-Dinglingen (Rösch, 1995) (vicus). En Suisse, scul un puits du vicus de Petinesca a livré des calebasses ${ }^{21}$. À Oedenburg, la calebasse apparaît assez fréquemment, et il s'agit de la plus importante quantité connue à l'époque romaine pour les provinces situées au nord de l'Arc alpin.

Un essai réalisé au cours de l'été 2000 au jardin botanique de Bâle nous a montré que les calcbasses, en tant que légumes, peuvent être cultivées sous nos latitudes (Jacomet, ErnyRodmann, 2000). Il est cependant peu vraisemblable d'obtenir des calebasses lignifiées: le climat est en effet trop humide. Pour pouvoir confirmer la culture des calebasses à Oedenburg, il faudrait découvrir des structures telles que celles de Longueuil-Sainte-Marie (Oise) : là, un jardin compartimenté a été trouvé en parallèle de la découverte de calebasses (De Hingh, 1993). Le contexte de découverte des vestiges de calcbasses à Oedenburg ne permet pas une appréciation de l'importance de cette plante, puisque sa conservation n'est de toute évidence possible qu'en milieu gorgé d'eau.

21. Laboratoire de Bâle, en cours d'élaboration. 
- Plantes aromatiques : poivre, anis, etc.

À Oedenburg, les plantes aromatiques sont également bien représentées. Presque tous les taxons sont parvenus dans nos régions grâce aux Romains. Les espèces les plus abondantes sont celles que l'on trouve fréquemment, en particulier en milieu humide ou en présence de matériel minéralisé. Il s'agit de l'aneth (Anethum graveolens), du coriandre (Coriandrum sativum), les "épices-standards " des Romains, et du céleri (Apium graveolens) ${ }^{22}$. Les découvertes d'anis (Pimpinella anisum) et de poivre (Piper nigrum) méritent une attention toute particulière. Des macrorestes de ces deux épices ont été trouvés dans la structure 00-04-24, qui date du $\mathrm{I}^{\mathrm{er}}$ s. apr. J.-C., et un autre grain de poivre (qu'il conviendra de déterminer avec davantage de précision) dans la structure 02-04-15 ( $\mathrm{II}^{\mathrm{e}} \mathrm{s}$. apr. J.-C.). La découverte d'un fruit d'anis devra faire l'objet d'une vérification. En effet, il s'agirait de la première découverte de l'époque romaine au nord des Alpes. L'analyse palynologique a relevé un pourcentage très haut de pollens d'anis dans la structure 03-09-74. Le grain de poivre découvert en 2000 a été, quant à lui, identifié avec certitude. À l'époque romaine, le poivre est un bien de luxe, importé d'Inde ; même les sources antiques le décrivent comme une denrée inutile et le qualifient de très coûteux (par exemple Pline, Histoire naturelle, XII, 29). À ce jour, les preuves directes, sous forme de fruits, de la présence de poivre sont très rares. Hors Oedenburg, on n'a découvert des grains de poivre que dans les latrines d'un centurion dans le camp légionnaire d'Oberaden (époque augustéenne) (Kucan, 1992), dans un puits du vicus rattaché au camp de Hanau-Kesselstadt (II' s. apr.J.-C.) (Kreuz, 1994) et dans la zone portuaire de Straubing en Bavière ( $\mathrm{I}^{\mathrm{er}} \mathrm{s}$. apr. J.-C.) (Küster, 1995). Il faut encore mentionner la découverte d'une étiquette en plomb appartenant à un sac de poivre et découverte à Trèves (Schwinden, 1985). La mise au jour de poivre à Oedenburg correspond sans doute à une première en France pour l'époque romaine.

\section{- Fruits}

À Oedenburg le groupe des plantes à fruits est très richement représenté, grâce à la présence de sédiments gorgés d'eau. Il peut être divisé en deux sous-groupes :

- les espèces d'origine indigène, récoltées dans leur milieu naturel, comme la fraise des bois, le coqueret alkékenge, la prunelle, l'aubépine, la framboise, la mûre et le sureau ;

- les espèces introduites dans nos régions par les Romains, comme le melon ou le concombre, la figue, la mûre noire, la cerise, la prune, le pruneau, la pêche et le raisin. Le statut des poires et des pommes est peu clair, les trouvailles ne permettant pas de différencier la forme sauvage de la forme cultivée.

Les espèces du deuxième sous-groupe, en particulier, n'ont pratiquement jamais été attestées à Augst et à Bâle au $\mathrm{I}^{\mathrm{er}} \mathrm{s}$.

22. À l'époque romaine, le céleri-rave, la forme que nous conmaissons le mieux, n'existait pas encore. D'après les sources écrites (André, 1998), on consommait les branches comme légumes, ou les petits fruits pour assaisonner les plats - ces derniers ont été retrouvés à plusieurs reprises. apr. J.-C. ${ }^{23}$, alors qu'elles étaient présentes dans nos régions à cette époque comme le montrent les grandes quantités de la plupart de ces taxons, attestés par exemple à Soleure, Oberwinterthur et Eschenz dans des sédiments gorgés d'eau (Jacquat, 1986; Jacomet et al., 1993; Feigenwinter, 1997; Pollmann, 2003). Dans le Bade-Wurtemberg, on dispose également de nombreuses découvertes (Stika, 1996). Il n'est donc guère étonnant d'en rencontrer de grandes quantités à Oedenburg. Les fruits les plus fréquemment observés sont les figues, les pommes ou poires, les cerises, les prunes, ainsi que les pêches, les prunelles, les mûres, les framboises, les raisins et les sureaux. Ces plantes étaient souvent consommées.

On rencontre aussi à Oedenburg des plantes qui sont très rares en d'autres endroits, surtout au $\mathrm{I}^{\mathrm{er}} \mathrm{s}$. apr. J.-C. On citera entre autres le melon ou le concombre (Cucumus melo/satious) et le mûrier noir (Morus nigra). En Allemagne, on dénombre à ce jour cinq sites ayant livré des melons ou concombres : le vicus de Dieburg, datant du II ${ }^{\mathrm{e}}$ au III' s. apr. J.-C. (Göldner, Kreuz, 1999), le camp d'Ellingen, occupé au II ${ }^{\mathrm{e}}$ s. apr. J.-C. (Frank, Stika, 1988), le vicus de Baden-Baden (Gernsbacher Strasse) datant de la fin du ${ }^{\text {er }}$ s. apr. J.-C. (Stika, 1996), la villa d'Eschborn-Dörnweg (du $\mathrm{II}^{\mathrm{e}}$ au $\mathrm{III}^{\mathrm{c}}$ s. apr. J.-C.), ainsi que le vicus de Nidderau-Heldenbergen ( $\mathrm{II}^{\mathrm{e}} \mathrm{s}$. apr. J.-C.) (Baas, 1982). Les restes de mûrier noir n'ont pas encore fait l'objet de recherches systématiques. On connaît des découvertes faites dans les puits de certaines villue du Rheinland (Knörzer, Meurers-Balke, 1990). En Suisse, ces taxons ne sont attestés que depuis fort peu de temps grâce aux travaux menés à Bâle (Pollmann, 2003 ; Klee, Jacomet, 2003).

On relèvera la découverte de raisin (Vitis vinifera). Outre des milliers de pépins, nous avons aussi trouvé des fragments du fruit. Les points noirs observés à leur surface montrent, selon une communication orale de $\mathrm{J}$. Wiethold, qu'il pourrait s'agir de raisins secs. Certains pépins sont mal développés, caractère que l'on retrouve dans la vigne cultivée. À notre connaissance, il s'agit de trouvailles uniques.

\section{Autres groupes de végétaux}

Outre les groupes végétaux évoqués, de nombreux autres taxons sont apparus. Il s'agit, pour une part, de plantes poussant dans les décombres, que l'on rencontre volontiers dans les déblais, les arrière-cours, les bords de chemin, etc., plus généralement les endroits riches en azote. Bon nombre d'entre elles se développent très bien comme mauvaises herbes. Ces plantes, en particulier la renouée à feuilles de patience/ persicaire (Polygonum lapathifolium/persicaria), la renoncule rampante (Ranunculus repens) et la patience sauvage (Rumex obtusifolius) se trouvent avec une grande régularité aussi bien dans les fosses que dans les niveaux archéologiques. Ces espèces faisaient sans aucun doute partie de la végétation locale des zones d'habitat, comme c'est le cas de plantes riveraines (poussant en des endroits humides et riches en phosphates), à l'exemple de Polygonum hydropiper ou de Polygonum mile/minus. Ces taxons nous indiquent que l'emplacement était humide et,

23. Il s’agit généralement de fragments carbonisés dont la détcrmination est incertaine. 
selon nos critères modernes, plutôt souillé. Ces considérations sont surtout valables pour la zone de l'ancien bras du Rhin, dont proviennent les niveaux riches en bois. D'autre part la découverte sporadique de plantes aquatiques indique que l'on y trouvait aussi de l'eau stagnante. On imagine une mare riche en phosphate. La zone civile, à l'ouest du camp militaire, devait, au I ${ }^{\text {er }}$ s. apr. J.-C., être peu " accueillante ", et il n'est guère étonnant que l'on ait cherché à l'assécher : couches de branches-brindilles et copeaux de bois, suivies de remplissages de gravier (voir supra, p. 231 et 250-252).

Nous avons aussi identifié des plantes poussant en milieu ouvert, dont la provenance est difficile à déterminer avec précision. Une grande quantité de ces espèces n'a pas encore fait l'objet d'une détermination détaillée, comme par exemple les nombreuses herbacées, lamiacées, brassicacées, etc.

\section{Bois}

Au total, plus de 1300 bois provenant de structures gorgées d'eau de l'agglomération civile ont été déterminés ${ }^{24}$. Il s'agit, d'une part, de bois non travaillés, d'autre part, d'artefacts bien reconnaissables.

Dans l'agglomération civile, plusieurs couches de branches et de brindilles ont été trouvées (entre autres dans les structures 01-04-50, 02-0455, 02-0478). Il semble que l'on ait tenté, dès le début de l'occupation romaine, d'assécher cette zone située dans un paléochenal. Ces branches proviennent surtout d'essences " locales ", voire d'arbres poussant à proximité immédiate des rives du Rhin, comme le saule (Salix sp.), l'aulne (Alnus sp.), particulièrement indiqué pour la construction sur sol humide, et le peuplier (Populus $s p$.). On ne trouve que très rarement d'autres espèces: le noisetier (Corylus avellana), la viorne (Viburnum $s p$.), le pin (Pinus $s p$.). En outre, les déchets $\mathrm{du}$ travail du bois et autres déchets de bois ont été répandus. Ici, le spectre d'espèces est plus riche. Parmi les bois utilisés, on compte l'aulne, le sapin blanc (Abies alba), l'épicéa (Picea abies), l'érable champêtre (Acer campestre), l'érable sycomore/érable plane (Acer pseudoplatanus/Acer platanoides), le noisetier, le bouleau (Betula sp.), le chêne (Quercus $s p$.) et le hêtre (Fagus sylvaticus).

Plusieurs artefacts ont été repérés dans l'agglomération civile. Trois objets méritent une mention particulière: un fragment de planchette à brosses, un fragment de tablette à écrire et un peigne. Le fragment de planchette à brosses est en bois de hêtre; les soies, dont la partie se trouvant dans les perforations a été conservée, n'ont pas encore été examinées. Les découvertes de brosses romaines sont rares. On en connaît par exemple dans les fouilles anciennes du dépotoir de Windisch ${ }^{25}$, du vicus d'Oberwinterthur (Fellmann, 1991) et dans le vicus d'Eschenz (Brem et al., 1999). Il semble que les planchettes composant la base des brosses n'aient pas été taillées dans un bois unique. La production de planchettes en

24. A. Schlumbaum a effectué la détermination de l'ensemble des bois. 25. Site fréquemment cité mais non publié ; la liste du mobilier est déposée auprès du service d'archéologie cantonale d'Argovie. La détermination des bois a été faite par W. Schoch, la détermination des soies par A. Heitz-Weniger et l'ensemble a été catalogué par R. Fellmann (Fellmann, 1991). hêtre, telles que celle qui a été rencontrée à Oedenburg, est à ce jour inconnue ailleurs.

Le fragment de planchette à écrire comporte une bordure en relief munie d'une petite perforation. Il s'agit d'épicéa. Les tablettes à écrire romaines étaient presque toujours en résineux, soit en épicéa, épicéa/mélèze (Picea abies/Larix decidua) ou en sapin blanc (Fellmann, 1991).

C'est sans doute une découverte remarquable que celle d'un petit peigne en buis (Buxus sempervirens) comportant deux rangées de dents et long de $6 \mathrm{~cm}$ environ. On peut le comparer à des trouvailles en buis réalisées à Eschenz et à Oberwinterthur (Hedinger, Leuzinger, 2002).

Comme nous l'avons dit, le saule, le peuplier et l'aulne sont des représentants caractéristiques de la forêt alluviale pionnière bordant les rives du Rhin. À une distance plus importante du fleuve, on trouvait la forêt alluviale au stade terminal comprenant des espèces indigènes très appréciées, comme par exemple le chêne, surtout le chêne pédonculé (Quercus robur), l'orme (Ulmus $s p$.) et le frêne (Fraxinus sp.). S'y trouvaient certainement aussi le noisetier, la viorne et l'érable champêtre.

Sur les hauteurs de la basse terrasse, la forêt devait correspondre à celle que l'on rencontre aujourd'hui dans le milieu sec de Colmar : forêts de chênes pubescents avec quelques pins, des érables champêtres, quelques charmes (Carpinus $s p$.), des érables planes et des noisetiers. Ces forêts étaient certainement exploitées, du moins pour y ramasser du bois de chauffage.

Si l'on se base sur les conditions actuelles, le sapin blanc, le hêtre, et probablement aussi l'érable sycomore, l'épicéa et le buis ne poussaient pas à proximité immédiate du site. Ces bois provenaient de zones éloignées. Les forêts les plus proches se trouvent dans les Vosges, à quelques kilomètres d'Oedenburg, à une altitude de plus de $500 \mathrm{~m}$. Le sapin blanc compte parmi les espèces de bois les plus fréquentes sur le site. Il semble que les Romains aient exploité les populations de sapins blancs des Vosges de manière intensive. Le buis, tout comme l'érable sycomore, provient vraisemblablement aussi de cette région.

Il est difficile de déterminer la provenance de l'épicéa. Les crêtes de la Forêt-Noire correspondent aux emplacements naturels les plus proches, et l'épicéa pousse aussi en des endroits spécifiques dans le Jura ${ }^{26}$. C'est dans les Alpes que l'on rencontre des populations d'épicéa plus importantes. Sa présence à Oedenburg est particulière, du fait que deux tablettes à écrire étaient confectionnées en bois de cette espèce. Il faut sans doute ici favoriser l'hypothèse de l'importation des tablettes à écrire en épicéa.

Globalement, l'éventail des espèces ligneuses découvertes montre d'une part que les bois poussant à proximité immédiate ont fait l'objet d'une exploitation intensive, d'autre part, que l'on avait régulièrement recours à des objets importés, en particulier des Vosges.

\section{Interprétation des structures selon l'ensemble des plantes répertoriées}

Un des buts de l'analyse des restes végétaux est de vérifier si les restes botaniques permettent d'éclairer la fonction de struc-

26. Il semble que la présence de l'épicéa dans les Vosges ne soit pas naturelle (communication orale de $\mathrm{H}$. Zoller). 
tures particulières. Les sédiments étudiés se rapportent à la dernière fonction des structures et ne permettent pas d'aborder en détail la question de leur utilisation primaire ou secondaire.

Quelques fosses particulièrement "riches" ont livré des indices clairs de la présence de matière fécale : au moment de leur remplissage, elles servaient aussi (ou peut-être surtout) de latrines. Nous considérons la présence de concrétions minéralisées ${ }^{27}$ comme un indice de déjections, ainsi que des pourcentages élevés de son de céréales non carbonisées (on notera que ces derniers n'ont à ce jour pas été comptés systématiquement); il en va de même des fragments de graines de nielle des blés, d'amas de cellules cristallisées de poires et de petites graines de plantes aromatiques ou de fruits comme le céleri et la figue. En outre, les pupes de mouches sont également des indicateurs de matière fécale. Pour le $\mathrm{I}^{\mathrm{er}} \mathrm{s}$. apr. J.-C., les fosses 00-04-24 et 00-04-27 dans la zone à l'ouest du camp étaient riches en éléments de ce type. Pour le $\mathrm{II}^{\mathrm{c}}$ s. apr. J.-C., on peut avancer avec certitude que la fosse 00-04-38 a été utilisée comme latrine (et dépotoir). D'autres fosses riches en résidus botaniques n'ont livré que peu ou pas d'indicateurs de matière fécale, comme par exemple la fosse 02-04-15 du II ${ }^{\mathrm{e}} \mathrm{s}$. apr. J.-C. Il y avait donc des fosses utilisées en premier lieu comme latrines, d'autres qui servaient uniquement de dépotoirs et d'autres encore utilisées peut-être à des fins particulières.

\section{L'importance d'Oedenburg dans la zone du Rhin supérieur d'après les découvertes botaniques}

Oedenburg a livré un nombre très important de taxons végétaux "exotiques ». Il s'agit du melon ou du concombre, de la calebasse, de l'olive, vraisemblablement de l'anis, du poivre ainsi que du mûrier noir. Des dattes carbonisées ont été trouvées dans un vase d'offrande dans l'agglomération civile lors des fouilles de 2000. L'analyse des pollens de la structure 01-04-24 a établi la présence de pistache. À notre connaissance, il ne s'agit que de la deuxième découverte de pistache au nord des Alpes ${ }^{28}$. Comparé à la liste des taxons végétaux " exotiques " rares et d'ordinaire importés établie par Bakels et Jacomet (2002), on note à Oedenburg l'absence d'amande, de riz, de pignon de pin, de grenade et d'ail. Pratiquement toutes les autres plantes " exotiques " importées connues en Europe centrale ont été découvertes. On est dès lors tenté d'attribuer au site une importance majeure, puisque des personnages en mesure de s'offrir des biens d'importation fort côtteux $y$ auraient séjourné. Dans un premier temps, il s'agissait peut-être d'officiers du camp militaire, plus tard également d'autres personnages.

Toutefois, il est permis de se demander si cette conclusion correspond bien à la réalité : si l'on disposait pour l'époque romaine de plus de niveaux archéologiques conservés à l'état humide et bénéficiant d'analyses aussi systématiques que celles pratiquées à Oedenburg, ces taxons perdraient sans doute une bonne part de leur caractère exceptionnel.

27. À ce sujet, voir Jacomet, 2003 (Windisch-Breite).

28. Ln reste de pistache a été retrouvé à Windisch dans des niveaux augustéens (Jacomet, 2003).
Une alternative pour interpréter la richesse des restes végétaux " exotiques " à Oedenburg pourrait être sa localisation. Le site se trouve non loin du bord du Rhin, celui-ci étant à l'époque romaine une voie de communication très importante entre le sud et le nord de l'Empire romain. Les habitants avaient ainsi accès à ces biens luxueux et le site pourrait avoir été un lieu de redistribution des marchandises destinées au ravitaillement des villae plus éloignées du fleuve.

S. J., A. S., P. V. et L. W.

\section{LA FAUNE}

\section{LES DONNÉES}

\section{Importance des échantillons de faune des différentes campagnes de fouille}

Les restes osseux recueillis et étudiés dans le cadre des différentes campagnes de fouille organisées entre 1999 et 2002 forment un ensemble de 12543 restes, auxquels s'ajoutent 7514 autres fragments issus des refus de tamis de maille supérieure à $4 \mathrm{~mm}$, ainsi que 484 pièces provenant des tamis à maille de 1 à $4 \mathrm{~mm}$. Au total ce sont donc 20541 restes qui ont été prélevés, représentant une masse de $156,1 \mathrm{~kg}$ (sans les restes de tamisage). Si l'on considère l'importance des échantillons réunis au cours des différentes campagnes, il apparaît que, l'année 2000 mise à part, le nombre de restes prélevés n'a cessé d'augmenter de campagne en campagne. Ainsi pour environ 5000 ossements analysés en 1999, ce sont presque 6000 restes qui ont été pris en compte en 2001, tandis qu'en 2002 ce nombre s'élève à presque 7800 pièces. Sur la base de ces données, on peut développer des comparaisons chronologiques et régionales, tant entre les différentes structures du site qu'entre Oedenburg et les autres contextes régionaux.

\section{Les différents ensembles}

Les structures ${ }^{29}$ étudiées ont été classées en différents ensembles sur la base de critères chronologiques et spatiaux. Celles du camp du $\mathrm{I}^{\mathrm{er}}$ s. (environ 25-70 apr. J.-C.) sont regroupées sous le terme de "camp ". Les découvertes situées immédiatement à l'extérieur de la porte ouest sont réunies dans un deuxième ensemble appelé "camp/vicus », dans la mesure où leur caractérisation, militaire ou civile, n'est pas assurée pour l'instant. S'y rattachent aussi les autres structures de la même 7one pour lesquelles une datation analogue à celle du camp peut être proposée (environ 25-70 apr. J.-C.). Toutes les structures datées principalement du dernier quart du $I^{\text {er }}$ s., soit celles du chantier 04, ainsi que celles du chantier 05 ( ${ }^{\mathrm{er}} \mathrm{s}$. apr. J.-C.) sont réunies sous le terme de "vicus du $\mathrm{I}^{\text {cr }} \mathrm{s}$. apr. J.-C. ". Le quatrième ensemble, "vicus du $\mathrm{II}^{\mathrm{e}}$ s. apr. J.-C. ", rassemble toutes les structures civiles des chantiers 04 et 05 datées du II ${ }^{\mathrm{e}}$ s. apr. J.-C. Les restes osseux issus du sondage “BK 02-08 " dans le Riedgraben (fig. 8) sont, dans l'attente d'une étude détaillée de ce chantier, décomptés à part, dans un cinquième ensemble, et ne sont pas pour l'instant intégrés aux données des autres chantiers. Enfin tous les complexes gallo-

29. Ensembles anatomiques non intégrés. 
romains tardifs sont regroupés au sein d'un ensemble "Westergass tardif " ${ }^{30}$. Les données se répartissent ainsi entre six entités aux dénominations suivantes ${ }^{31}$ :

. " camp " : NR $=974 ; 6831,7$ g; NR tamisé $=2079$;

. "camp/vicus" : NR = $3723 ; 36484,4 \mathrm{~g} ; \mathrm{NR}$ tamisé $=1527$;

. "vicus $\mathrm{I}^{\mathrm{er}}$ s. apr. J.-C. " : NR $=1119 ; 12602,1 \mathrm{~g}$; NR tamisé

$=2489$;

"vicus $\mathrm{II}^{\mathrm{C}}$ s. apr. J.-C. " : NR = $2212 ; 17419,2$ g ; NR tamisé

$=1663$.

"BK 02-08": NR = $1102 ; 18$ 892,1 g; NR tamisé : 0 ;

. «Westergass tardif » : NR $=1920 ; 37068,0 \mathrm{~g} ; \mathrm{NR}$ tamisé $=0$.

\section{Les données collectées à vue}

L'ensemble le plus important est celui du "camp/vicus" qui englobe six structures parmi lesquelles la fosse-dépotoir 99-04-01 (fig. $17 \mathrm{f}$ et voir supra, p. 231) concentre à elle seule $80 \%$ des vestiges. La composition des autres ensembles est marquée par une répartition moins déséquilibrée. Celle du " camp " intègre quatre complexes aux caractéristiques très homogènes, constitués de comblement de fosses appartenant à la dernière occupation du " grand camp " dont les échantillons de faune, d'importance à peu près égale, comptent entre 150 et 400 pièces.

Représentant environ $10 \%$ des restes osseux, l'ensemble de la faune du "camp " est d'importance égale à celui du " vicus du I ${ }^{\text {er }}$ s. " et du sondage "BK 02-08 ". Les vestiges datés des II $^{\mathrm{e}}$ et $\mathrm{IV}^{\mathrm{e}} \mathrm{s}$. forment respectivement $20 \%$ et $17 \%$ des données de base. Globalement, plus de la moitié des données se rapportent à des ensembles de restes datés du I ${ }^{\mathrm{cr}} \mathrm{s}$. apr. J.-C.

\section{CARACTÉRISTIQUES DES VESTIGES OSSEUX COLLECTÉS À VUE (1999-2002)}

\section{Poids moyen des restes et taux de détermination}

Le taux de détermination, d'environ $92 \%$ sur la base du poids des restes, est relativement élevé, et ne varie que très peu entre les différents ensembles. Sur la base du nombre de restes, la part des fragments déterminés varie entre 35 et $80 \%$. Le faible taux de détermination qui caractérise l'ensemble de la faune de "Westergass tardif "s'explique par la prédominance d'ossements très fragmentés, dont le poids moyen ne dépasse pas $3 \mathrm{~g}$. Le reste de la faune issue de cette \%one est en revanche constitué de gros éléments, relativement lourds et dont la détermination ne pose pas de problèmes particuliers. Les poids moyens relativement élevés qui caractérisent les vestiges de l'ensemble «BK 02-08 " se rapportent probablement au fait qu'il s'agit de restes issus de sondages. Mais le taux de détermination assez bas, qui, dans cet ensemble, ne dépasse pas $50 \%$, pourrait par ailleurs aussi résulter de conditions de conservation défavorables. Dans le cas des quatre autres cnsembles, le poids moyen des restes est d'environ $9,1 \mathrm{~g}$, variant entre $12,9 \mathrm{~g}$

30. Une sélection d'ensembles de faune du chantice BK 99-02, datés de la période gallo-romaine tardive, a fait l'objet d'un mémoire de diplôme (Ginclla, 2002 [inédit]).

31. Le terme vicus désigne ici, de manière conventionnelle, l'agglomération rivile d'Oedenburg, sans préjuger de son statut juridique réel, toujours inconnu. dans le cas des restes déterminés et $1,4 \mathrm{~g}$ pour les indéterminés : valeurs comparables à celles d'autres ensembles fouillés récemment (Pfäffli, Schibler, 2003). À titre de comparaison, les ossements issus des fouilles anciennes à Augst présentent un poids moyen nettement plus élevé, variant entre 20 et $65 \mathrm{~g}$ (Schibler, Furger, 1988, fig. 4).

Le taux de détermination particulièrement élevé, associé au faible poids moyen qui caractérise les ossements du " camp ", s'explique, d'une part, par la prédominance de restes d'espèces de faible gabarit et, d'autre part, par leur conservation dans des structures de faible volume, à l'abri de toute source de fragmentation secondaire.

\section{Liste et importance relative des espèces en présence}

La plupart des espèces domestiques attestées à l'époque romaine sont présentes sur le site : "équidés ", bœuf, mouton, chèvre, porc et chien, ainsi que, parmi la volaille, la poule et, quoique plus rarement, l'oie et le pigeon. Sous l'appellation " équidés "sont regroupés les ossements susceptibles de se rapporter au cheval mais aussi à l'âne ou au mulet. La distinction ostéologique de ces espèces, sur la base d'ossements ou de restes dentaires, est délicate, sinon impossible, du fait de l'absence de clés de distinction précises. Les déterminations menées jusqu'à présent permettent d'attester de façon sûre la présence du cheval et du mulet. D'autres indices relatifs à l'existene du mulet à Oedenburg proviennent aussi d'ensembles non pris en compte dans cette étude ${ }^{32}$.

Aucun reste n'a pu être attribué de façon sûre à l'âne. Ia question de la composition exacte de la population d'équidés reste cependant encore largement à préciser par une étude ostéologique plus poussée.

\section{La faune sauvage}

Des restes isolés d'espèces sauvages sont attestés dans presque toutes les structures. Parmi les gibiers figurent le cerf (Cervus elaphus), le chevreuil (Capreolus capreolus), le chamois (Rupicapra rupicapra), le sanglier (Sus scrofa) et le lièvre (Lepus europaeus). L'occurrence régulière de restes de lièvres atteste la présence de paysages ouverts dans l'environnement proche du site. La question de l'origine du chamois reste ouverte, car plusieurs possibilités peuvent être envisagées : la Forêt-Noire, les Vosges ou encore le Jura. Les données de recherches biologiques concernant des populations modernes et celles d'études archéozoologiques de sites suisses ont permis d'établir que le chamois était originellement une espèce de milieux escarpés et plutôt forestière (Baumann et al., 2005).

Globalement, le rôle économique des espèces sauvages paraît insignifiant sur ce site (fig. 40). Leurs vestiges ne représentent qu’à peine $2 \%$ du nombre de restes. De façon signifi-

32. Des restes signalant la présence du mulet ont été reconnus dans différents ensembles de faune, comme ceux du camp militaire de Dangstetten sur la rive droite du Rhin dans le Bade-Wurtemberg (Uerpmann, 1977) et du relais romain de Salodurum sur le plateau suisse. Des indices sûrs n'existent pas non plus dans les ensembles de faune récemment étudiés de la cité d'Augusta Raurica (par ex. Grädel, 1989 ; Furger, Deschler-Erb, 1992). En contexte militaire, l'importance du mulet s'explique par son utilisation comme animal de bât et de transport (Deschler-Erb, 1999). 
cative, la majeure partie de ces restes cst associée au "camp ", ce qui laisse supposer que la chasse était réservée aux classes supérieures du personnel militaire (Ginella el al., 2000 ; Pfäffli, Schibler, 2003). Il sera particulièrement intéressant de préciser cet aspect, par la prise en compte, dans les études à venir, du mobilier ostéologique issu des casernements des officiers et de leurs latrines. Dans le chantier « BK 02-08 », l'absence d'ossements d'animaux sauvages n'est vraisemblablement qu'un effet de l'état d'avancement des travaux, car la plupart des restes d'oiseaux qui composent ces ensembles n'ont pas encore été déterminés jusqu'au rang de l'espèce. Les résultats des déterminations de l'avifaune, des poissons et de la microfaune disponibles sont résumés infra, p. 261.

\section{Le bæuf domestique (Bos taurus)}

Prédominant dans tous les ensembles, sauf dans le " camp ", le boeuf domestique est l'espèce dont les restes sont les plus fréquents (fig. 40). Ceux-ci représentent entre 40 et $65 \%$ du nombre de fragments. Sur la base du poids des restes, cet animal constitue même la ressource carnée la plus importante, contribuant pour plus de la moitié à l'approvisionnement en viande. Comme la vocation première de l'élevage bovin était de fournir des animaux pour le transport des denrées ou l'exploitation agricole (attelage, bât), l'abattage et l'exploitation bouchère ne représentent qu'une valorisation ultime d'animaux de réforme. La consommation de cette viande de moindre qualité est probablement plutôt le fait de milieux sociaux peu aisés, à supposer que ceux-ci aient pu s'offrir de la viande. Une consommation plus importante de veaux (environ $40 \%$ ) n'est attestée qu'au sein des ensembles issus du "camp ", tandis que, dans toutes les autres structures, ces animaux ne représentent qu'entre 20 et $30 \%$ des restes, ce qui souligne d'autant mieux le caractère discriminatoire de la consommation de cette viande. Si l'on admet que la plus grande partie de la population du vicus du $\mathrm{II}^{\circ}$ s. est d'origine civile, il faut se rendre à l'évidence que, d'après l'importance de la représentation du bœuf, elle ne bénéficie pas d'un statut social très élevé. Il reste à déterminer si les taux, également très élevés et de datation proche $\left(\mathrm{II}^{\mathrm{e}} \mathrm{s}\right.$.), des restes de bovins dans le chantier "BK 02-08 ", traduisent la même réalité. Comme l'étude de rejets culinaires apporte avant tout des renseignements qui concernent le rôle alimentaire des animaux, la faible représentation des bovins dans le " camp " n'est pas forcément significative de leur faible implication dans la vie militaire d'Oedenburg (fig. 40). Elle se rapporte plus précisément au fait que cet animal ne figure pas parmi les ressources alimentaires les plus régulières. Il est vraisemblable aussi que la consommation du boeuf ne faisait pas partie de la culture des militaires de rang supérieur, dont le mode de vic était plutôt influencé par les traditions méditerranéennes. Il est possible aussi que, du fait de sa piètre qualité, la viande de bœuf ne constituait pas un mets prisé par ces militaires, ou du moins pour les plus gradés d'entre eux.

Fig. 40 - Importance relative des principales espèces dans les différents ensembles (pourcentages NR), échelles diverses. linsembles anatomiques non intégrés (DAO F. (Ginella, unir. de Bâle).
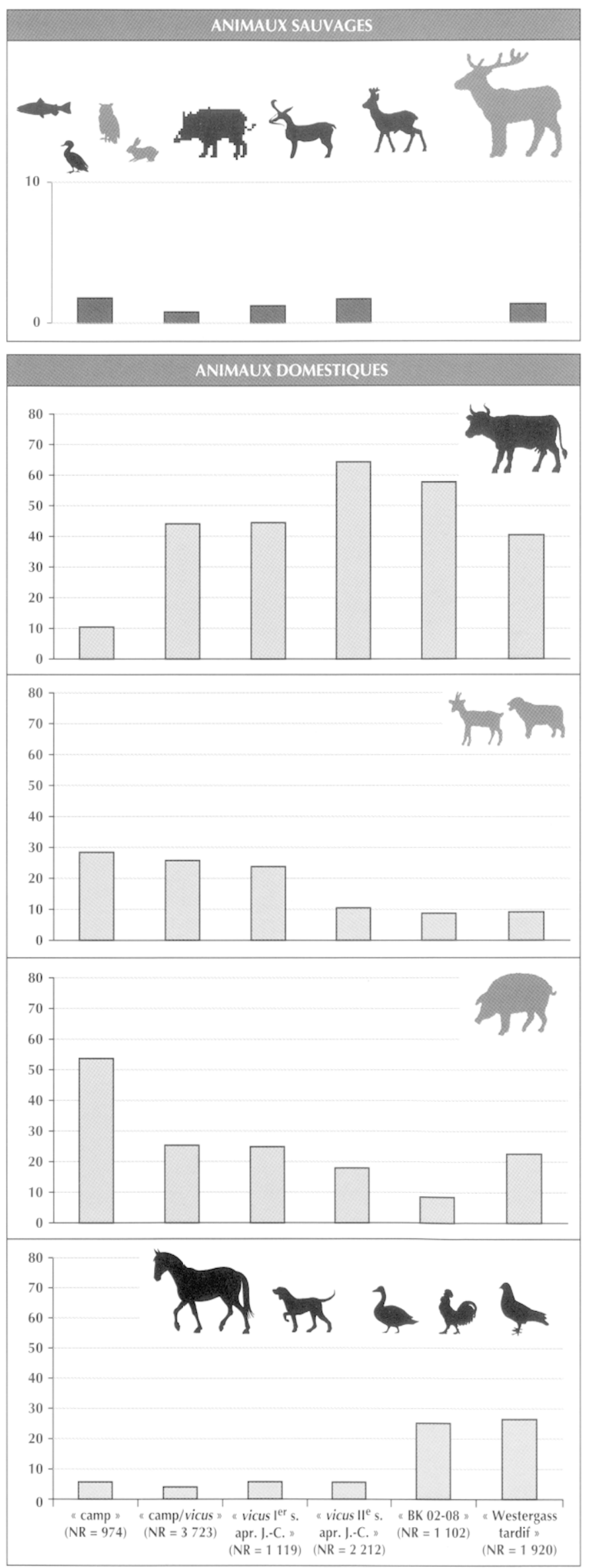


\section{Le mouton et la chèvre (Ovis aries et Capra hircus)}

Dans tous les ensembles datés du $\mathrm{I}^{\mathrm{er}}$ s., les proportions de restes de mouton et de chèvre avoisinent $25 \%$ des échantillons, soit une représentation qui peut être considérée comme relativement importante. Avec plus de $28 \%$ de restes, l'ensemble issu du " camp " s'avère être le plus riche en vestiges de petits ruminants (fig. 40). À partir du $\mathrm{II}^{\mathrm{e}} \mathrm{s}$., la représentation de ces animaux se réduit de moitié pour atteindre des taux qui ne dépassent pas $10 \%$. Une évolution comparable marque les ensembles du site d'Augusta Raurica (Schibler, Furger, 1988) ainsi que d'autres sites de Suisse et du sud de l'Allemagne. Il est possible que cette modification procède d'une influence plus marquée du régime méditerranéen au cours du $\mathrm{I}^{\mathrm{er}} \mathrm{s}$. (surtout la première moitié du $\mathrm{I}^{\mathrm{er}} \mathrm{s}$. apr. J.C.), qui correspond aussi à la période de la plus forte présence de populations, militaires mais aussi civiles, d'origine méridionale. Dans cette région, les petits ruminants occupaient traditionnellement une place plus importante qu'au nord des Alpes. Parmi les sites suisses, ce sont les camps militaires qui, toutes périodes confondues, fournissent les faunes les plus riches en vestiges de mouton et de chèvre (Ebersbach, Schröder, 1997, fig. 4 ; Schibler, à paraître ; Deschler-Erb et al., 2002, fig. 166). La plupart de ces sites sont datés du I ${ }^{\text {er }}$ s. apr. J.C.

Les faibles fréquences de ces petits animaux sont une caractéristique commune aux ensembles datés du $\mathrm{II}^{\mathrm{c}} \mathrm{s}$. et au chantier "BK 02-08 " (fig. 40), ce qui suggère que ce dernier pourrait dater de la même période. Les proportions les plus élevées d'animaux jeunes se concentrent dans les ensembles associés au « camp ", dont elles contribuent à souligner l'origine sociale supérieure.

\section{Le porc (Sus domesticus)}

Dans les ensembles du " camp ", on enregistre une représentation du porc deux fois plus importante que sur le reste du site (fig. 40). Représenté par plus de la moitié des vestiges, cet animal constitue la principale ressource de l'alimentation carnée des militaires. Avec moins de $10 \%$ des restes, la faune du chantier "BK 02-08 " essentiellement constituée de fragments de grands mammifères comme les bovins ou les équidés, s'avère être l'ensemble le moins riche en porc. Dans les autres ensembles datés du $\mathrm{I}^{\mathrm{cr}} \mathrm{s}$. comme dans les complexes tardifs, le porc est représenté par environ 1/4 des ossements déterminés, pour à peine $20 \%$ dans les ensembles datés du II $^{\mathrm{e}}$ s. Principalement vouée à la boucherie, à l'exclusion de toute autre forme de valorisation importante, l'exploitation du porc peut être considérée comme une ressource de choix. Cette orientation s'appuie sur un abattage ciblé des animaux qui atteignent leur maturité pondérale. La rareté des restes se rapportant à des animaux de plus de 2-3 ans découle de cette sélection, soulignée aussi par l'abondance d'ossements non soudés, d'animaux jeunes qui représentent entre 40 et $60 \%$ des restes. La valeur de la viande de porc est par exemple soulignéc par l'édit de Dioclétien (301 apr. J.-C.) qui fixe pour la viande de porc un prix nettement plus élevé que pour celle des autres animaux. La position sociale plus importante que les bouchers porcins occupaient à Rome s'explique par les mêmes raisons. Ces données laissent percevoir une denrée coûteuse dont la consommation régulière était réservée à des cercles de privi- légiés. Le fait que ce soit justement cette ressource qui est la mieux représentée dans le "camp " indique que les déchets de consommation issus de ce secteur correspondent à ceux d'officiers ou d'autres personnels militaires de rang supérieur. Cette représentation importante des restes de porc caractérise également les structures du " camp " fouillées lors de la campagne 2003, autour de la porte nord-ouest. Dans le secteur du "vicus", les restes de porc constituent environ $25 \%$ de la faune, ce qui correspond à un taux de représentation caractéristique d'un milieu social peu élevé, mais équivalent à celui attesté dans d'autres vici en Suisse (Schibler, à paraître ; Deschler-Erb et al., 2002, fig. 166).

\section{Les autres espèces}

La liste des restes des autres espèces reconnues sur le site, ainsi que leur taux de représentation, sont résumés dans la figure 40. Dans cette catégorie sont réunis les restes d'équidés, les ossements de chien et les parties de carcasses de volaille domestique. Les assemblages les plus remarquables caractérisent les ensembles de "BK 02-08 " et de "Westergass tardif ". Ces deux lots de faune se singularisent par des taux élevés de restes d'équidés (Nuber, Reddé et al., 2002) provenant de la gestion de cadavres (dans le cas de "Westergass") et dont le statut n'a pas grand chose à voir avec celui de déchets culinaires. La présence en proportions plus importantes de restes d'équidés dans le chantier « BK 02-08 " peut s'expliquer par une concentration intentionnelle des restes osseux les plus encombrants de bovins, aussi bien que d'équidés. Le chien n'est attesté qu'exceptionnellement par des restes isolés. La structure 22, repérée au cours de la campagne 2002 dans le chantier 05 , constitue un cas particulier, puisqu'elle a principalement servi à l'ensevelissement de cadavres de chiens. Ces dépouilles sont indemnes de toute trace de prélèvement de la viande. Les restes de volaille se rapportent principalement à la poule domestique dont le rôle économique semble relativement faible. Sur l'ensemble du site, la représentation des restes de volaille ne dépasse pas $4 \%$ des restes, et est particulièrement discrète dans les ensembles du "vicus du $\mathrm{I}^{\text {er }} \mathrm{s}$. ", de "Westergass tardif " et du chantier " BK 02-08 ». La représentation de la volaille n'atteint une réelle importance que dans les structures associées au " camp ", au sein desquelles les restes qui lui sont attribués peuvent constituer jusqu'à $12 \%$ de l'ensemble faunique. Les valeurs plus importantes, de $18 \%$ à $45 \%$ des restes, qui caractérisent les dépotoirs les plus récemment fouillés (campagne 2003) sont tout à fait exceptionnelles. L'évaluation de l'importance de la consommation des œufs reste, dans l'état actuel des recherches, hors de notre portée.

\section{LES FRAGMENTS OSSEUX DES TAMISAGES (1999-2002)}

L'étude des restes osseux issus des refus de tamisage a été entreprise afin d'élargir les bases des données relatives à l'alimentation et de cerner plus précisément l'impact des influences romaines dans les provinces septentrionales (tabl. II). D'après les données réunies sur le site de Vindonissa, les premières influences liées à la romanisation sont perceptibles dès les premières décennies du ${ }^{\text {cr }}$ s. apr. J.-C. Celles-ci 
Tabl. II - Décompte des restes osseux de tamisage issus des différents ensembles.

\begin{tabular}{|c|c|c|c|c|}
\hline Espèces (NR) & $\begin{array}{c}\text { " camp " } \\
\text { (4 structures) }\end{array}$ & $\begin{array}{l}\text { "camp/vicus" } \\
\text { (6 structures) }\end{array}$ & $\begin{array}{c}\text { " vicus } \mathrm{I}^{\mathrm{er}} \text { s. apr. J.-C. " } \\
\text { (6 structures) }\end{array}$ & $\begin{array}{l}\text { "vicus II } \mathrm{s} \text { s. apr. J.-C. " } \\
\text { (3 structures) }\end{array}$ \\
\hline Equus sp. & & 1 & 2 & \\
\hline Bos taurus & 1 & 19 & 13 & 1 \\
\hline Ovis aries/Capra hircus & 11 & 26 & 53 & 7 \\
\hline Sus domesticus & 32 & 75 & 89 & 59 \\
\hline Gallus domesticus & 3 & 50 & 60 & 99 \\
\hline Columba domesticus & 5 & 2 & & \\
\hline Cervus elaphus & & & 2 & \\
\hline Lepus europaeus & & & & 5 \\
\hline Talpa europaeus & & & 4 & \\
\hline Arvicula terrestris & & & 2 & \\
\hline Mus musculus & 1 & & 5 & \\
\hline Mammifère & 693 & 260 & 77 & 577 \\
\hline Petit mammifère & 1 & 6 & 8 & 4 \\
\hline Petit carnivore & & & 1 & 2 \\
\hline Erithacus rubecula & & & & 1 \\
\hline Passeriformes & 8 & 2 & 3 & 10 \\
\hline Aves indéterminé & 72 & 28 & 96 & 43 \\
\hline Bufo sp. & & & 1 & \\
\hline Amphibia & & 3 & 85 & 110 \\
\hline Anguilla anguilla & 5 & 3 & 3 & 7 \\
\hline Scomber japonicus & & & 1 & 14 \\
\hline Rutilus rutilus & 2 & & 1 & \\
\hline Thymallus thymallus & 1 & & 3 & 1 \\
\hline Barbus barbus & & & 1 & 2 \\
\hline Leuciscus sp. & 2 & & & \\
\hline Leuciscus cephalus & 5 & & 1 & \\
\hline Salmonidae & 1 & & 1 & 4 \\
\hline Salmo trutta & & & 1 & \\
\hline Perca fluviatilis & 4 & & & 2 \\
\hline Cyprinidae & 51 & & 7 & 9 \\
\hline Alosa alosa & & & & 1 \\
\hline Pisces indéterminé & 71 & 15 & 21 & 21 \\
\hline Bivalvia & 16 & 1 & & \\
\hline Gastropoda & 6 & 133 & 3 & 2 \\
\hline Insecte & 1 & & & \\
\hline Indéterminé & 1087 & 903 & 1947 & 682 \\
\hline Total & 2079 & 1527 & 2489 & 1663 \\
\hline
\end{tabular}

apparaissent assez étroitement liées à l'importation en milieu militaire de denrées issues du monde méditerranéen (maquereaux), tandis qu'une évolution des habitudes alimentaires se reflète aussi à travers la consommation de petits oiseaux (Passeriformes) et la sélection de certains poissons d'eau douce. La question se pose de savoir si de tels comportements peuvent aussi interférer dans la composition des ensembles de faune d'Oedenburg, et si, plus généralement, leur contenu est marqué par l'origine sociale des occupants du site. Les échantillons disponibles pour une telle approche, proviennent des chantiers 01, 04 et 05 des campagnes de fouilles 2001 et 2002 ; ce sont plus précisément les restes des tamis à mailles de $4 \mathrm{~mm}$, complétés par un échantillonnage des restes des tamis à maille de $1 \mathrm{~mm}$ qui ont été pris en compte (fig. 41). Les restes de la fraction inférieure à $0,35 \mathrm{~mm}$ n'ont pas été pris en compte.

L'ensemble " camp » (structures 01-01-365, 02-01-481, 02-01-496, 02-01-533)

Ces échantillons issus de différentes fosses du chantier du " camp militaire " sont tous datés autour de 20-70 apr. J.-C. La composition de certains prélèvements, quatre de la fosse 481 $(\mathrm{NR}=1309)$, l'un de la fosse $496(\mathrm{NR}=278)$ et quatre autres 

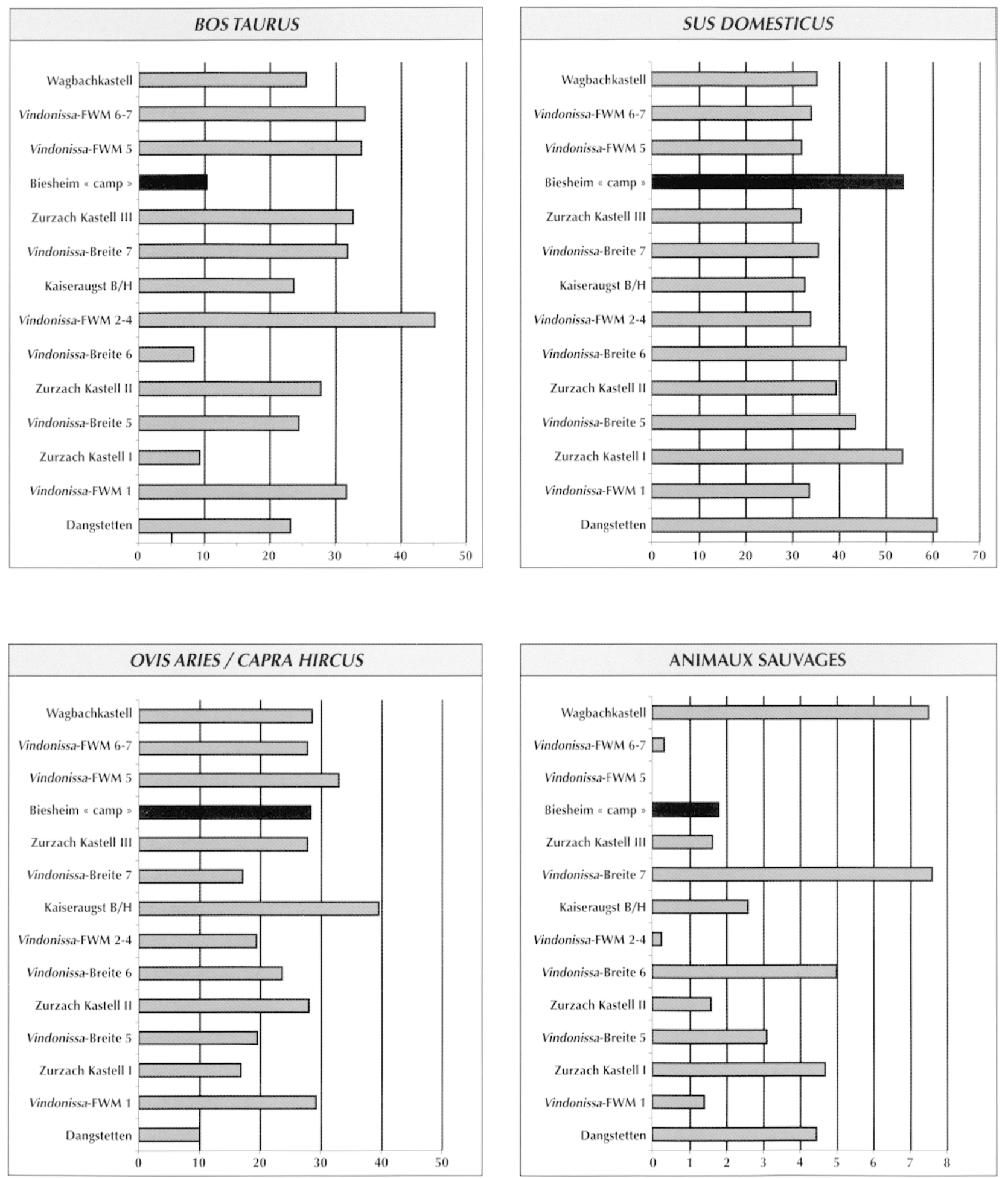

Fig. 41 - Variation de la fréquence des restes des principales espèces dans différents contextes militaires (pourrentages NR). Lees sites somt donnés selon la chronologie de leur occupation de la plus ancienne à la plus récente, de bas en haul. Les ensembles de Biesheim, éludiés dans l'article, sont indiqués en noir et les sites de comparaison sont en grisé : Dangstetten (Uerpmann, 1977); Wagbachkastell (Kokabi, 1987); Kaiseraugst (Deschler-Lrb, 1991); Zurzach (Morel, 1994); Vindonissa-FWM (Ginella et al., 2000); Vindonissa-Breite (Pfäffli, Schibler, 200)3) (DAO F. Ginella, univ. de Bâle). 
de la fosse $533(\mathrm{NR}=458)$, est caractérisée par des proportions importantes d'esquilles digérées (environ $60 \%$ en moyenne). Lne grande partie de ces restes très fragmentés et extrêmement minéralisés n'a pu faire l'objet d'une détermination ostéologique. Le contenu de ces fosses présente toutes les caractéristiques de latrines, ce qu'atteste aussi par ailleurs la découverte de nombreuses pupes de différents insectes. Les restes de mammifères domestiques se rapportent principalement à la poule et au porc. Pour les ossements de pigeon de la fosse 533, une attribution précise à la forme sauvage ou domestique ne peut être avancée. Parmi les ossements de la structure 533 figure aussi un tibiotarse qui n'a pas pu ètre déterminé au rang de l'espèce (Passeriformes). Différentes espèces de cyprinidés, ainsi que l'anguille (Anguilla anguilla), sont les représentants les plus fréquents des ressources halieutiques des cours d'eau environnants. Les associations d'espèces se rapportent plutôt à des étendues d'eau stagnantes ou à faible courant, comme les bras peu actifs du Rhin qui s'étendaient à proximité du camp et du vicus. La taille des poissons consommés varie entre 5 et $80 \mathrm{~cm}$, parmi lesquels la majorité des cyprinidés ne dépasse pas $20 \mathrm{~cm}$. La fosse 365 a également livré des indices de consommation de la perche (Perca fluviatilis) et de la tanche (Thymallus thymallus). Celle de petits oiseaux (Passeriformes) est également bien attestée (sept restes). Ces éléments de faune des refus de tamisage du chantier 01 correspondent à des contenus de latrines. L'alimentation, par ailleurs principalement basée sur le porc et la volaille, qui représentent des denrées plutôt luxueuses, traduit, pour la période romaine, un certain niveau d'aisance. La consommation de petits poissons, de petits oiseaux (Passeriformes) et de pigeons conforte cette observation. Des poissons d'importation ne sont pas attestés dans ces restes de latrines et la représentation de salmonidés, d'ordinaire si appréciés, reste également très discrète.

\section{L'ensemble " camp/vicus " (structures 01-04-08, 01-04-12, 01-04-15, 01-04-18, 01-04-50, 02-04-55)}

Les échantillons des structures $08,12,15,18,50$ et 55 proviennent d'une zone située à l'ouest du canal, à proximité immédiate du camp militaire. Ils sont également datés des années 20-70 apr. J.-C. Les structures 12, 15 et 18 correspondent à des contenus d'urnes (d'après les dernières études, la structure 15 est interprétée comme une fosse-dépotoir), tandis que les structures 50 et 55 se présentent comme des niveaux situés dans des secteurs dans lesquels affleurait la nappe phréatique. Le contenu des échantillons issus des urnes ne présente pas de caractère particulier. Quelques restes isolés d'animaux domestiques y côtoient des vestiges épars d'amphibiens et de micromammifères. La structure 18 se signale par la présence de deux restes de petits oiseaux (Passeriformes), tandis que la structure 50 n'a livré que onze fragments indéterminés. La fosse 08 a livré cinquante-cinq restes déterminables qui proviennent tous, à l'exception de cinq éléments de microfaune, d'animaux domestiques. Plus de la moitié de ces vestiges ont été attribués au porc et parmi les microrestes, deux vertèbres de poisson se rapportent à l'anguille (Anguilla anguilla). La faune de la structure 55 compte 504 restes, constitués pour plus de la moitié par des esquilles d'os de grands mammifères broyés et pour $25 \%$ de restes de petits gastéropodes, indicateurs de milieux humides ou saturés d'eau. Parmi les restes d'animaux domestiques, ceux de porc et de volaille prédominent. D'autres restiges attestent la présence du pigeon.

Parmi les dix-huit restes de poissons, seules trois vertèbres d'anguille ont pu être déterminées jusqu'au rang de l'espèce. Dans l'ensemble, les restes de cet échantillon ne présentent pas de caractéristiques remarquables participant d'une fonction ou d'une origine sociale particulière.

\section{L'ensemble " vicus $\mathrm{I}^{\mathrm{er}}$ s. apr. J.-C. " (structures 01-04-24, 01-04-27, 01-04-73, 02-05-14, 02-05-22, 02-05-140)}

Les échantillons de ces structures représentent des trous de poteaux $(27,73)$ ainsi que différentes fosses. Ces ensembles, datés du I ${ }^{\text {er }}$ s. apr. J.-C., peuvent être attribués au vicus associé au camp militaire. Dans les trous de poteaux ont été recueillis entre 13 et 26 fragments déterminables, qui se rapportent surtout à des animaux domestiques et plus particulièrement au porc. Parmi les restes de microfaune, quatre fragments de petits mammifères ont été reconnus (structure 73) et, au total, sept restes de poissons. Ces derniers attestent la consommation des cyprinidés et de la tanche (Thymallus thymallus). Ce poisson, de la famille des salmonidés (Salmonidae), ainsi que le barbeau (Barbus barbus) parmi les cyprinidés, rendent compte d'une pêche dans un cours d'eau assez important, à fort courant, comme le Rhin par exemple. Par contraste, la composition de la fosse 24 reflète une variété d'espèces beaucoup plus étendue. Les 211 restes d'animaux domestiques se répartissent, en proportions quasiment équilibrées, entre le porc, les petits ruminants et la volaille. Ils représentent au total, parmi les restes issus des refus de tamis à maille inférieure à $4 \mathrm{~mm}$, un peu moins de $80 \%$ des restes déterminés. Les $20 \%$ restants proviennent des espèces les plus diverses telles que le cerf (Cervus elaphus), différentes espèces d'oies, des canards, des petits oiseaux (Passeriformes) et des amphibiens. Parmi les poissons figurent le chevaine (Leuciscus cephalus), la tanche (Thymallus thymallus) et le saumon (Salmo salar). La faune des fosses 14, 22 et 140 s'avère tout aussi riche. Dans ces ensembles a été détecté, entre autres, un reste de maquereau de Méditerranée (fosse 14) ainsi qu'une vertèbre d'anguille (fosse 140). Ces quatre structures contenaient aussi d'importantes quantités de matières fécales (encore non étudiées). Même si, en quantité, ces restes digérés ne présentent pas une représentativité comparable à celle des ossements recueillis dans les fosses du "camp ", leur étude contribue à une restitution plus détaillée de la consommation, en documentant par exemple la consommation des petites espèces de poissons.

\section{L'ensemble " vicus $\mathrm{II}^{\mathrm{e}}$ s. apr. J.-C. " (structures 01-04-38, 02-04-15, 02-04-18)}

Les vestiges disponibles dans le chantier 04 proviennent de deux fosses (38 et 15) et d'un puits (18). La faune de la fosse 38 (fig. 17 i) est représentée par 128 restes recueillis dans les refus de tamisage. Sa composition est marquée par la prédominance, déjà soulignée, des ossements de porc et de volaille, auxquels s'ajoutent aussi des restes de lièrre (Lepus europaeus) et différentes espèces de petits oiseaux (Passeriformes). Parmi les poissons figurent l'anguille (Anguilla anguilla), le barbeau (Barbus barbus), ainsi que des espèces « exotiques " comme la 

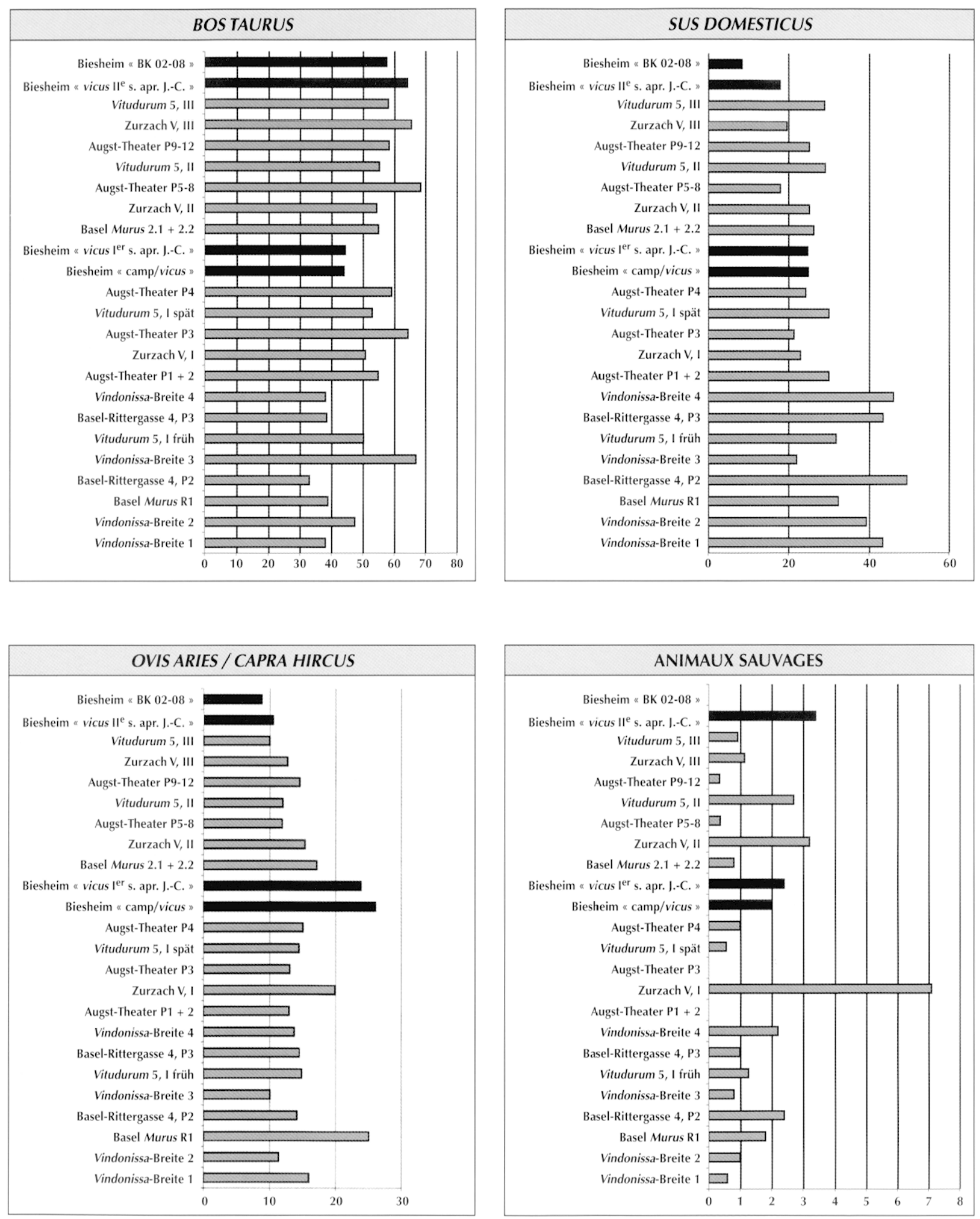

Fig. 42 - Variation de la répartilion des principales espèces domestiques (pourcentages $N R$ ) en contexte civil sur différents sites de comparaison. Les sites sont donnés selon la chronologie de leur occupation de la plus ancienne à la plus récente, de bas en haut. Les ensembles de Biesheim, étudiés dans l'article, sont indiqués en noir et les sites de comparaison sont en grisé: Vitudurum (Morel, 1991); Augst-Théâtre (Furger, Deschler-Erb, 1992); Zurzach (Morel, 1994); Basel Murus (Ebersbach, 1998); Basel Rittergasse (Deschler-Erb et al., 1998); Vindonissa (Pfäffli, Schibler, 2003) (DAO F. Ginella, univ. de Bâle). 
grande alose (Alosa alosa) et le maquereau de Méditerranée (Scomber japonicus). La fosse 15 présente un contenu tout aussi riche. Sur les 133 fragments osseux déterminés jusqu'au rang de l'espèce, l'espèce domestique la plus fréquente est le poulet auquel se rapportent 60 restes. Parmi les espèces de plus faible gabarit, on relève la présence du lièvre (Lepus europaeus) ainsi que de différentes espèces de petits oiseaux (Passeriformes). Les poissons attestés par échantillonnage sont les salmonidés et le maquereau méditerranéen, ainsi que l'anguille et différentes espèces de cyprinidés presque systématiquement attestées dans les refus de tamisage des fosses, même si, dans les fosses 38 et 15 , leur rôle paraît plus discret. Il n'est pour l'instant pas possible de déterminer si la diversité des espèces de poissons, d'oiseaux et de mammifères domestiques est corrélée à l'évolution chronologique du site. C'est un des aspects qui reste à développer dans le cadre des études futures. Dans la fosse 18 ont essentiellement été recueillis, à côté de quelques esquilles de mammifères, des os d'amphibiens qui pourraient correspondre à une accumulation d'origine naturelle.

\section{CONCLUSIONS}

L'étude archéozoologique de ces différents ensembles livre des résultats, dont l'un des principaux intérêts est de fournir une base de comparaison avec les données réunies sur les occupations du plateau et du nord-ouest de la Suisse. Des affinités assez étroites rapprochent plus particulièrement le camp et les installations suisses. Les complexes militaires du $\mathrm{I}^{\text {er }}$ s. apr. J.-C. sont, en règle générale, caractérisés par une faible représentation des bovins, conjuguée à une présence plus importante de restes de petits ruminants, de porc et de gibier. Les ensembles du camp d'Oedenburg reflètent une moindre contribution du bœuf pour une représentation équivalente des petits ruminants, mais une proportion significativement plus importante de restes de porc (fig. 41). Ces différences peuvent s'expliquer par le fait que les effets de l'origine sociale sont encore plus marqués, dans les ensembles du camp militaire d'Oedenburg que dans les sites suisses. Reste à comprendre si cette particularité découle de la sélection des ensembles pris en compte, ou de la présence d'un nombre plus important de militaires de haut rang dans le camp alsacien. Les refus de tamisage provenant du camp et les indices qu'ils livrent de la consommation de petits poissons, de petits oiseaux et de volaille, restituent une alimentation à caractère plutôt luxueux. Les études concernant les secteurs civils du vicus se prêtent aussi assez idéalement à la comparaison avec les données suisses (Schibler, Furger, 1988 ; Lehmann, Breuer, 1997). La seule différence marquante concerne les ensembles du $\mathrm{I}^{\mathrm{er}}$ s. apr. J.-C. dans lesquels les bovins sont moins bien représentés que sur les sites suisses, alors que les fragments de petits ruminants sont plus nombreux (fig. 42). Cela pourrait tenir à une présence et une influence militaires plus importantes qu'ailleurs, sensible jusque dans le vicus, et dont procéderait aussi l'impact des traditions culinaires d'origine méditerranéenne. En témoignent les restes de maquereaux de Méditerranée découverts parmi les reliefs culinaires de chacun des deux ensembles du vicus d'Oedenburg.

J. S., F. G. et H. H. P.

\section{OEDENBURG ET SON ENVIRONNEMENT HISTORIQUE}

Les fouilles trinationales menées à Biesheim et Kunheim permettent de faire progressivement sortir de l'ombre un complexe archéologique majeur pour l'histoire du Rhin supérieur. Son intérêt réside assurément dans la qualité exceptionnelle des macrorestes végétaux conservés en milieu humide, qui font d'ores et déjà d'Oedenburg un site de référence pour le nord des Alpes, dans l'imbrication des problématiques historiques et paléoenvironnementales, mais aussi dans le renouvellement de nos connaissances sur l'occupation humaine de cette région de frontière, à l'époque romaine.

Dans l'état actuel de nos connaissances, la présence d'un habitat groupé à la fin de l'âge du Fer n'est pas prouvée, contrairement à ce qui se passe non loin de là, à Breisach (Weber-Jenisch, 1995). On doit toutefois constater la présence. d'indices sporadiques d'une fréquentation vers le tout début de notre ère : en témoignent des aménagements anthropiques datés de 3-4 apr. J.-C. sous le secteur des temples, et une poignée de tessons tardo-augustéens (timbres d'Ateius, notamment), parfois hors contexte. Mais on n'a pas pour cette époque de témoignage assuré d'une présence militaire romaine, ni d'un habitat indigène structuré. Cette conclusion provisoire pourra naturellement évoluer avec le temps, en fonction de nouvelles découvertes.

En 19 de notre ère, l'un des chenaux qui traversent le site, le Riedgraben, fait l'objet d'un aménagement massif de ses berges. Est-ce là une entreprise qui traduit la présence de l'armée ? On serait tenté de le croire, dans la mesure où le premier établissement militaire ( grand camp») livre actuellement un matériel compatible avec une installation dans les années 20. Il est vrai que cette impression n'est pas fondée sur un grand nombre d'artefacts céramiques, mais l'ensemble monétaire découvert dans le secteur des camps ne s'opposerait pas à une présence de l'armée dès le tout début du règne de Tibère. Dans la mesure où Oedenburg semble avoir été, au $\mathrm{I}^{\mathrm{er}}$ s., dans la mouvance de Vindonissa plutôt que de Strasbourg, l'hypothèse d'une datation précoce devra être confrontée à celle des premiers niveaux du grand camp légionnaire suisse, maintes fois débattue. Les recherches les plus récentes «In der Breite" semblent plutôt indiquer une installation collective de la XIII légion à Windisch vers 14 apr. J.-C., ce qui n'exclut pas la présence possible de soldats, ou d'unités réduites, dès la deuxième décennie avant notre ère (Hagendorn, 1998; Hagendorn et al., 2003). Derrière cette détermination d'une chronologie fine de l'occupation militaire, c'est tout le débat sur le processus de romanisation dans le bassin du Rhin supérieur qui doit être rouvert (Reddé, à paraître).

À l'ombre des soldats se développe une agglomération civile installée dans un milieu marécageux, répulsif, inondable, progressivement aménagé. Plusieurs noyaux d'habitat semblent coexister : aux abords sud-ouest du camp, où les fouilles ont reconnu surtout, dans un premier temps, des zones artisanales ; le long de la via publica, sur une terrasse hors d'eau, mais les niveaux précoces de ce secteur sont pour l'instant mal connus ; enfin, près du Riedgraben, où, vers le milieu du $\mathrm{I}^{\mathrm{er}}$ s., apparaît 


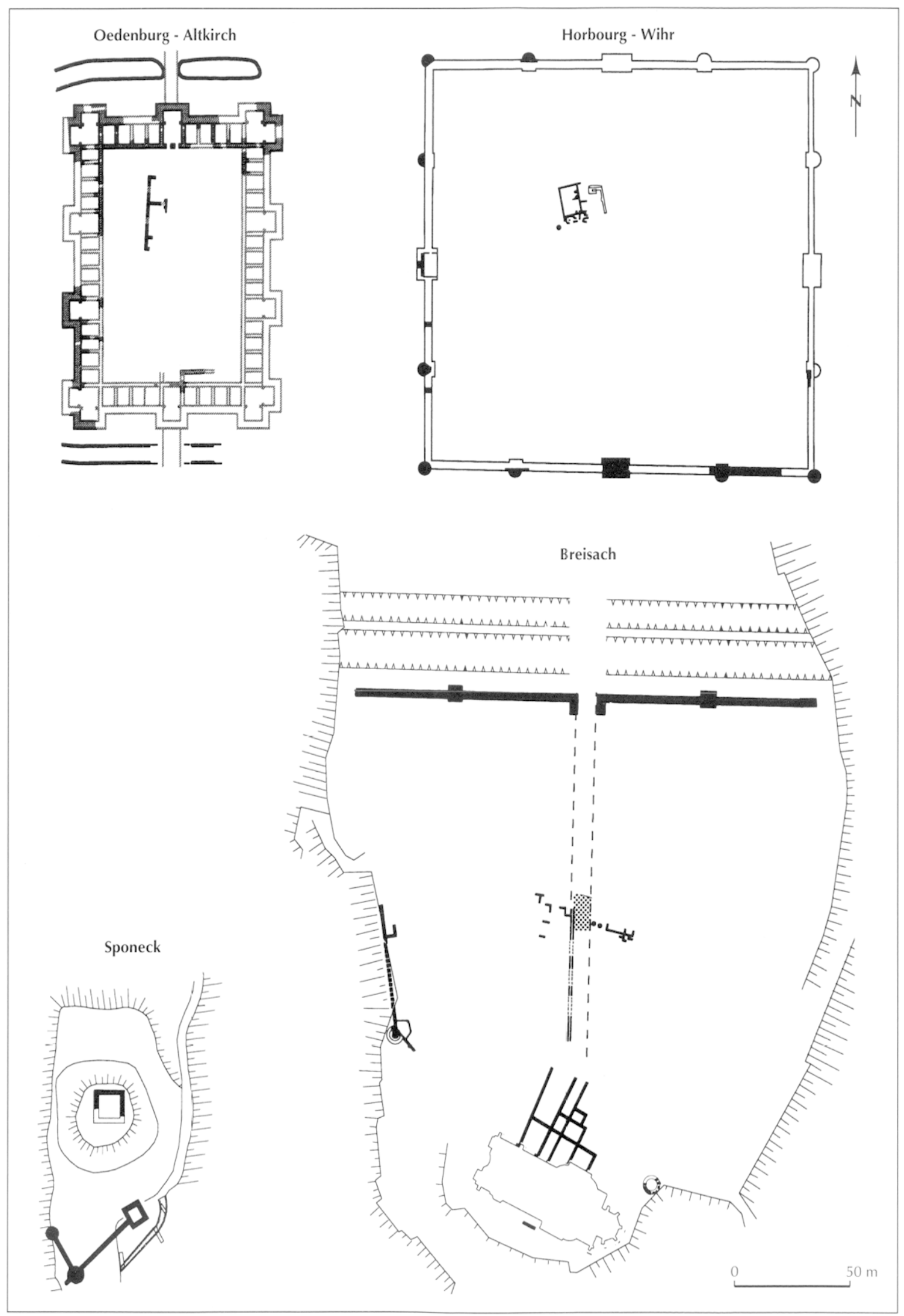

Fig. 43 - Comparaison des dimensions des fortifications du Bas-Lmpire de la région du Kaiserstuhl (dessin S. Berg, univ. de Fribourg-en-Brisgau). 
une zone d'habitat à pans de bois, structurée dans un parcellaire, ainsi qu'un vaste complexe cultuel, de tradition indigène, mais apparemment sans antécédent celtique au vu des connaissances actuelles.

L'ensemble de la plaine de Bade ne reste pas à l'écart de cette phase précoce de la romanisation. R. Asskamp avait déjà montré que la rive droite du Rhin avait été franchie et durablement occupée dès le règne de Claude, en particulier face à Augst et Bâle, mais aussi dans toute la région du Kaiserstuhl (Asskamp, 1989). Les fouilles récentes de la villa d'Heitersheim montrent que les premiers niveaux doivent être datés autour de 30 (Seitz, 2003). Nul doute que le nouveau camp d'Oedenburg n'ait favorisé ce phénomène, que nous ne mesurons malheureusement pas aussi bien sur la rive gauche, en plaine d'Alsace, faute de recherches suffisantes.

Le départ des soldats, vers 69-70, ne se traduit pas par une réoccupation du camp; son emplacement reste à l'écart d'une agglomération qui développe progressivement sa voirie, en continuant d'aménager le sol, abandonnant les zones trop inondables. Malgré ce processus de "pétrification " progressive des constructions, qui voit se développer de grands ensembles à usage public (temples, mansio, thermes, horrea), on ne constate pas le développement d'un centre civique monumental, ce qui fait que le statut juridique de cette agglomération, de plus en plus souvent considérée comme l'Argentovaria de Ptolémée, n'est très probablement pas celui d'une civilas, malgré le terme de $\pi$ o $\lambda$ i $\zeta$ employé par le géographe grec.

Les recherches sur l'Antiquité tardive ont apporté elles aussi leur lot de surprises. Dès 1940, la découverte d'une fortification tardo-antique sur le Münsterberg de Breisach (Nierhaus, 1940) a focalisé l'intérêt sur la région du Kaiserstuhl (Fingerlin, 1979, 1993), l'objectif étant d'étudier le contrôle militaire du Rhin, de l'Iller et du Danube lors des invasions de la fin du $\operatorname{III}^{\mathrm{e}} \mathrm{s}$. (Garbsch, 1970, 1988). Lorsque les fouilles ont été reprises à Breisach à partir de 1966 (Bender éd., 1976), et après la découverte, en 1973, d'une fortification du Bas-Empire au Sponeck (Swoboda, 1986), la question des liens avec Oedenburg a, par la force des choses, été de nouveau posée. La situation avait besoin d'être clarifiée, d'autant plus qu'à la limite ouest de la Forêt-Noire avaient été découvertes et étudiées des fortifications de hauteur des $\mathrm{IV}^{\mathrm{e}}$ et $\mathrm{V}^{\mathrm{e}} \mathrm{s}$.: Zähringer Burgberg, Geisskopf, Kügeleskopf (Fingerlin, 1983; Hoeper, Steuer, 1999 ; Hoeper, 2003). Celles-ci présentaient des caractéristiques germaniques incontestables. Jusqu'aux fouilles récentes de Westergass et d'Altkirch, on ignorait toutefois à peu près tout de l'occupation tardo-antique sur la rive droite du Rhin. Sur diverses cartes, Oedenburg apparaît, en fonction des connaissances de l'époque, soit sans mention caractéristique (Garbsch, 1970), soit comme éventuel lieu de garnison (Bender éd., 1976; Swoboda, 1986), soit encore avec un repère indiquant la présence d'un castrum (Kuhnle, 1991), ou enfin comme "Kastell " (avec un nom erroné) (Hoeper, Steuer, 1999). Le point commun de ces cartes était de mettre en évidence la frontière entre les Romains et les Germains le long du Rhin. Cependant, le fait qu'à partir de l'époque de Dioclétien passait aussi, à cet endroit, la frontière entre les deux provinces romaines de Germania I et de Sequania (Büttner, 1939) n'apparaît sur aucune carte et n'a rencontré aucun écho dans les discussions scientifiq̨ues (fig. 27).

Aussi longtemps que n'étaient pas connues les dimensions et la datation de la fortification d'Oedenburg, il n'était pas possible de juger de la situation militaire entre les Vosges et la Forêt-Noire à la hauteur du Kaiserstuhl. Ceci n'était pas seulement valable pour l'identification d'Argentovaria/ Argentaria à Horbourg, mais surtout pour la détermination du rôle de Breisach. La fortification d'Oedenburg formait-elle une base sur la rive gauche du Rhin et Breisach était-elle la tête de pont sur la rive droite du Rhin, au même titre qu'Augst/ Wyhlen ou Basel/Kleinbasel, ou bien Breisach était-elle réellement située sur une île du Rhin, comme l'indique une source écrite du $\mathrm{X}^{\mathrm{e}}$ s. (Haselier, 1969, p. 58 sqq.)?

Avec la détermination du plan de la fortification d'Oedenburg, il est à présent possible de définir non seulement sa forme, mais surtout son rôle par rapport aux autres fortifications de ce secteur géographique (fig. 43). Sa superficie, légèrement supérieure à 1 ha, la place derrière Brisiacum/Breisach (3,5 ha) ou Horbourg-Wihr (2,5 ha), mais nettement devant Sponeck $(0,3$ ha).

En liaison avec Oedenburg, Breisach occupe une position unique au sein de toutes les fortifications tardives établies le long du Rhin. Celle-ci se distingue nettement du schéma traditionnel, caractérisé par les grandes bases sur la rive gauche (Mayence, Cologne) et, de l'autre côté du fleuve, les petites têtes de pont (Mayence-Kastel, Deutz). Breisach était le grand centre, Oedenburg la base de pont plus petite située sur la rive gauche; sur la rive droite, la garnison de Sponeck pouvait observer et faire barrage vers le nord. Depuis que l'on a découvert des objets du Bas-Empire à Riegel, parmi lesquels une estampille de la Legio I Martia (Dreier, 1999), on peut aussi envisager la présence d'un poste militaire au sud dans la région d'Ihringen, d'où provient par ailleurs une partie des pierres utilisées pour la construction de la fortification d'Oedenburg. À cela il faut ajouter désormais les sites de hauteur, à la lisière de la Forêt-Noire; ceux-ci, à notre sens, ont fait partie d'un concept d'ensemble destiné à surveiller la plaine du Rhin au sud de la limite entre les provinces de Germania Prima et de Sequania.

Pourtant, même après six années de recherches nous ne savons pas à quel moment l'armée romaine a repris garnison à Oedenburg (avant, pendant ou après l'époque de Dioclétien et avec quels effectifs ?). Les deux constructions du Bas-Empire étudiées ces dernières années ont certes un lien avec l'organisation militaire, mais ceci reste à définir. Aussi bien le praetorium postconstantinien de "Westergass" que la fortification valentinienne d'" Altkirch " occupaient une situation identique par rapport aux voies de communication. Elles sont en relation avec la via publica sud-nord (située immédiatement à l'ouest), à laquelle elles sont tangentes, ou à laquelle elles sont directement raccordées. Définir cette organisation militaire tardoantique à l'échelle régionale constituera assurément, dans les années à venir, l'un des principaux objectifs de la recherche.

H. L. N. et M. R. 


\section{ANNEXE}

\section{LA FORTERESSE TARDIVE SUR UNE CARTE INÉDITE D'ANTOINE CESTRE}

Jusqu'à présent, un certain nombre de sources anciennes permettaient d'appréhender partiellement l'état du site galloromain d'Oedenburg tel qu'il se présentait au XIX ${ }^{\mathrm{e}}$ s., mais les données étaient très lacunaires. La découverte toute récente d'un manuscrit inédit d'Antoine Cestre (1823-1899, né et mort à Neuf-Brisach, conducteur des travaux des Ponts et Chaussées) permet une nouvelle approche. Ce manuscrit est formé d'un recueil d'articles sur la région de Neuf-Brisach, dont deux sont consacrés au site d'Oedenburg. Une très intéressante carte du secteur, à l'encre, accompagne ce manuscrit (fig. 44) ; elle montre l'état du fleuve au XIX ${ }^{\mathrm{e}} \mathrm{s}$. avec ses nombreuses îles et le niveau de l'inondation de 1852. L'étude de ces documents inédits et la relecture des autres sources contemporaines permettent de réévaluer la chronologie des épierrements successifs du site antique et de préciser son état de conservation au XIX ${ }^{\mathrm{e}} \mathrm{s}$.

Plutôt qu'à la forteresse de Neuf-Brisach, il semble que la première cause d'épierrement du site antique doit plutôt être rapportée à la construction de La Villeneuve-lès-Brisach (ou La Villeneuve-Saint-Louis), ville dont la création ex nihilo a été décidée en 1674 par Louis XIV pour abriter le Conseil souverain d'Alsace (Wilsdorf-Collet, 1998). Installée sur une île face à Breisach, cette ville, capitale judiciaire de l'Alsace, est dotée d'une fortification puissante dont les plans sont dressés par Vauban ; un fort, une église, le couvent des Récollets, des bâtiments publics et le palais du Conseil souverain nécessitèrent aussi une grande quantité de pierres de taille, et l'on peut supposer que le site gallo-romain d'Oedenburg, très proche, a dû être exploité dès cette époque. En 1697, par le traité de Ryswick, le Rhin redevient frontière ; la démolition de La Villeneuve est imposée car elle pourrait servir de tête de pont face à Breisach. L'existence de cette ville fut donc éphémère et " les matériaux provenant de la démolition des maisons de la ville de Saint-Louis "serviront en partie à la construction de la forteresse (Coste, 1860). À cette occasion, le site antique d'Oedenburg a sans doute aussi servi à nouveau de carrière, mais pas de manière exclusive, ni même exhaustive, comme on le considère généralement.

Les épierrements du XVII ${ }^{\mathrm{e}} \mathrm{s}$. n'ont en effet pas réussi à faire disparaître totalement les murs de la cité antique. Plusieurs historiens du XIX ${ }^{e}$ s. rapportent que des ruines assez considérables étaient encore visibles à cette époque. En 1789, une

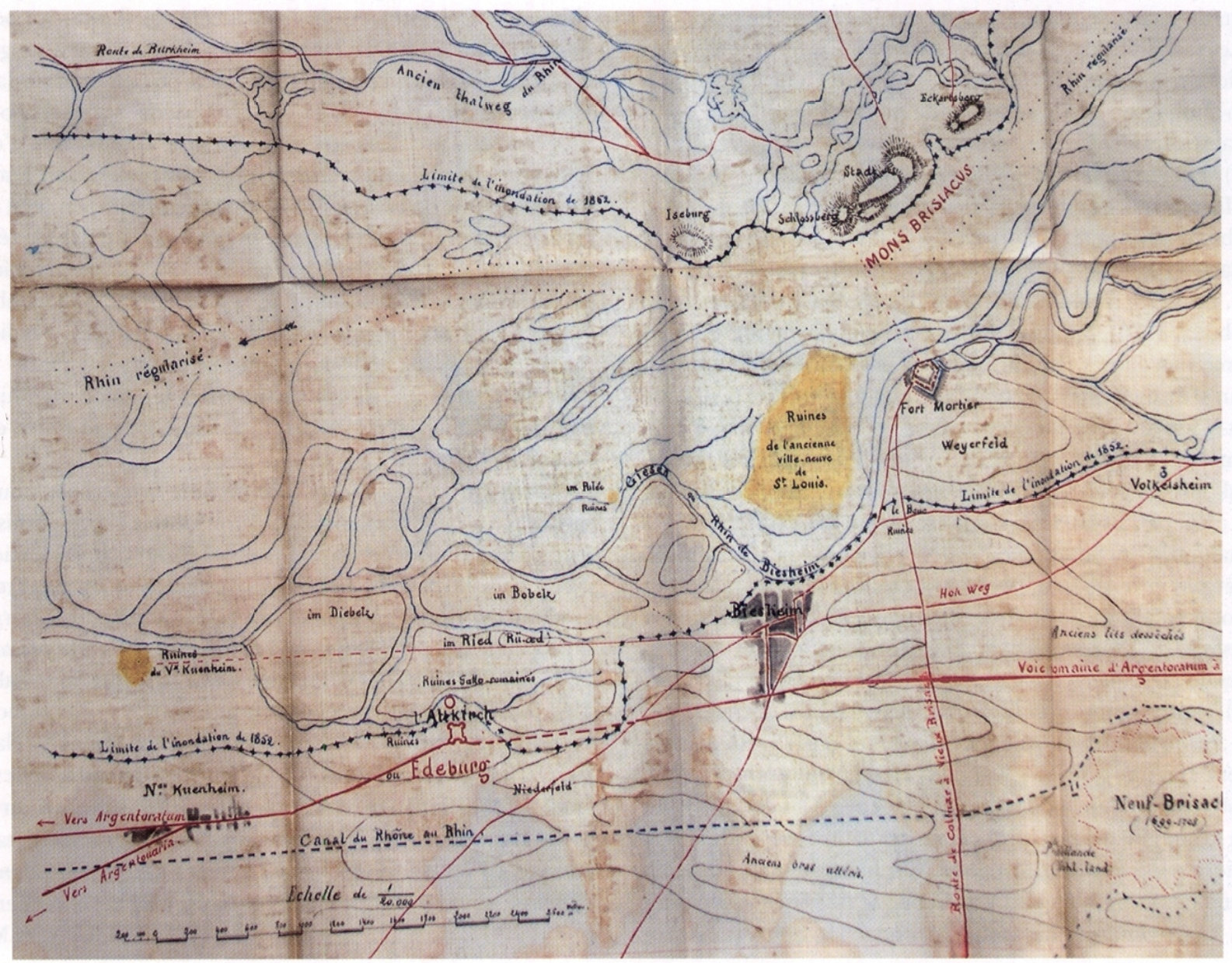

Fig. 44 - Le site d'Oedenburg au XIX ${ }^{e}$ s., sur une carte inédite d'Antoine Cestre. 
première publication signale les plus anciennes découvertes (un bas-relief de qualité remarquable et deux «briques » estampillées de la XXI" légion); elle apporte aussi des informations précises sur l'état du site à la fin du XIIII' s., affirmant que "les ruines [...] s'y voient encore, malgré la grande quantité de pierres qu'on n'a cessé d'en enlever depuis longtemps "; elle insiste enfin sur son étendue car les ruines " démontrent que cet endroit s'étendait depuis la route du Rhin jusqu'à la rive occidentale de ce fleuve" (Morel, 1789). Plusieurs autres sources confirment la présence de murs encore visibles. Philippe de Golbéry parle de "ruines [...] où l'on déterre de vastes fondations " (Golbéry, 1828). Un peu plus tard, Alphonse Coste rapporte que des fouilles réalisées sur la butte d'Altkirch en 1822, " eurent pour résultat d'amener au jour [...] des portions de murailles romaines d'une assez grande étendue " (Coste, 1858). En 1868, l'architecte colmarien Dietrich parle de l'existence d'" une cité entière " avec des assises en pierres et des vestiges sur une étendue de $3 \mathrm{~km}$, oì " des terrains mouvementés et ondulés font pressentir de nombreuses substructions" (Dietrich, 1868).

C'est cependant incontestablement à Antoine Cestre, passionné par l'archéologie locale, que nous devons les informations les plus intéressantes sur l'état du site, qu'il connaît bien puisqu'il participe aux travaux réalisés en 1869 pour la construction d'un canal de dérivation entre le Rhin et le canal du Rhône-au-Rhin. À propos des fouilles réalisées en 1822, il parle de "substructions dont le développement s'étendait jusque sous la route nationale " (Cestre, 1877). Surtout, il est le seul à signaler vraiment l'existence d'un castrum, que P. de Golbéry n'avait évoqué que par l'étymologie du mot Oedenburg, qui signifie "château détruit" (Cestre, 1884). À plusieurs reprises, il donne des informations sur l'existence de ce castrum et sur sa localisation précise. Dans une notice du manuscrit inédit, datée de 1877 , il écrit qu'Oedenburg " était situé à l'emplacement d'un petit poste romain dont les ruines se remarquaient encore en 1876, à l'extrémité nord de la banlieue de Biesheim au lieu-dit : Altkirch [...] à l'endroit où au Moven Âge, s'élevait l'église d'Oedeburg "; il ajoute encore : "Ici comme en maintes localités, les fondations du castrum auront servi à bâtir l'église ". Précisant toutes ces informations, la carte colorisée jointe au manuscrit constitue un élément fondamental à ce dossier. Elle indique l'emplacement précis du castrum, le long de la route allant de Biesheim à Kunheim. De toute évidence, ce castrum dont les ruines étaient encore visibles correspond à la forteresse-palais d'époque valentinienne fouillée par l'équipe allemande sous la direction de H. U. Nuber et G. Seitz.

S. P. 


\section{LISTE DES AUTEURS}

GINEI.l A Francesca, Institut für Prähistorische und Naturwissenschaftliche Archäologie, Archäobiologie, Universität Basel, Spalenring 145, CH-4055 Bâle. Courriel : francesca.ginella@stud.unibas.ch

GISSINGER Bastien, 37 rue Saint-Denis, F-92700 Colombes. Courriel : trajan@tiscali.fr

HüSTER PI.ogMANN Heidemarie, Institut für Prähistorische und Naturwissenschaftliche Archäologie, Archäobiologie, Universität Basel, Spalenring 145, CH-4055 Bâle. Courriel : heide.huester-plogmann@unibas.ch

JACOMET Stefanie, Institut für Prähistorische und Naturwissenschaftliche Archäologie, Archäobiologic, Universität Basel, Spalenring 145, CH-4055 Bâle. Courriel : stefanie.jacomet@unibas.ch

JOLY Martine, Institut d'art et d'archéologie, Université de Paris-IV, 3 rue Michelet, F-75006 Paris.

Courriel : martine.joly8@wanadoo.fr

NCBER Hans Llrich, Provinzialrömische Archäologie, Albert-Ludwigs-Universität, Glacisweg 7 ,

D-79085 Fribourg-en-Brisgau. Courriel : provroem@uni-freiburg.de

OL.r.mE Vincent, Centre des sciences de la terre, Faculté des sciences, Université de Dijon, 6 boulevard Gabriel,

F-21000 Dijon. Courriel : vincent.ollive@u-bourgogne.fr

PEL.I.ISSIER Julien, Antea, 29 rue Gilardoni, F-68190 Altkirch.

PETIT Christophe, Centre des sciences de la terre, Faculté des sciences, Université de Dijon, 6 boulevard Gabriel,

F-21000 Dijon. Courriel : christophe.petiı@u-bourgogne.fr

PLouin Suzanne, Musée de Biesheim, F-68600 Biesheim. Courricl : splouin@wanadoo.fr

Popovtrch Laurent, Centre des sciences de la terre, Faculté des sciences, Lniversité de Dijon, 6 boulevard Gabriel,

F-21000 Dijon. Courricl : laurent.popovitch@wanadoo.fr

RFDDÉ Michel, École pratique des hautes études (IV" section), 45 rue des Écoles, F-75005 Paris.

Courricl : redde.michel@club-internet.fr

SCHIBI.ER Jörg, Institut für Prähistorische und Naturwissenschaftliche Archäologie, Archäobiologie,

Universität Basel, Spalenring 145, CH-405ว Bâle. Courriel : joerg.schibler@unibas.ch

SCHLUMBACM Angela, Institut für Prähistorische und Naturwissenschaftliche Archäologie, Archäobiologie,

Universität Basel, Spalenring 145, CH-4055 Bâle. Courriel : angela.schlumbaum@unibas.ch

SCHLCANY Caty, Institut für Prähistorische und Naturwissenschaftliche Archäologie, Universität Basel, Spalenring 145,

CH-4055 Bâle. Courriel : caty.schucany@bluewin.ch

SCHWARZ Peter-Andrew, Institut für Prähistorische und Naturwissenschaftliche Archäologie, Universität Basel,

Spalenring 145, CH-4055 Bâle. Courriel : peter-andrew.schwarz@unibas.ch

SEIT\% Gabriele, Provinzialrömische Archäologie, Albert-Ludwigs-Universität, Glacisweg 7, D-7908s Fribourg-en-Brisgau. Courricl : provroem@uni-freiburg.de

VANDORPl Patricia, Institut für Prähistorische und Naturwissenschaftliche Archäologie, Archäobiologie, Lniversität Basel, Spalenring 145, CH-4055 Bâle. Courriel : patricia. vandorpe@unibas.ch

VIROLlEY Bénédicte, Service départemental archéologique du Haut-Rhin, 15 place de l'Église, F-68190 Ensisheim.

Courriel : viroulet.archeo.sdatis@ewanadoo.fi

WICK Lucia, Institut für Prähistorische und Naturwissenschaftliche Archäologie, Archäobiologie,

Universität Basel, Spalenring 145, CH-4055 Bâle. Courriel : lucia.wick@unibas.ch

Wol.F Jean-Jacques, Service départemental archéologique du Haut-Rhin, 15 place de l'F́glise, F-68190) F.nsisheim.

Courriel : wolf.archeo.sda68@wanadoo.fr 


\title{
BIBLIOGRAPHIE
}

\author{
Abréviations \\ DAF Documents d'archéologic française. \\ RIC The Roman Imperial Coinage. \\ $R G A$ Reallexikon der Germanischen Alterlumskunde.
}

\section{ALTJOHANN M.}

1995 : "Bemerkungen zum Lrsprung des gallo-römischen Lingangstempels ", in Cass W., Hëssex (. . M., KLINF.. H.-P., SOMMER (. S., WEBER G. (E.DS), Provinzialrömische Forschungen. Festschrift für Günter Ulbert zum 65. Ceburtstag, Espelkamp, Marie Leidorf, p. 169-203.

\section{ANDRÉ J.}

1998 : Essen und Trinken im alten Rom, Stuttgart, Reclam, 279 p., 107 fig.

\section{ANTHES E.}

1918 : "Spätrömische Kastelle und feste Städte im Rhein- und Donaugebiet ", Bericht der Römisch-Germanischen Kommission, 10, p. 86-165.

\section{ASSKAMP R.}

1989 : Das südliche Oberrheingebiet in frührömischer Zeit, Stuttgart, Theiss (coll. Forschungen und Berichte zur Vor- und Frühgeschichte in Baden-Württemberg, $33), 123 \mathrm{p}$.

\section{BAAS J.}

1982 : " Kultur- und Nutzpflanzen aus römischen Siedlungsgruben in NidderauHeldenbergen (Mainz-Kinzig-Kreis). Ein Beitrag zur Geschichte unserer Kulturpflanzen ", Saalburg-Jahrbuch, 38, p. 110-119.

1987 : « Römer»citliche Kultur- und Nutzpflanzen aus Windisch, Arenches und Cham in der Schwei\% ", SaalburgJahrbuch, 43, p. 108-111.

1990 : " Bedeutende römerzeitliche Obstfunde in den Kantonen Waadt, Zug und Aargau ", Helvetia Archaeologica, 21, p. $28-31$.

\section{BAKELS C. C., JACOMET S.}

2002 : " Luxury Foods in Roman Period Central Europe. Contributions from Archaeobotany ", in VAN DER VEEN M. (ED.), "L Luxury Foods ", World Archaeology, 34 , p. $542-557$.

\section{BaUMaNN M., BabotaI C., SChibleR J.}

2005 : Native or Naturalized? Validation of Lngulate Distribution Models with Arrharozoological Data, Ecological Applications, vol. 15, n"3, p. 1096-1110.

\section{BECHERT T.}

1971 : " Römische Lagertore und ihre Bauinschriften. Ein Beitrag zur Entwicklung und Datierung kaiserzeitlicher Lagertorgrundrisse von Claudius bis Severus Alexander ", Bonner Jahrbücher, 171, p. 201-287.

BECK H., GEUenich D., STEUeR D. H.

2003: RGA, 23, Berlin, New York, De Gruyter, $640 \mathrm{p}$.

\section{BENDER H.}

1976 : "Neuere Untersuchungen auf dem Münsterberg in Breisach. Die Ausgrabungen in den Jahren 1966-1975 ", Archäologisches Korrespondenzblatt, 6 , p. 213-224 et p. 309-320.

\section{BENDER H. (ÉD.)}

1996 : Das "Bürgle " bei Gundremmingen. Die Crabung 1971 und neue Funde, Passauer Universitätsschriften zur Archäologie, Espelkamp, Marie Leidorf (coll. Passauer Universitätsschriften zur Archäologie, 3), $176 \mathrm{p}$.

\section{BERNHARD H.}

1982 : " Germanische Funde der Spätantike zwischen Straßburg und Mainz ", SaalburgJahrbuch, 38, p. 72-109.

\section{BIELLMANN P.}

1987 : "Les tuiles de la le légion Martia trouvées à Biesheim-Oedenburg ", Annuaire de la Société d'histoire de la Hardt el du Riel, 2, p. 8-15.

1988 : "Biesheim-Oedenburg: un bimillénaire ", Annuaire de la Société d'histoire de la Hardt el du Ried, 3, p. 21-30.

1996 : « Le premier camp d'Oedenburg (Biesheim-Kunheim) ", Annuaire de la Société d'histoire de la Hardt et du Ried, 9, p. 17-32.

1997 : " Obscrvations sur l'occupation romaine tardive du site d'Oedenburg (Biesheim-Kunheim) ", Annuaire de la Société d'histoire de la Hardt et du Ried, 10, p. $17-38$.

2000 : «I a prospection pédestre à Oedenburg (Biesheim-Kunheim) en 1999 ", Anmuaire de la Société d'histoire de la Hardt et du Ried, 13 , p. 18-22.

2001 : "Lne nécropole du Bas-Empire à Oedenburg (Biesheim-Kunheim "), Anmuaire de la Société d'histoire de la Hardt et du Ried, 14, p. 9-20.
Blanalt J.-G., Eller J.-P., Fluck P., GEFFROY J., HILLERMANN G., JEHL M., MONNET C., SHREINER J., ECKERLE A., SitTler C., SCHWOERER P., Tricart J., HiRTh C., Vogt M., Weil R., Siat A. 1974 : Notice explicative de la carte géologique de la France au 1/50 000, Feuille ColmarArtolsheim.

\section{BÖHME H. W.}

1974 : Germanische Grabfunde des 4. bis 5. Jahrhunderts zzwischen unterer tilbe und Loire, Münich, C. H. Beck (coll. Münchner Beiträge zur Vor- und Frühgeschichte, 19), 2 vol., 384 p. et 147 ill.

1998 : "Franken und Romanen im Spiegel spätrömischer Grabfunde im nördlichen Gallien ", in (GELENICH D. (F́D.), Die Franken und die Alemannen bis zur ". Schlacht bei Zülpich " (496/97), Berlin, New York, De Gruyter (coll. Suppl. au R(i.t, 19), p. $31-58$.

BRem H., STeiner D., KesSElRing R.

1999 : " Neues aus Tasgetium ", Archäologie der Schweiz, 22, p. 123-133.

BRISTOW C. S., BEST J. L.

1993 : « Braided Rivers: Perspectives and Problems ", in BEST J. L.., BRISTOH C. S. (EDS), Braided Rivers, London, Geological Society (coll. Geological Society of London, Special Publication, 75), p. 1-11.

\section{BROMBACHER C.}

1995 : "Archäobotanische Lntersuchungen ron Getreideproben aus dem römischen Vicus Basel Rittergasse ", in HFI.MIG G., SCHÖN U. (ÉDS), "Neuc Befunde zur antiken Zufahrtsstrasse auf den Basler Münsterhügel ", Jahresbericht der Archäologischen Bodenforschung des Kantons Basel-Stadt, Basel, Kanton Basel-Stadt, p. 55-56.

BURNHAM B. C., WACHER J.

1990 : The Small Touns of Roman Britain. Londres, Batsford, $388 \mathrm{p}$.

BÜTTNER H.

1939: Geschichte des Flsaß I- Politische (jeschichte des Landes yon der Landnahmezit bis zum Tode Ottos III, Berlin, Juner und Dünnhaupt (coll. Neue deutsche Forschungen, Abteilung Mittelalterliche Geschichte, 8: veue deutsche 
Forschungen, 242) [nouvelle édition : 1990, Sigmaringen, Jan Thorbecke], $224 \mathrm{p}$.

\section{CARBEINER R.}

1969 : "L.e Grand Ried d'Alsace ", in "Le Ried Centre Alsace ", Bulletin de la Société industrielle de Mulhouse, 734, p. 15-44.

\section{Cestre A.}

1877: Histoire et géographie ancienne. Varia. Notice sur Oedeburg, manuscrit non paginé. 1884 : Ruines d'Oedeburg sur le Rhin (banlieue de Biesheim), Musée de la ville d'Altkirch, Altkirch, Boehrer, $33 \mathrm{p}$.

\section{CHRISTLEIN R.}

1974 : "Grabfunde des 5. Jahrhunderts von Frickingen, Ortsteil Bruckfelden, Kreis Überlingen ", Fundberichte aus BadenWürttemberg, 1, p. 565-572.

\section{Church M.}

1978 : "Paleohydrological Reconstruction from a Holocene Valley Fill ", in MLAL.L. A. D. (ED.) Fluvial Sedimentology, Calgary; Canadian Society of Petroleum (coll. Canadian Societs of Petroleum, (icologists Memoir, 5), p. 743-772.

\section{CONRAD O.}

1999: "Fléaux et calamités naturclles au siecle dernier dans te Haut-Rhin ". Annuaire de la Société d'histoire do la Hardt et du Riel, 12, p. 115-136.

\section{Coste A.}

1858: "Argentouaria, station gallo-romaine de l'Alsace ", Rerue d'Alsace, 9, p. 87-95. 1860) : Notice historique et topographique de la ville de Vieux-Brisach, avec le plan de la ville en 1692, Mulhouse, P. Risler; 272 p., 1 carte.

CÜNTZ O. (ÉD.)

1990 : Itineraria Antonini Augusti et Burdigalense, Sutugart, Teubner (coll. Itineraria romana, 1), 2 vol.

\section{CÜPPERS H.}

1965: "Palatiolum-Pfalzel ", in REtesch W. (EDD.), Frühchristliche Zengraisse im Einzugsgebiet von Rhein und Mosel, Trier, Lnitas Buchhandlung. p. 152-162.

1984 : "Palatiolum-Pfalzel. Palastburg und Kaseme ", in RHIISISCHES LANDESMISLEM TRIER (IID), Ausstellungskatalog Trier: Kaiserresidenz und Bischofssitz. Die Stadt in spätantiker und frühchristlicher Zeit, Mainz, Philipp von Zabern, p. 319-322.

1990 : " Trier-Pfalzel ", in (C̈PPERs H1. (1:D).), Dip Rïmer in Rheinlunt-Pfuls, Stuttgart, Theiss, p. 649-653.

\section{DE HINGH A.}

1993 : "Bottle gourd seeds at Gallo-Roman "Le Bois Harlé" (Oise, France) ", Analecta Prathistorica Leidensia, 26, p. 93-97.

\section{DEGEN R}

1963 : " Fabrikmarken römischer Privatzicgeleien in der Schweiz ", $\mathrm{Cr}$ Schweiz, 27, p. 33-38.

\section{DESCHLER-ERB S.}

1991 : "Auswertung von Tierknochenfunden der Grabung Kaiseraugst-Bireten/ "Haberl" 1979.01. Speise- und Schlachtabfälle römischer Truppen in der Kaiseraugster Unterstadt der 1. Hälfte des 1. Jahrhunderts ", in DFSCHLER-ERB E., PLTER M., DESCHI.ER-ERb S. (ÉDS), Das frühkaiserzeitliche Militärlager in der Kaiseraugsler Unterstadl, Augst, Amt für Museen und Archäologie des Kantons Basel-Land (coll. Forschungen in Augst, 12), p. 121-131.

1999 : “Vom Packesel zum Kurierpferd Archäozoologische Aussagemöglichkeiten zu den Equiden in römischer Zeit ", in KriMkES M., SCHELERBRAND'T J. (ÉDS), Fragen zur römischen Reilerei, Kolloquium zur Ausstellung "Reiter wie Statuen aus Erz. Die römische Reiterei am Limes zuischen Patrouille und Parade ", Stuttgart, Limesmuseum Aalen, p. 64-71.

\section{Deschler-ERB S., SCHIBLeR J., HÜSTER} PlogmanN H.

2002 : "Vichzucht, Jagd und Fischfang ", in Flctisch l., Niffel fer l., Rossi F. (ÉdS), Die Schweiz vom Palüolithikum bis zum frühen Mittelalter: Römische Zeit, La Suisse du Paléolithique à l'aube du Moyen Âge -5Époque romaine, Bâle, Société suisse de préhistoire et d'archéologie, p. 165-171.

DESCHLER-ERB S., SCHIbleR J., VESZELI M. 1998 : "Überlegungen zur spätlatènezeitlichen und augusteischen Epoche aus archäozoologischer Sicht: Auswertung der Tierknochenfunde aus der Gabung Bascl-Rittergasse 4 (1982/6) ", in HF.(AIT Y. (E.D.).), " Dic Ausgrabungen auf dem Basler Münsterhügel an der Rittergasse 4 ", Materialhefle zur Arwhöologie in Basel, 16, p. 154-179.

\section{DICK M.}

1989: "Verkohlte Samen und Früchte aus awci holzkohlereichen Schichten ron Augst (Augusta Rauricorum ; Forum und Insula 23) ", Jahresberichte ans Augst und Kaiserangst, 10, p. 347-350).

\section{DIETRICH J. J.}

1868: "Rapport sur les Antiquités trowées aux environs de Colmar ", Bulletin de la Société pour la conservation des Manuments historiques d'Alsace, 2" sćric, 6, p. 93-101.

\section{DRACK W.}

1980 : Die spälrömische Grenzwehr am Hockrhein, Zürich, Schweizerische Gesellschaft für Lr- und Frühgeschichte (coll. Archäologische Führer der Schweiz, 13), 48 p.

\section{DREIER C.}

1999 : "Zwei spätantike Neufunde aus Riegel a. K., Kr. Emmendingen. Hinweise auf einen Militärposten?" Fundberichte aus Baden-Württemberg, 23, p. 253-259.

\section{EBERSBACH R.}

1998 : "Ausgrabungen am Basler Murus Gallicus 1990-1993 -2- Die Tierknochen, mit einer vergleichenden Auswertung der Tierknochen aus der Alten Landvogtei in Riehen (BS) und aus dem "Hebammenhaus" in Kaisten (AG) ", Materialhefle zur Archäulugie in Basel, 13, p. 12 et 116.

\section{EBERSBACH R., SCHRÖDER S.}

1997 : « Roman Occupation and its Economic Consequences in the Northern Part of Switzerland ", in VIGNE J.-1). (DIR.), "L.Homme et l'animal, Proceedings of the $7^{\text {th }}$ conference, Konstan\%, 26 sept.$\mathrm{I}^{\mathrm{er}}$ oct. 1994 ", Anthropozoologica, 25-26, p. $449-456$.

\section{EGGER R.}

1966 : Das Praetorium als Amtssitz und Quartier römischer Spitzenfunklionäre. Wien, Österreichische Akademie der Wissenschaften, Sitzungsberichte 250 , p. 3-47.

\section{FAHR R., ReICHMANN C.}

2002 : " Dic Kasernen des Kastells Gelduba (Krefeld-(;ellep) in frühflavischer Zeit. Überlegungen zur Garnison ", Germania, 80,2 , p. $475-489$.

\section{FAUDUET I.}

1993a: Allas des sanctuaires romano-celticues de Gaule: les fanums, Paris, éd. Errance, $140 \mathrm{p}$.

1993b: Les Temples de tradition celtique on Gicule romaine, Paris, éd. Errance, $159 \mathrm{p}$.

\section{FEIGENWINTER F.}

1997 : "Die Pflanzenfunde aus der Latrine", in JACСH V. (F́D), " Fschenz-Tasgetium. Römische Abwasserkanäle und Latrinen ", Archäologie im Thurgau, 5, p. $21-28$.

\section{FELLManN R.}

1988 : "Militär- und Privatyicgeleien ", in DRACK W., FELLALN R. (ÉDS), Dit Römer in der Schuepiz, p. 203, fig. 172.

1991 : "Hölzcrne Kleinfunde aus dem Vicus Vitudurum-Oberwinterthur ", in 
ETTER H. F, FEIIMAAN BROGil. R., FFIIMANX R., MARTIN-KII.CHFR S., MORHI. P., RISI A. (ÉDS), Beiträge zum römischen Oberwinterthur-Vitudurum $;$ Zürich, Berichte der Zürcher Denkmalpflegc (coll. Archäologische Monographien, 10), p. 17-39.

1995 : " Germania superior, in der Städte sind... von den Raurikern aber Augusta Raurikon und Argentovaria. Kritische Bemerkungen zu civitas und colonia im Raurikergebict ", in KOE.NI; F., REBETEz S. (FDS), Arculiana Festschrift Hans Bögli, Avenches, L.A.O.T.T., p. $289-301$

\section{FELLMANN R., WOLF J.J.}

1993 : "Note sur le Practorium de KembsNeuweg 1991 ", in "Mćlanges offerts à Jean-Jacques Hatt ", Cahiers alsaciens d'archéologie, d'art el d'histoire, 38, p. 112-114.

\section{FEUGÈRE M.}

1993 : Les Armes des Romains de la République à lAntiquile tardive. Paris, éd. Errance. $287 \mathrm{p}$

\section{FINGERLIN G.}

1979 : " Kastellorte und Römerstraßen im frühmittelalterlichen Siedlungsbild des Kaiserstuhls ", in WER.ER J., EWIG E. (EDS), Von der Spätantike zum Mittelalter Sigmaringen, Thorbeke (coll. Vorträge und Forschungen, 24), p. 379-409.

1983 : " Der Zähringer Burgberg, eine neuentdeckte Höhensiedlung der Volkerwanderungs\%eit, Gemeinde Gundelfingen, Kreis BreisgauHochschwarswald ", Archäologische Ausgrabungen in Baden-Württemberg, 1983, p. 181-184.

1986 : " Breisach, Spätrömisches Grenzkastell ", in FILTZIN(;i:R P., Planck D., Caimmirler B. (ÉdS), Die Römer in Baden-Württemberg, Stuttgart, Theiss, p. 257-260.

1993 : " Die alamannische Landnahme im Breisgau, in: Ausgewählte Probleme europäischer Landnahmen des Frühund Hochmittelalters ", Vorträge und Forschungen, XII, p. 59-82.

\section{FORT B.}

2000 : I.e Matériel métallique du camp de BiesheimKunheim, mémoire de mâtrise, L'niversité de Dijon, inédit.

\section{FRANK K.-S., STIKA H.-P.}

1988 : "Bearbeitung der makroskopischen Pflanzen und einiger Tierreste des Römerkastells Sablonetum (Ellingen bei Weissenburg in Bavern) ", Bayerisches I.andesamt für Denkmalpflege, 61, p. 1-101.
FUCHS M. (DIR.)

1996 : Horbourg-Wihr à la lumière de l'archóologie: histoire et nouveeautés. Mélanges offerts a Charles Bonnet, Horbourg-Wihr, Association d'archéologie et d'histoire, Actes 2, 222 p.

\section{FURGER A., DESCHLER-ERB S.}

1992 : Fundmaterial aus der Schichtenfolge beim Augster Theater, Augst, Amt für Museen und Archäologie des Kantons Basel-Land (coll. Forschungen in Augst, 15), $481 \mathrm{p}$.

\section{GADOURY V., DROULERS F.}

1986 : Les Monnaies royales francaises de Louis XIII à Louis XVI (1610-1792) : cuivre, billon, argent, or, Monte-Carlo, Gadourv, $6.54 \mathrm{p}$.

\section{GARBSCH J.}

1970 : Der spätrömische Domau-Iller-Rhem-Limes, Stuttgart, Gesellschaft für Vor- und Frühgeschichte in Württemberg (coll. Kleine Schriften zur Besetzungsgeschichte Südwestdeutschlands, 6), 38 p.

1988 : “ ̈̈bersicht über den spätrömischen Donau-Iller-Rhein-Limes ", in GARBSCH J., KOS P. (ÉIS), Das spätrömische Kastell Vemania bei Isny 1. Veröffentlichungen der Kommission zur arch. Erforschung des spätrömischen Raetien, Münich, (.. H. Beck (coll. Münchner Beiträge zur Vor- und Frühgeschichte, 44), p. 105-127.

\section{Ginella F.}

2002 : Archäozoologische Auswertung von Tïerknochen spätest-römischer Befunde aus Biesheim-Kunheim/Oedenburg, Cirabung "Oedenburg-Westergass 1999" (Dép. HautRhin, Fr.) und aus Strasbourg, Crabung "Grenier d'Abondance 1999/2000" (IDép). Bas-Rhin, Fr), Diplomarbeit, Philosophisch-Naturwissenschaftliche Fakultät, Universität Basel, inćdit, 2 vol., 105 p. et $108 \mathrm{pl}$.

Ginella F, Heigold-StadelmanN A. OHNSORG P., SCHIBLER J.

2000 : “Fin Beitrag zur Nahrungswirtschaft und zur Verpflegung römischer Truppen im Legionslager Vindonissa/Windisch (CH). Archäozologische Auswertung der Tierknochen aus der Grabung Vindonissa-Feuerwehrmagazin 1976 ", Jahresbericht der (iesellschaft Pro Vindonissa, 1999 , p. 3-26.

\section{GOETHERT K.-P.}

1999: "Contionacum. Kaiserlicher I andsit\% oder vorübergehender Aufenthalt Valentinians ", Trierer Zeitschrift, 65. p. $219-221$.

\section{GOLBÉRY P. DF}

1828: Antiquités de lAlsace ou châtertux, églises et autres monuments des départements du Haut-et du Bas-Rhin, Mulhouse, Paris, Engelmann et Cie.

\section{GÖLDNER H., KREUZ A.}

1999 : "Bcgraben an einem stillen Ort: Botanische und archäologische Funde als neue Hinweise zum Leben im römische Dieburg ", Denkmalpflege und Kulturgeschichte, 2, p. 10-17.

GOSE E.

1992 : Der gallo-römische Tempelbezirk im Altbachtal zu Trier, Mainz, Philipp von Zabern (coll. Trierer Grabungen und Forschungen, 7 ), 277 p., 398 fig.

\section{GRÄDEL E.}

1989 : "Die Tierknochenfunde aus dem Amphitheater von Augusta Rauricorum (Grabungen 1982-1986) ", Jahresbericht aus dugst und Kaiseraugst, 10, p. 143-177.

\section{GRENIER A.}

1934 : Manuel d'arhéologie gallo-romaine -IIL.Archéologie du sol : les routes, Paris, Picard.

\section{HAGENDORN A}

1998 : "Neues \#um Lagerzentrum ron Vindonissa-Ausgrabungen in der Breite 1996-1998 ", Jahresbericht der (jespllschaft Pro Vindonissa, 1998, p. 23-36.

Hagendorn A., Doppler H. W., Huber A., HÜSTF.R PI.OGMANN H., JACOMET S., MEYER-FREUler C., PFÄFFLI B., SCHIBLER J.

2003: Zur Frühzeit von Vindonissa, A uswertung der Holzbauten der Grabung Windisch-Breile 1996-1998, Baden, Gesellschaft Pro Vindonissa (coll. Veröfentlichungen der (iesellschaft Pro Vindonissa, 18). $734 \mathrm{p}$.

\section{HASELIER G.}

1969: Geschichte der Stadt Breisach am Rhein -1Halbband, Von den Anfängen bis zum Jahr 1700, Breisach am Rhein, Stadt Breisach am Rhein, $478 \mathrm{p}$.

\section{HEDINGER B., LEUZINGER U.}

2002 : Tabula rasa: Holzgegenslände aus den römischen Siedlungen Vitudurum und Tasgetium, Frauenfeld, Huber, $126 \mathrm{p}$.

\section{HEGI G.}

1958 : Illustrierte Flora zon Mitteleuropa. Münich. Carl Hanser Verlag, 124 p.

\section{HEINEN H.}

1987 : "Der römische Westen und die Prätorianerpräfektur Galliens. Historischer Raum und politische Entwicklung ", Trierer Historische Forschungen, 12, p. 39-62. 


\section{HOEPER M.}

2003 : Völkerwanderungszeitliche Höhenstationen am Oberrhein. Geißkopf bei Berghaupten und Kügeleskopf bei Ortenberg, Archäologie und Geschichte, Ostfildern, Thorbecke (coll. Freiburger Forschungen zum ersten Jahrtausend in Südwestdeutschland, 12), $268 \mathrm{p}$.

\section{HOEPER M., STEUER $\mathrm{H}$.}

1999 : "Fine völkerwanderungsacitliche Höhenstation am Oberrhein- der Geißkopf bei Berghaupten, Ortenaukreis. Höhensiedlung, Kultplat\% oder Militärlager? ", Germania, 77, 1, p. 185246.

\section{HOXHA G.}

2001 : "Philon von Byzanz und die spätantiken Befestigungen in Albanien ", Archäologisches Korrespondenzblatt, 31, 3, p. 601-616.

\section{JACOMET S.}

2003 : " Lnd zum Dessert Granatapfellergebnisse der archäobotanischen Untersuchungen ", in HAGBNDORN A., DOPPLER H. W., HUBFR A., HÜSTER PIOGMANN H., JACOMET S., MFYER-

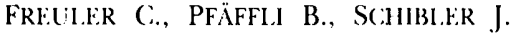
(ÉDS), Zur Frühzeit von Vindonissa, Auszertung der Holzbauten der Cirabung Windisch-Breile 1996-1998, Baden, Gesellschaft Pro Vindonisia (coll. Veröfentlichungen der Gesellschaft Pro Vindonissa, 18), p. 173-229.

\section{JACOMET S., DicK M.}

1986 : "Verkohlte Pflanzenreste aus einem römischen Grabmonument beim Augster Osttor (1966) ", Jahresberichte aus Augst und Kaiseraugst, 6, p. 7-53.

\section{JACOMET S., ERNY-RODMANN T.}

2000 : " Das Geheimnis der Flaschenkürbisse ", Basler Zeitung, 192, p. 51-53.

JACOMET S., JacQuat C., MaISE C., SCHIBLER J., STOPP B., STUdeR J., WICK L., WINTER M.

1999 : " Klima, Umwelt, Landwirtschaft und F.rnährung ", in Müll.tik F., KAlNII, (;., LüsCHER (;. (E.DS), Die Schueiz vom Paläolithikum bis zum frühen Mittelalter IV: Eïsenzeit, Basel, SGUF, p. 93-136.

\section{JACOMET S., KUCAN D., RITTER A., SUTER G.,} HAGENDORN A.

2002 : "Pomegranates (Punica granatum L.) from Early Roman Contexts in Vindonissa ", Vegotation History and Archaeobotany, 11 (1-2), p. 79-92.

\section{JACOMET S., PETRUCCI-BAVAUd M.}

2003 : «Archäobotanische Lntersuchung der Kulturschichten der Holzbauperiode ", in ScIIWARZ P.-A. (Él).), Kastelen 1. Die prähistorischen Siedlungsreste und die frühkaiserzeitlichen Holzbaulen auf dem Kastelenplateau, Augst, Amt für Museen und Archäologic: des Kantons Basel-Land (coll. Forschungen in Augst, 21), p. 241-300.

A paraître: "Samen und Früchte ", in SCHCCANY C. (E.D.), Die römische Villa von Biberist/SO (Grabungen 1982, 1983; 19861989), Untersuchungen im Wirtschajtsteil und Überlegungen zum Umland.

JACOMET S., WAGNER C., FEIGENWINTER K., FELICE N., ALBRECHT H.

1993 : Samen und Früchte aus vorrömischen, römerzeiltlichen und mittelalterlichen Ablagerungen in der Altstudt von Solothurn (Schweiz), Areale Vigier und Klosterplatz, manuscrit inédit.

\section{JACQUAT C.}

1986 : Römerzeitliche Pflanzenfunde aus Oberwinterthur (Kanton Zürich, Schweiz), Zürich, Berichte der Zürcher Denkmalpflege (coll. Archäologische Monographien, 2), p. 241-264.

JAUCH V. (ÉD.)

1997 : " Eschenz-Tasgetium: Römische Abwasserkanäle und Latrinen ", Archäologie im Thurgau, 5, $255 \mathrm{p}$.

\section{KeLler E.}

1971 : Die spätrömischen Grabfunde in Süalbayern. Veröffentlichungen der Kommission zur archäologischen Erforschung des spätrömischen Raetien, Münich, C. H. Beck (coll. Münchner Beiträge zur Vor- und Frühgeschichte, 8), $270 \mathrm{p}$.

\section{KERN E.}

1994 : "Biesheim-Kunheim (Haut-Rhin) ", in Pl:TTT J.-P., MAN(GIN M. (DIR.), Atlas des agglomerations secondaires de la (ianule Belgique et des Germanies, Paris, éd. Frrance, p. 159-161.

\section{KLEE M., JACOMET S.}

2003 : " Ergebnisse der archäobotanischen Untersuchungen ", in HüsTER Pigogmane H., Jacomet S., Ki.fe M.,

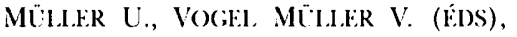
"Ein stillen Örtchen. Zur Latrinengrube in Feld 6, Grabung TOP Haus AG; Kaiseraugst (2001.01) ", Jahresberichte aus Augst und Kaiseraugst, 24, p. 178-190.

\section{KNÖRZER K.-H.}

1970 : Römerzeitliche Pflanzenfunde aus Neuss, Novaesium $I V$, Berlin, Mann (coll. Limesforschungen, 10), $161 \mathrm{p}$.

1981 : Römerzeitliche Pflanzenfunde aus Xanten,
Köln, Rheinland-Verlag, Bonn, Habelt (coll. Archäo-Physika, 11), 176 p.

\section{KNÖRZER K.H., MEURERS-BALKE J.}

1990 : « Die Wirtschafts- und Nutzungsflächen eines romischen Gutshofes. Eine Rekonstruktion aufgrund des botanischen Befundes ", in HELLENKEMPER H., HOR.N H. G., KOSCHIK H. (ÉDS), Archäologie in Nordrhein-Westfalen. Geschichte im Herzen Europas, p. 242-246.

\section{KOKABI M.}

1987 : " Tierknochenfunde aus dem Wagbachkastell bei Wiesental, Gde. Waghäusel, Kreis Karslruhe ", Fundberichte aus Baden-Württemberg, 12, p. 397-400.

\section{KOOISTRA L., HESSING W.}

1988 : « Ein frühmittelalterlicher Brunnen mit einer exotischen Frucht aus Houten ", Berichten van de Rijksdienst voor het Oudheidkundig Bodemonderzoek, 38, p. $207-228$.

\section{KREUZ A.}

1994: «Bemerkungen zu archäobotanischen Untersuchungen im römischen Vicus Hanau-Kesselstadt, Salisweg ", Neues Magazin für Hanauische Geschichte, 1994, p. 4-6.

2002 : Unerwartete Pflanzenfunde aus der keltischen Saline in Bad Nauheim, in Archäobotanik in Bad Nauheim. Wetteraukreis, Hessen, Theiss, p. 66-68.

\section{KUCAN D.}

1992 : « Die Pflanzenreste aus dem römischen Militärlager Oberaden ", in KёHLBORN J. S. LET AL. (ÉDS), Das Römerlager in Oberaden III. Die Ausgrabungen im nordwestlichen Lagerbereich und weitere Baustellenuntersuchungen, Münster, Aschendorff (coll. Bodenaltertümer Westfalens, 27), p. 237-265.

\section{KUHNLE G.}

1991 : Les Fortifications romaines de l'Antiquité lardive des vallers du Rhin supérieur at du Haut Rhin, mémoire de maîtrise, Universite de Strasbourg, $115+173 \mathrm{p}$.

\section{KÜSTER H}

1995 : Poslglaziale Vegetationsgeschichte Südbayerns. Geobotanische Studien zur prähistorischen I.andschaftskunde, Berlin, Akademie Verlag, 372 p.

\section{LAUR-BELART R.}

1991 : Ciuide d'Augusta Raurica, 5" éd. augmentée et revue par $\mathrm{L}$. Berger, Bâle, Römermuseum, 215 p., 212 fig.

LEHMANN P., BREUER G.

1997 : " The Use-Specific and Social- 
Topographical Differences in the Composition of Animal Species found in the Roman City of Augusta Raurica (Switzerland) ", in VIGiN. J.-I). (DIR.), "L'Homme et l'animal, Proceedings of the $7^{\text {th }}$ confercnce, Konstan\% 26 sept.$1^{\text {er }}$ oct. 1994 ", Anthropozoologica, 25-26, p. $487-494$.

\section{LEVI A., LEVI M.}

1967 : Itineraria picta : contributo allo studio della Tabula Peutingeriana, Rome, "L'Erma " di Bretschneider, $256 \mathrm{p}$.

\section{MACKENSEN M.}

1995 : Das spätrömische Crenzkastell Caelius Mons in Kellmünz an der Iller, Stutgart, Theiss (coll. Führer zu archäologischen Denkmälern in Bayern, 3), $118 \mathrm{p}$.

\section{MANNing W. H., ScotT R.}

1979 : "Roman Timber Military Gateways in Britain and on the German Frontier", Britannia, 10, p. 19-61.

MARINVAl P., MaRÉChal D., LABadie D.

2002 : "Arbres fruitiers et cultures jardinées gallo-romains à Longueil-Sainte-Maric (Oise) ", Gallia, 59, p. 253-271.

\section{MARTIN M.}

1991 : Das spätrömisch-frühmittelalterliche Gräberfeld von Kaiseraugst, Kı. Aargau, Derendingen, Habegger (coll. Basler Beiträge zur Ur- und Frühgeschichte, 5), $102 \mathrm{p}$

\section{MARTIN P. DE}

1989 : "Cet été de 1304 où l'on traversa le Rhin à pied en Haute-Alsace ", Physio-Géo, 19, p. 101-110.

\section{MARTINI R.}

2003 : Collezione Pangerl. Contromarche Imperiali Romane (Augustus-Vespasianus), Nomismata, Milan, Ennere (coll. Historisch-numismatische Forschungen, 6), $287 \mathrm{p}$.

\section{MAURER A.}

1993 : "Nouvelles découvertes sur le vicus d'Oedenburg-Biesheim ", Annuaire de la Société d'histoire de la Hardt et du Ried, 6, p. $8-10$.

\section{MCNicoll A. W.}

1997: Hellenistic Fortifications from the Aegean to the Fuphrates, Oxford, Clarendon Press (coll. Oxford Monographs on Classical Archaeology), $230 \mathrm{p}$.

\section{MOREL M.}

1789 : "Lettre retranscrite par J.-J. Oberlin", Almanach d'Alsare, p. 295-296.

\section{MOREL P.}

1991 : "Die osteologischen und anthropologischen Lntersuchungen ", in ETTER H. F., FEILLANA BROGLI R., FELLAMAN R., MARTIN-KHLCHER S., MOREI. P., RAST A. (ÉDS), Beiträge zum römischen Oberwinterthur-Vitudurum 5 , Zürich, Berichte der Zürcher Denkmalpflege (coll. Archäologische Monographien, 10), p. 79-185.

1994 : «Die Tierknochenfunde aus dem Vicus und den Kastellen ", in HÄNG(GI R., DOSWAL.d (., ROTH-RL'BI K. (ÉDS), Die frühen römischen Kastelle und der KastellVicus von Tenedo-Zurzach, Baden, Gesellschaft Pro Vindonissa (coll. Veröffentlichungen der Gesellschaft Pro Vindonissa, 11), p. 395-410.

\section{NIERHAUS $\mathbf{R}$.}

1940 : "Grabungen in dem spätrömischen Kastell auf dem Münsterberg von Breisach 1938 (Kreis Freiburg im Breisgau) ", Germania, 24, p. 37-46.

\section{NUBER H. U.}

2000a : "Horburg ", RGA, 15, Berlin, New York, De Gruyter, p. 113-115.

2000b: "Ein Leugensteinfragment des Postumus aus Oedenburg (Biesheim)", Annuaire de la Societé d'histoire de la Hardt et du Ried, 13, p. 15-18.

200la: "Le dispostif militaire du Rhin supéricur pendant l'Antiquité tardive et la fortification d'Oedenburg ", in Plocis S. (DIR.), La Frontiere romaine sur le Rhin supperieur. $\dot{A}$ propos de fouilles récentes de Biesheim-Kunheim, Catalogue d'exposition, Biesheim, Musée gallo-romain, p. 37-40. 200lb: " La fortication romaine tardive d'Altkirch ", in PLotin S. (DIR.), La Frontière romaine sur le Rhin supérieur. $\dot{A}$ propos de fouilles récentes de BiesheimKunheim, Catalogue d'exposition, Biesheim, Musée gallo-romain, p. 55-59. 2003 : "Spätrömische Festungen am Oberrhein ", Freiburger Lniversitätsblätter, 159, p. 93-107.

NUBER H. U., REDDÉ M. avec les contrib. de JaCOMET S., JOLY M., POPOVITCH L., SCHIBLER J., SEITZ G. et la collab. de Fort B., Matter G., Pellissier J., PETIT C., Viroulet B., WOlf J.J.

2002: "Das römische Oedenburg (Biesheim/ Kunheim, Haut-Rhin, France). Le site romain d'Oedenburg (Biesheim/ Kunheim, Haut-Rhin, France). Frühe Militärlager, Strassensicdlung und Valentinianische Festung. Les camps militaires précoces, le vicus et la forteresse de Valentinien ", Germania, 80, 1, p. 169-242.
NUBER H. U., SEITZ G.

2001 : "Frankfurts römischer Crsprung. Kastell oder Practorium ?", in HANSEN S.. PINGEL V. (ÉDS), Archäologie in Hessen. Neue Funde und Befunde, Festschrift für F:-R. Hermann zum 65. (iplurtstag, Rahden/ Westfalen, Maric Leidorf, p. 187-197.

\section{OLDENSTEIN J.}

1986 : « Neue Forschungen im spätrömischen Kastell von Alzey. Vorbericht über die Ausgrabungen 1981-1985 ", Bericht der römisch-germanischen Kommission, 67 , p. 289-356.

1994 : « Die letzten Jahrzchnte des römischen Limes zwischen Andernach und Sel\% unter besonderer Berücksichtigung des Kastells Alzey und der Notitia Dignitatum. Zur Kontinuität zwischen Antike und Mittelalter am Oberrhein ", Oberrheinische Studien, 11, p. 69-112.

OSWALD F., SCHAEFER L., SENNHAUSER H. R. 1966 : Vorromanische Kirchenbaulen, Kalalog der Denkmäler bis zum Ausgang der Ollonen, Münich, Prestel, $432 \mathrm{p}$.

OXÉ A., COMFORT H.

1968: Corpus Vasorum Arretinorum, vol. 4, Bonn, Habelt (coll. Antiquitas Reihe, 3), 616 p.

Pals J. P., HakBijl T.

1992 : "Weed and Insect Infestation of a Grain Cargo in a Ship at the Roman Fort of Laurium in Woerden (Province of Zuid-Holland) ", Review of Palaeobotamy and Palynology, 73, p. 287-300.

PASSARD F., URLACHER J.P. avec les contrib. de Avanzi V., Chenevov M.-H., LAVIER C., LAMBERT G., LUNDSTRÖMBAUDAIS K., RICHARD H. et avec la collab. de BAUDAIS D., FISCHER B., MANFREDICizARD S., Olive C.

1997 : «Besançon, Saint-Jean (Doubs) : structures en fosses en contextes laténien et gallo-romain ( $\mathrm{I}^{\text {er }}$ siècle avant J-C. $\mathrm{I}^{\mathrm{er}}$ siècle après J.-(.) ", Reoue archéologique de l'Est, 48 , p. 167-218.

\section{PETRIKovits H. VON}

1975 : Die Innenbaulen römischer Legionslager während der Prinzipatszeit, Opladen, Westdeutscher Verlag (coll. Abhandlungen der RheinischWestfälischen Akademie der Wissenschaften, 56), 227 p.

\section{PeTRuCCI-BAVAud M.}

1996: "Pflanzliche Speisebeigaben in den Brandgräbern ", in HAFFFIIĹ (. (ÉD).), "Die römischen Gräber an der Rheinstrasse 46 des Nordwestgräberfeldes von Augusta Raurica ", Jahresberichte aus Augst und Kaiseraugst, 17, p. 253-259. 
Petrucci-Bavaud M., Jacomet S. avec la collab. de NARTEN G. B.

2000 : " Samen, Früchte und Fertigprodukte ", in HINTERMAN D. (E.D.), Der Südfriedhof von Vindonissa, Brugg (coll. Veröffentlichungen der Gesellschaft Pro Vindonissa, 17) p. 151-159.

\section{PétRY F.}

1982a: "Biesheim, 2. Archéologie, (aalloromain ", in ACKF A. (DIR.), Encyclopédie de l'Alsace, vol. 1, Strasbourg, éd. Publitotal, p. 621-624.

1982b : "Circonscription d'Alsace ", Ciallia, 40,2, p. $347-371$.

PÉTRY F., KERN E.

1978 : "Un Mithraeum à Biesheim ", Cahiers d'archéologie el d'histoire d'Alsace, 21, p. 5-32.

\section{PFÄFFLI B., SCHIBLER J.}

2003 : "Die Tierknochen aus den vorlagerund lagerzeitlichen Fundschichten aus der Grabung "Breite" im Bereich des römischen Legionslagers Vindonissa ", in HAGENDORN A., DOPPI.ER H. W. Heber A., HÉSTER PlogmanN H. JACOMET S., MEYFr-Freller C., PfäFFl. B., SCHIBI.FR J. (ÉDS), Neue Forschungen zur Frühzeit von Vindonissa und den Lagern der 13. Legion, Baden, Gesellschaft Pro Vindonissa (coll. Veröfentlichungen der Gesellschaft Pro Vindonissa, 18), p. 244-279.

PLOUIN S. (DIR.)

2001 : La lrontière romaine sur le Rhin supérieus: A propos des fouilles récentes de Biesheim Kunheim, Catalogue d'exposition, Biesheim, Musée gallo-romain, $104 \mathrm{p}$.

\section{Pollmann B.}

2003 : Archäobotanische Makrorestanalysen und molekulararchäologische Untersuchungen an botanischen Funden aus dem römischen vicus Tasgetium (Eschenz/Kanton Thurgau/(CH), Diplomarbeit, Universität Basel, 105 p.

\section{RAPPORT}

1998 : NLBER H. U., in REDDE. M. (DIR.) Oedenburg, Rapport de la campagne préliminaire menćc en 1998 à BiesheimKunheim et programme triennal 19992001 , p. 37-39.

1999 : NCBbir H. U., SEIT\% (;., " Dic Ausgrabungen in Westergass (chantier 99.02) et Altkirch (chantier 99.03) ", in REDDFF. M. (DIR.), Oedenburg, Rapport de la campagne menéc en 1999 à BiesheimKunheim et programme triennal 2000 $2002(2000)$, t. II, 2.

2000 : NLber H. U., SEITZ (.., "I .es fouilles du Westergass (chantier 02) et les fouilles
d'Altkirch (chantier 03) ", in RF.DDÉ M. (DIR.), Oedenburg 2000, Premier rapport intermédiaire du programme triennal de fouilles sur le site de Biesheim-Kunheim, Paris, p. 38-48.

2001 : NCBER H. U., SEITZ G., "I.es fouilles d'Altkirch (chantier 03) ", in REDDE. M. (DIR.), Oedenburg: fouilles 2001, Rapport intermédiaire $\mathrm{n}^{\circ}$ 2, Paris, p. 99-116.

2002 : NUBER H. U., RF.DDÉ M., " L'Antiquité tardive (Altkirch et Westergass) ", in REDDÉ M. (DIR.), Rapport triennal (2000) 2002) sur les fouilles franco-germano-suisses a Oedenburg (Haut-Rhin), p. 187-250.

\section{REDDÉ M.}

À paraître: "Où sont passés les castella Drusiana? Réflexions critiques sur les débuts de l'occupation militaire romaine dans le bassin du Rhin supérieur ^, Revue d'Etudes militaires antiques, 2.

REDDÉ M., NUBER H. U.

1999 : « Les fouilles sur le site militaire romain d'Oedenburg: premiers résultats ", Annuaire de la Société d'histoire de la Hardt et du Ried, 12, p. 5-14.

Reddé M., Brulet R., Fellmann R., HAALEBos J. K. SchNURbeIN S. VoN À paraître: Manuel d'architecture gallo-romaine -I- Les Fortifications militaires, Paris, Maison des sciences de l'homme (coll. DAF).

\section{RITTERLING E.}

1913 : " Das frührömische Lager bei Hotheim im Taunus ", Annalen des Vereins für Nassauische Altertumskunde und Geschichtsforschung, 40, 1-416.

\section{RöSCH M.}

1995 : " Römische Brumnen in Lahr. Fundgruben für die Botanik ", Archäologische Ausgrabungen in BadenWïttemberg, 1994, p. 151-156.

\section{SCHIBLER J.}

À paraître: "Hinweise zur Romanisierung aufgrund archäozoologischer Ergebnisse aus der Schweiz und dem angrenzenden Ausland. Vortrag: 2. Treffen der Arbeitsgruppe zur Erforschung der spätlatène-frühkaiserzeitlichen Übergangsæeit nördlich der Alpen ", Romanisierung: militürisch oder zivil ?, Basel, 2001.

\section{SCHIBLER J., FURGER A.}

1988 : Die Tierknochenfunde aus Augusta Raurica (Crabungen 1955-1974), Augst, Römermuseum (coll. Forschungen in Augst, 9), $240 \mathrm{p}$.

\section{SCHIEK S.}

1992 : Das Gräberfeld des Merowingerzeit bei Oberflacht, Stuttgart, Theiss, $166 \mathrm{p}$., 113 fig.

\section{SCHLAEFLI L.}

1983 : "Glanes historiques sur le village disparu d'Oedenburg ", Almanach SainteOdile, p. 42-44.

\section{Schilumbaum A., Petrucci-Bavaun $M$}

S. d. : Die Pflanzenreste aus fünf römerzeitlichen Brandgräbern in Reinach (Baselland), manuscrit inédit.

\section{SCHMAEDECKE M.}

1992 : Der Breisacher Münsterberg. Topographie und Entwicklung, Stuttgart, Theiss (coll. Forschungen und Berichte der Archäologic des Mittelalters, 11), 289 p.

SCHNURBEIN S. VON, KÖHLER H.-J.

1989 : «Der neue Plan des valentinianischen Kastells Alta Ripa (Altrip) ", Bericht der Römisch-Germanischen Kommission, 70 , p. 507-526.

SCHÖPFLIN J.-D. (trad. RAVENEZ L. W.)

1849: "Autres localités habitées par les Romains en Alsace. Haut-Rhin. 595-600 ", in L'Alsace illustrée, Mulhouse, F. Perrin.

\section{SCHULTZE R.}

1909 : “ Die römischen Stadttore ", Bonner Jahrbuch, 118, p. 280-352.

1928 : Basilika. Untersuchungen zur antiken und frühmittelalterlichen Baukunst, Berlin, Leipzig, De Gruyter (coll. RömischGermanische Forschungen, 2), 87 p.

\section{SCHWEITZER J.}

1984 : « Le site d'Illzach », in SCHWETT/ER J. (DIR.), L'Habitat rural en Alsace au haut Moyen Âge, Riedsheim, J. Schweitzer, $382 \mathrm{p}$.

\section{SCHWINDEN L.}

1985 : " Römer\%citliche Bleietiketten aus Trier. Zum Handel mit Pfeffer, Arznei und Kork ", Trierer Zeitschrift, 48, $121 \mathrm{p}$.

\section{SEITZ G.}

2001 : « Le praetorium d'OedenburgWestergass ", in PLOUIN S. (IIR.), La Frontière romaine sur le Rhin supérieur: $A$ propos des fouilles récentes de BirsheimKunheim, Catalogue d'exposition, Biesheim, Muséc gallo-romain, p. 50-54.

2003 : «Villen, Vici, Bäder: dic römische Besiedlung des rechten Rheinufers, in Kelten, Römer und Germancen: frühe Gesellschaft und Herrschaft am Oberrhein bis zum Hochmittclalter ", Freiburger Universitätsblätler, 159, p. 47-63.

Sell Y., Berchtold J.-P., Callot H., HOFF M., GaLl J.-C., WALTER J.-M.

1998 : L'Alsace et les Vosges. ('íologie, milieux naturels, flore el faune, Paris, Delachaux et 
Niestlé (coll. La Bibliothèque du naturaliste), $354 \mathrm{p}$.

\section{SOMMER C. S.}

1988 : " Kastellvicus und Kastell. Untersuchungen Aum Zugmantel im Taunus und $z u$ den Kastellvici in Obergermanien und Rätien ", Fundberichte aus Baden-Württemberg, 13, p. $457-707$.

1994a : "Les agglomérations secondaires de la Germanie Transrhénanie (rechtsrheinisches Germanien) ", in PETIT J.-P., MANGIN M. (DIR.), Les Agglomérations secondaires. La Gaule Belgique, les Germanies et l'Ocrident romain, Colloque BliesbruckReinheim/Bitche, 1992, Paris, éd. Errance, p. 89-102.

1994b: " Das rechtsrheinische Obergermanien ", in PFTIT J.-P., MANGIN M. (DIR.), Allas des agglomérations secondaires de la Gaule Belgique et des Germanies, Paris, éd. Errance, p. 137-146.

1995 : " Vom Kastell zur Stadt. Aspekte des C̈bergangs in Lopodunum/Ladenburg und Arae Flaviae/Rottweil ", in GROENMAN W., VAN WAATERINGF W., VAN BEEK B. L., WILIEMS B. J. H., WYNIA S. L. (ÉDS), Roman Frontier Studies, Oxford, Oxbow Books (coll. Oxbow Monograph, 91), p. $511-516$.

1997 : « Traian und die Errichtung der Civitas Ulpia Sueborum Nicrensium ", in S(HAILMAYER E. (ÉD.), Traian in Germanien, Traian im Reich, Bad Homburg, Bericht des dritten Saalburgkolloquiums, p. 241-246.

1998 : Kastellvicus und Kastell. Modell für die Canabae Legionis?, Jahresbericht Gesellschaft Pro Vindonissa, 1997, p. 41-5̆2.

1999 : "The Roman Army in SW Germany as an Instrument of Colonisation: the Relationship of Forts to Military and Civilian Vici ", in GOL.DSWORTHY A., HAYNES I. (ÉDS), The Roman Army as a Community. Including Papers of a Conference held at Birkbeck College, University of London on 11-12 January 1997, Portsmouth, s.e., p. 81-93.

\section{SPEIDEL M. A.}

1996 : Die römischen Schreibtafeln von Vindonissa : lateinische Texte des militärischen Alltags und ihre geschichtliche Bedeutung, Brugg, Gesellschaft Pro Vindonissa, 271 p.

\section{STIKA H.-P.}

1996 : Römerzeitliche Pflanzenreste aus BadenWürttemberg, Stuttgart, Theiss (coll. Materialhefte zur Archäologie in BadenWürttemberg, 36), $207 \mathrm{p}$.

\section{SWOBODA R. M.}

1986 : Die spätrömische Befestigung Sponeck am Kaiserstuhl, Münich, C. H. Beck (coll. Münchner Beiträge zur Vor- und Frühgeschichte, 36), 205 p.

ThÉobald N., MÉnillet F., LOUGNON J., BONNET C., FLUCK P.

1978: Notice explicative de la carte géologique de la France au $1 / 50000$, Feuille Neuf-Brisach.

THORNE C. R., RUSSEl A. P. G., ALAM M. K. 1993 : "Planform Pattern and Channel Fvolution of the Bramaputra River, Bangladesh ", in BEST J. L., BRISTOW C. S. (EDS), Braided Rivers, Londres, Geological Society (coll. Geological Society, Special Publication 75), p. 257-276.

\section{UERPMANN H.-P.}

1977 : " Schlachterei-Technik und Fleischversorgung im römischen Militärlager von Dangstetten (Landkreis Waldshut) ", Regio Basiliensis, XVIII, I, p. $261-272$.

\section{VAN ANDRINGA W. (DIR.)}

2000 : Archéologie des sanctuaires en Gaule romaine, Saint-Étienne, Université de Saint-Étienne, $210 \mathrm{p}$.

\section{VOGT H.}

1988 : "Le Site de Strasbourg ", in Aux origines de l'Alsace, du Paléolithique au Mésolithique, Strasbourg, Les Musées de la ville de Strasbourg, p. 10-12.

\section{WEBER G.}

1989 : APC Archäologischer Park Cambodunum -1Abschnitt. Der gallorömische Tempelbezirk : ein Begleitheft mit den Texten und Abbildungen der Ausstellung in der inneren Doppelhalle des teilrekonstruierten Tempelbezirks, Kempten, Kulturamt, Archäologic Abteilung, 70 p., 70 fig.

\section{WEBER G. (ÉD.)}

2000 : Cambodunum-Kempten. Erste Hauptstadt der römischen Provinz Raetien?, Mainz, Philipp von Zabern, 156 p., 250 fig.

\section{WEBER-JENISCH G.}

1995 : Der Limberg bei Sasbach und die spätlatènezeitliche Besiedlung des Oberrheingebietes, Stuttgart, Theiss (coll. Materialhefte zur Archäologie in Baden-Württemberg, 29), $207 \mathrm{p}$.

\section{WESCH-KLEIN G.}

1989 : "Breisach am Rhein : die gestempelten Ziegel aus den Grabungen 1983-1986", Fundberichte aus Baden-Würtlemberg, 14. p. $387-426$.
WHEELER R. E. M., WHEELER T. V.

1932 : "Reports on the Fxcavation of the Prehistoric. Roman and PostRoman Site in Lydney Park, Gloucestershire ", Reports of the Researrh Committe of the Society of Antiquaries of London, 9, 137 p., $52 \mathrm{pl}$.

\section{WIEGELS R.}

1983 : "Zeugnisse der 21. Legion aus dem südlichen und mittleren Oberrheingebiet. Zur Geschichte des obergermanischen Heeres um die Mitte des 1. Jahrhunderts n. Chr: ", Ippigraphische Studien, 13, p. 1-42.

\section{WIETHOLD J.}

1998 : “Archäobotanische Aspekte der Romanisierung in Südwestdeutschland. Bemerkungen zur Unkrautflora römerzeitlicher Dinkeläcker, Internationale Archäologie ", Studia Honoraria (Festschrift A. Haffner), 4, p. $531-551$.

\section{WILSDORF-COLLET N.}

1998 : "Le Conseil souverain au XVII" siècle : La Villeneuve de Brisach et le milieu parlementaire ", Annuaire de la Société d'histoire et d'archéologie de Colmar, XI.III, p. 19-29.

\section{ZACH B.}

2002 : "Vegetable Offerings on the Roman Sacrificial Site in Mainz, Germany: Short Report on the First Results ", Vegetation History and Archaeobotany, 11, p. 101-106.

\section{ZAGERMANN M.}

2003 : Metallene Tracht- und Ausrüstungsgegenstände des 3. bis 5. Jahrhunderts n. Chr: aus Oedenburg (Biesheim, Haut-Rhin, Fr:), mémoire de maîtrise, Université de Fribourg-en-Brisgau, non publié.

\section{ZAHN E.}

1991 : Die Basilika in Trier: Römisches Palatium. Kirche zum Erlöser, Trier, Rheinisches Landesmuseum (coll. Schriftenreihe des Rheinischen Landesmuseums Trier, 6), $87 \mathrm{p}$.

\section{ZEHNER M.}

1998 : Le Haut-Rhin, Paris, Académie des inscriptions et belles-lettres (coll. Carte archéologique de la Gaule, 68), $375 \mathrm{p}$.

\section{ZUCCA R.}

1992 : " Un'inscrizione dall'Oristanese ", L'Africa romana, 9, p. 13-15. 